\title{
Preparing for physics at the LHC
}

Fabiola Gianotti

(CERN, PH Department)

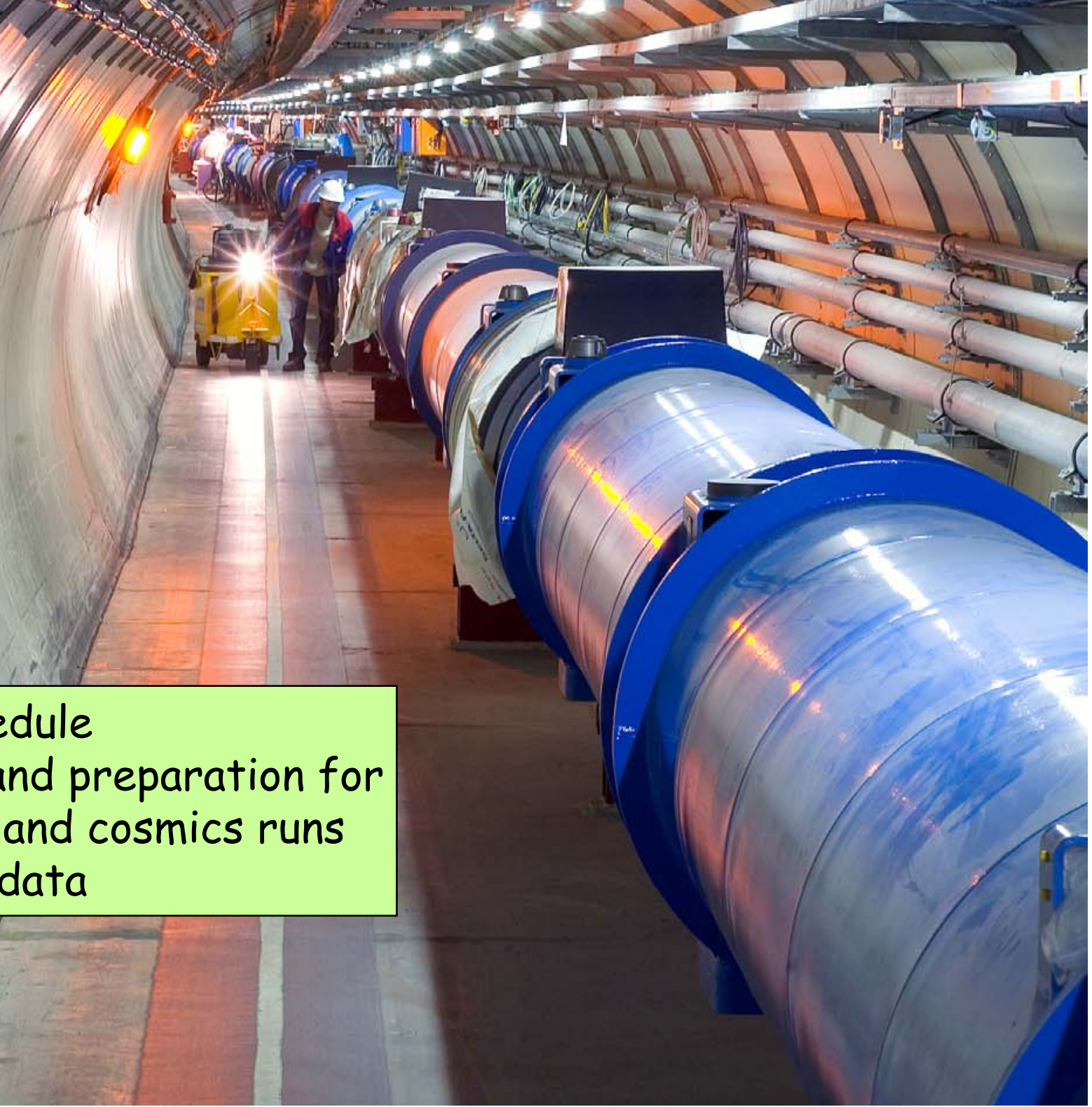

is Machine status and schedule

(1) Status of experiments and preparation for physics with test beams and cosmics runs

(1) First physics with first data 


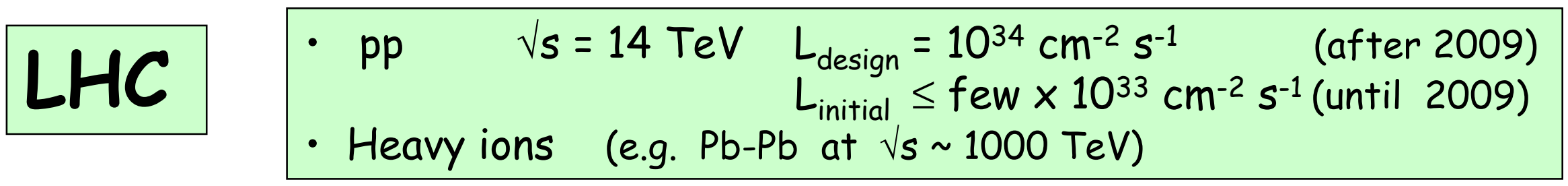

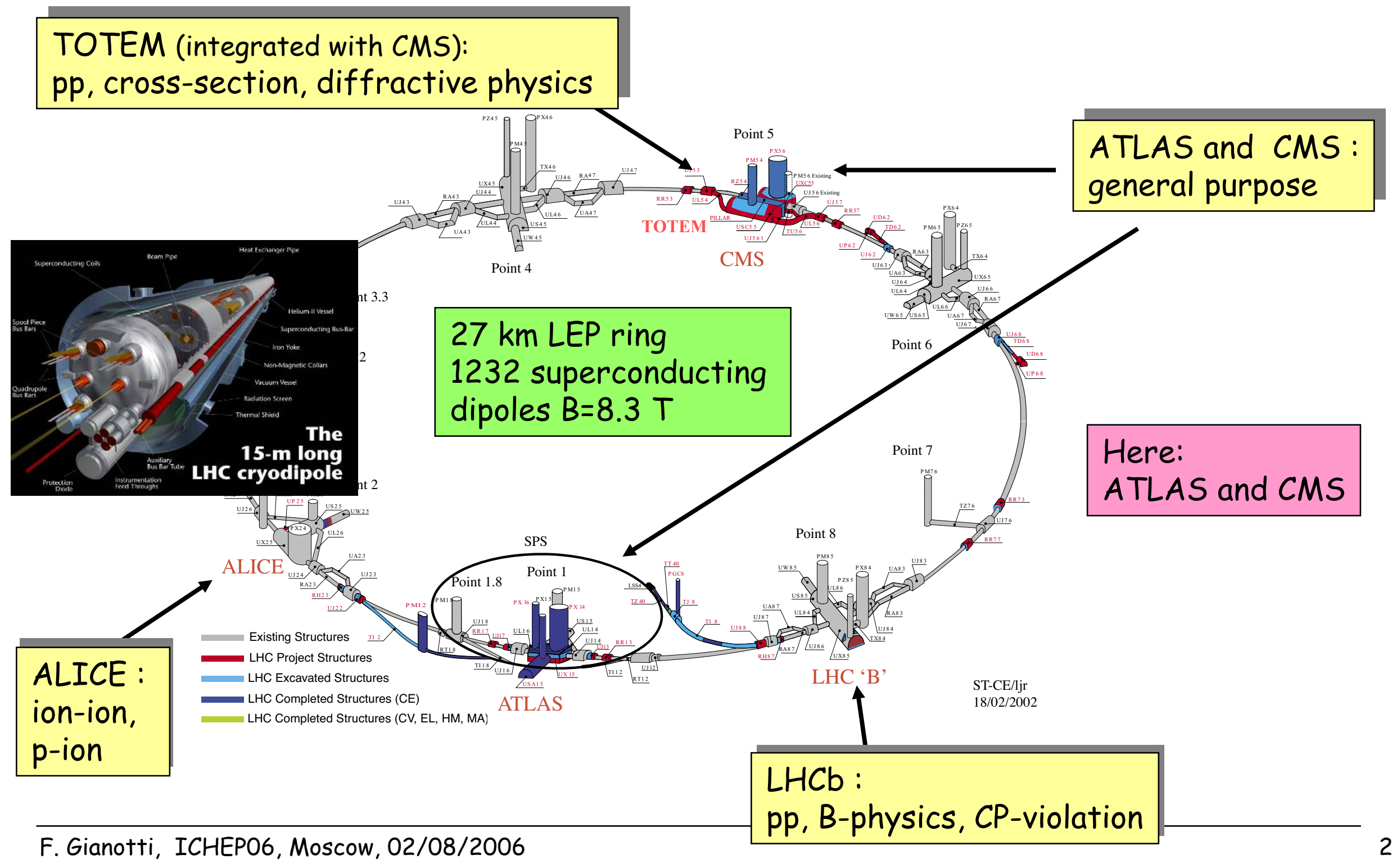




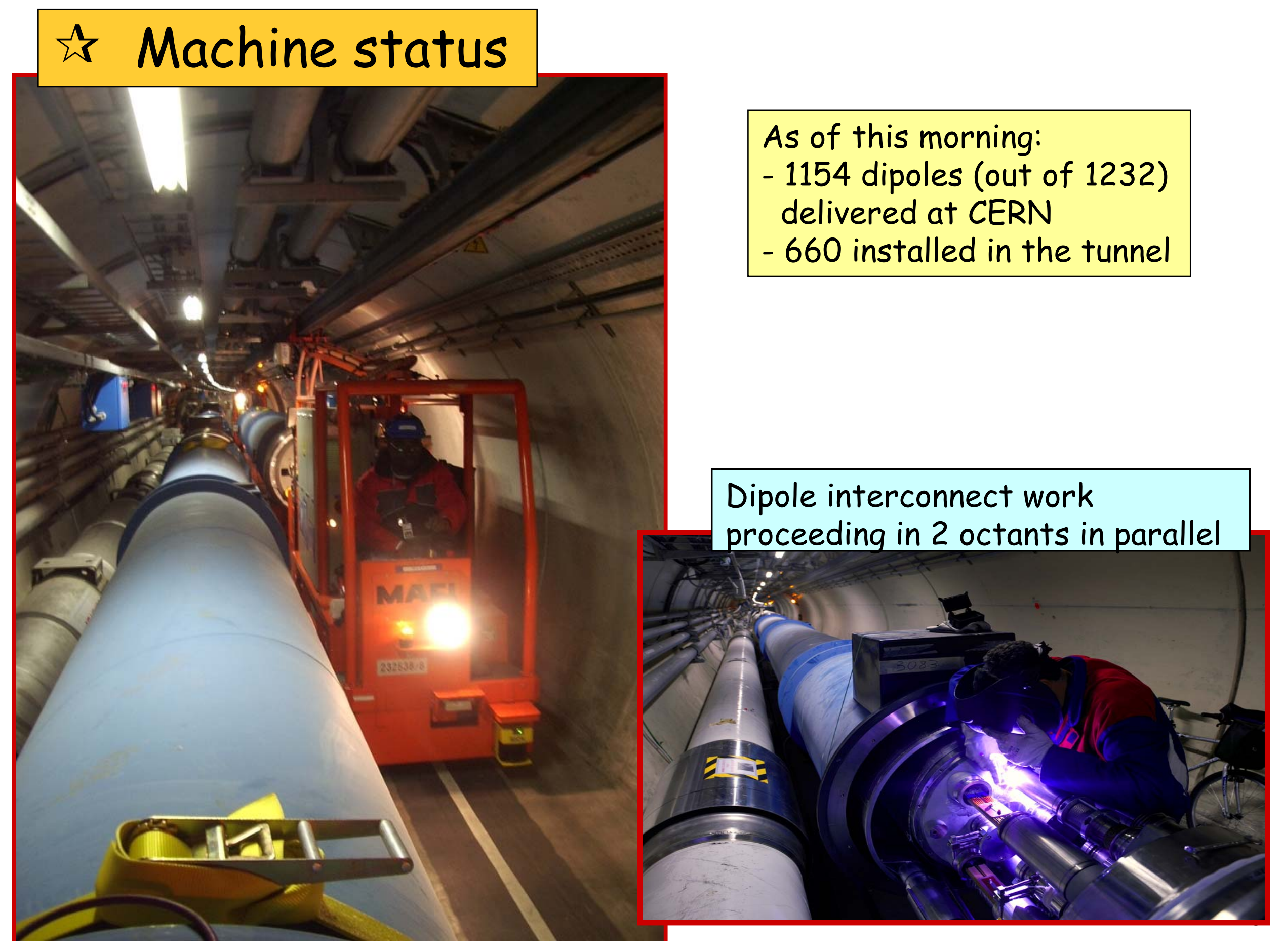


Not only dipoles....

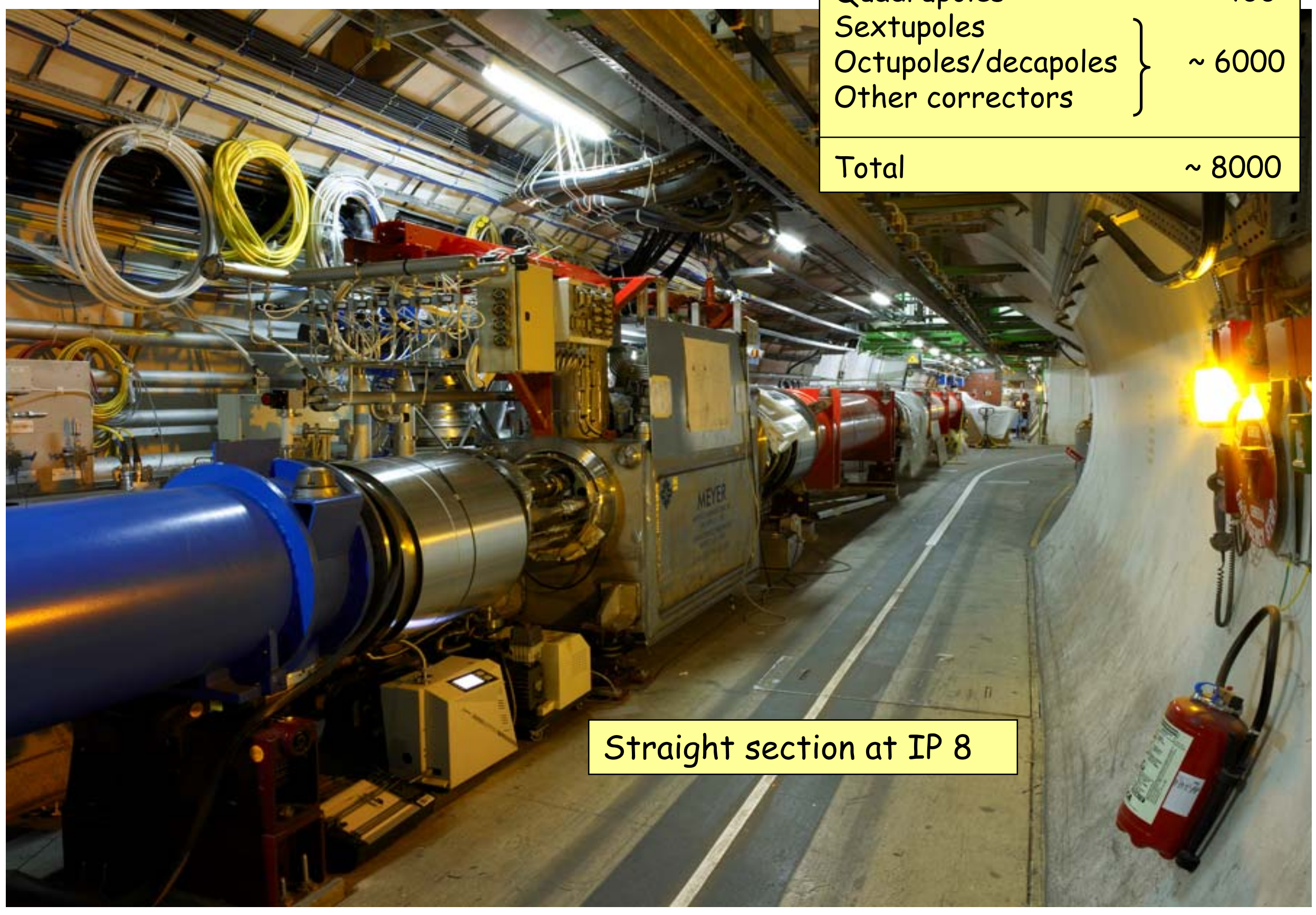

Main dipoles Quadrupoles

1232

$\sim 400$

Sextupoles

Octupoles/decapoles

Other correctors

Total

$\sim 8000$ 


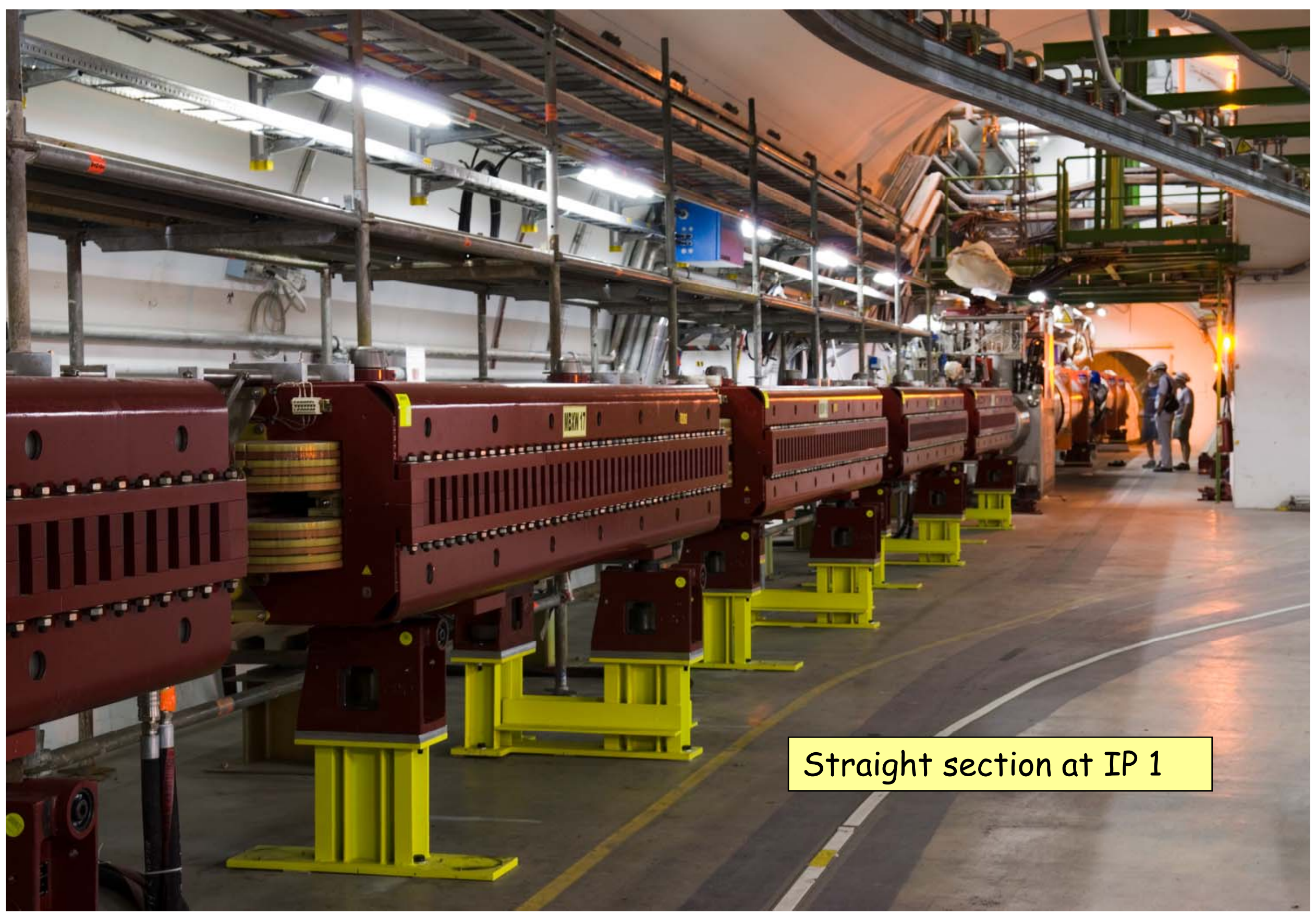

F. Gianotti, ICHEP06, Moscow, 02/08/2006 
First 600 m of cryoline (QRL) successfully cooled down on 14/9/2005, followed by cool-down of full cryoline sector 8-1 and pressure test of sector 4-5.

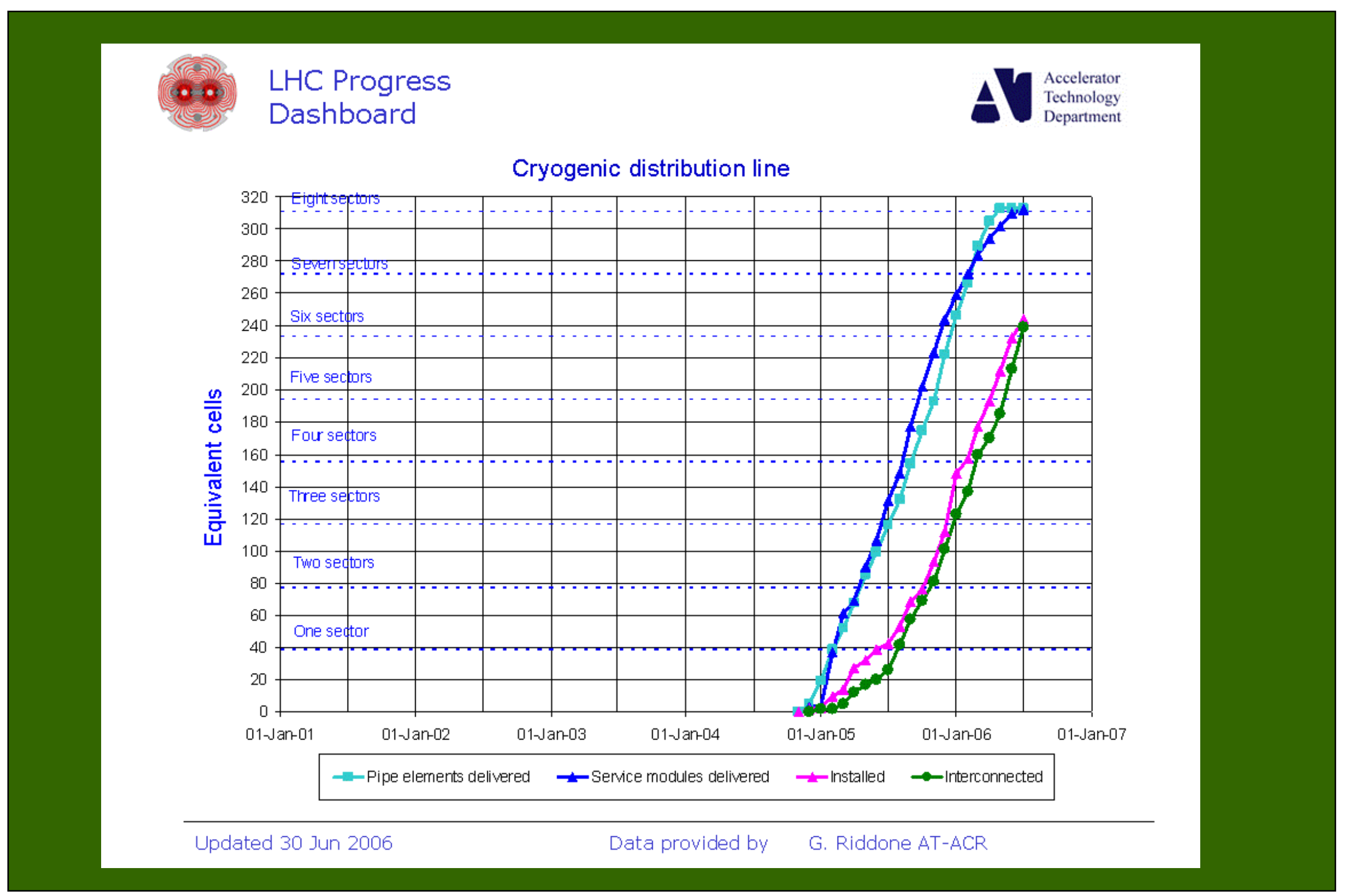




\section{(Revised) LHC schedule}

as presented to CERN Council on 23 June 2006

- Last magnet installed Machine and experiments closed
: March 2007 : 31 August 2007

- First collisions ( $\sqrt{s}=900 \mathrm{GeV}, \mathrm{L} \sim 10^{29} \mathrm{~cm}^{-2} \mathrm{~s}^{-1}$ ) : November 2007 Commissioning run at injection energy until end 2007 , then shutdown (3 months ?)

- First collisions at $\sqrt{ } s=14 \mathrm{TeV}$ (followed by first physics run): Spring 2008 Goal : deliver integrated luminosity of few $\mathrm{fb}^{-1}$ by end 2008

- Sectors 7-8 and 8-1 will be fully commissioned up to 7 TeV in 2006-2007. If we continue to commission the other sectors up to $7 \mathrm{TeV}$, we will not get circulating beam in 2007.

- The other sectors will be commissioned up to the field needed for de-Gaussing.

- Initial operation will be at $900 \mathrm{GeV}(\mathrm{CM})$ with a static machine (no ramp, no squeeze) to debug machine and detectors.

- Full commissioning up to $7 \mathrm{TeV}$ will be done in the winter 2008 shutdown

L. Evans, CERN Council, 23/6/2006 


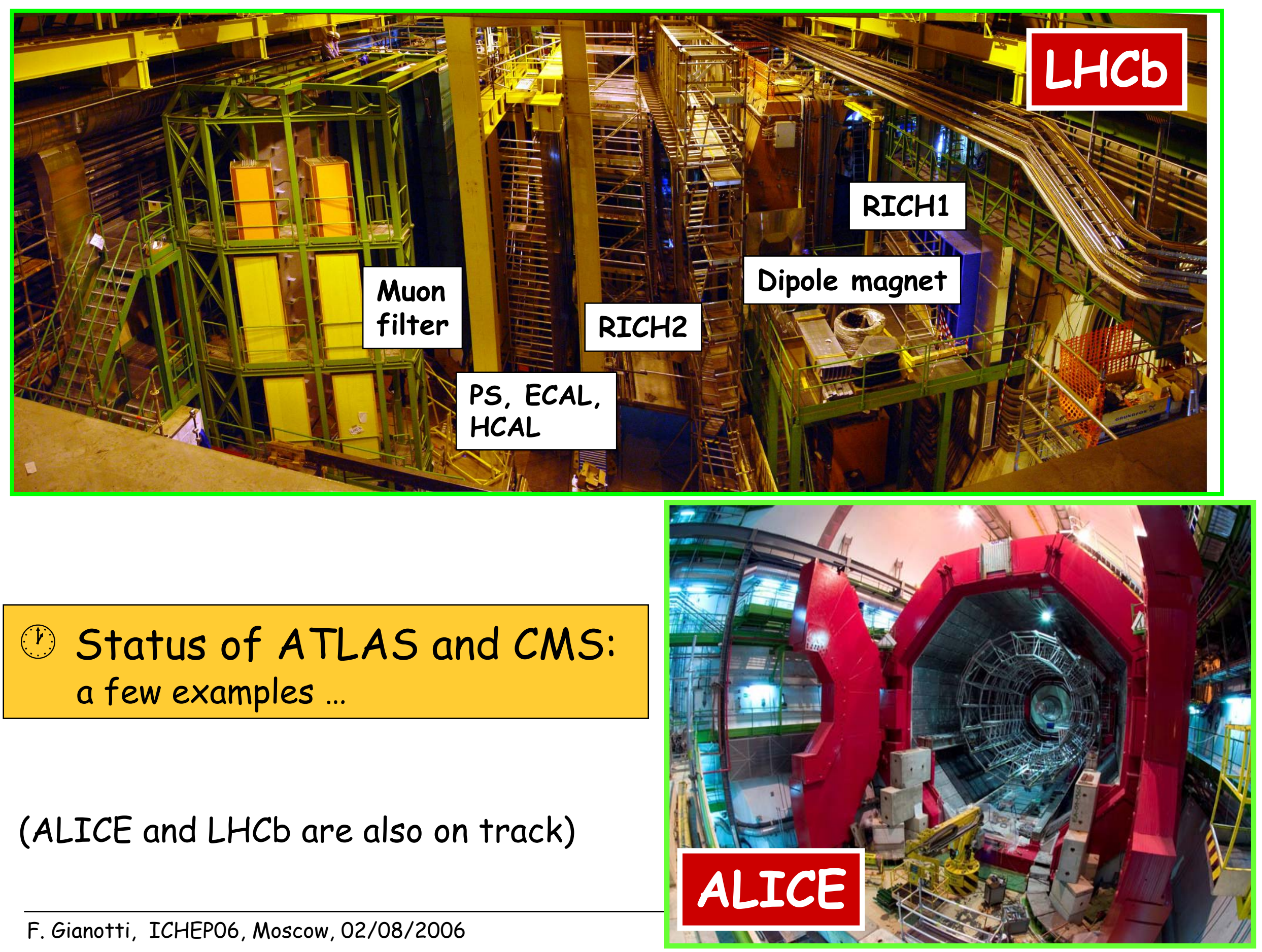




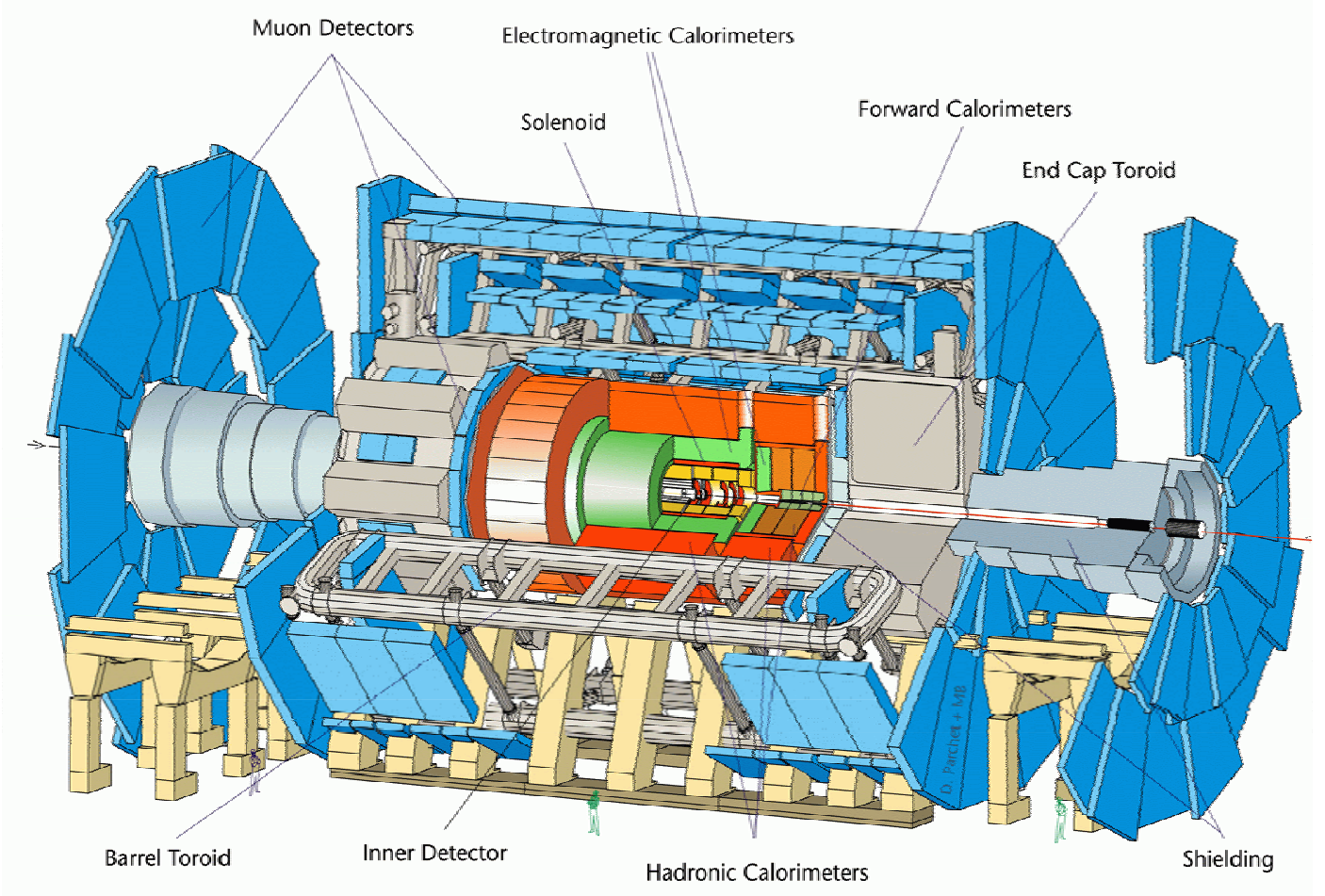

ATLAS

Length : 45 m

Radius : $\sim 12 \mathrm{~m}$

Weight : 7000 tons

Electronic channels : 108

$3000 \mathrm{~km}$ of cables
- Tracking $(|\eta|<2.5, \mathrm{~B}=2 \mathrm{~T})$ :

-- Si pixels and strips

-- Transition Radiation Detector (e/ $\pi$ separation)

- Calorimetry $(|\eta|<5)$ :

-- EM : Pb-LAr with Accordion shape

-- HAD: Fe/scintillator (central), Cu/W-LAr (fwd)

- Muon Spectrometer $(|\eta|<2.7)$ :

air-core toroids with muon chambers 


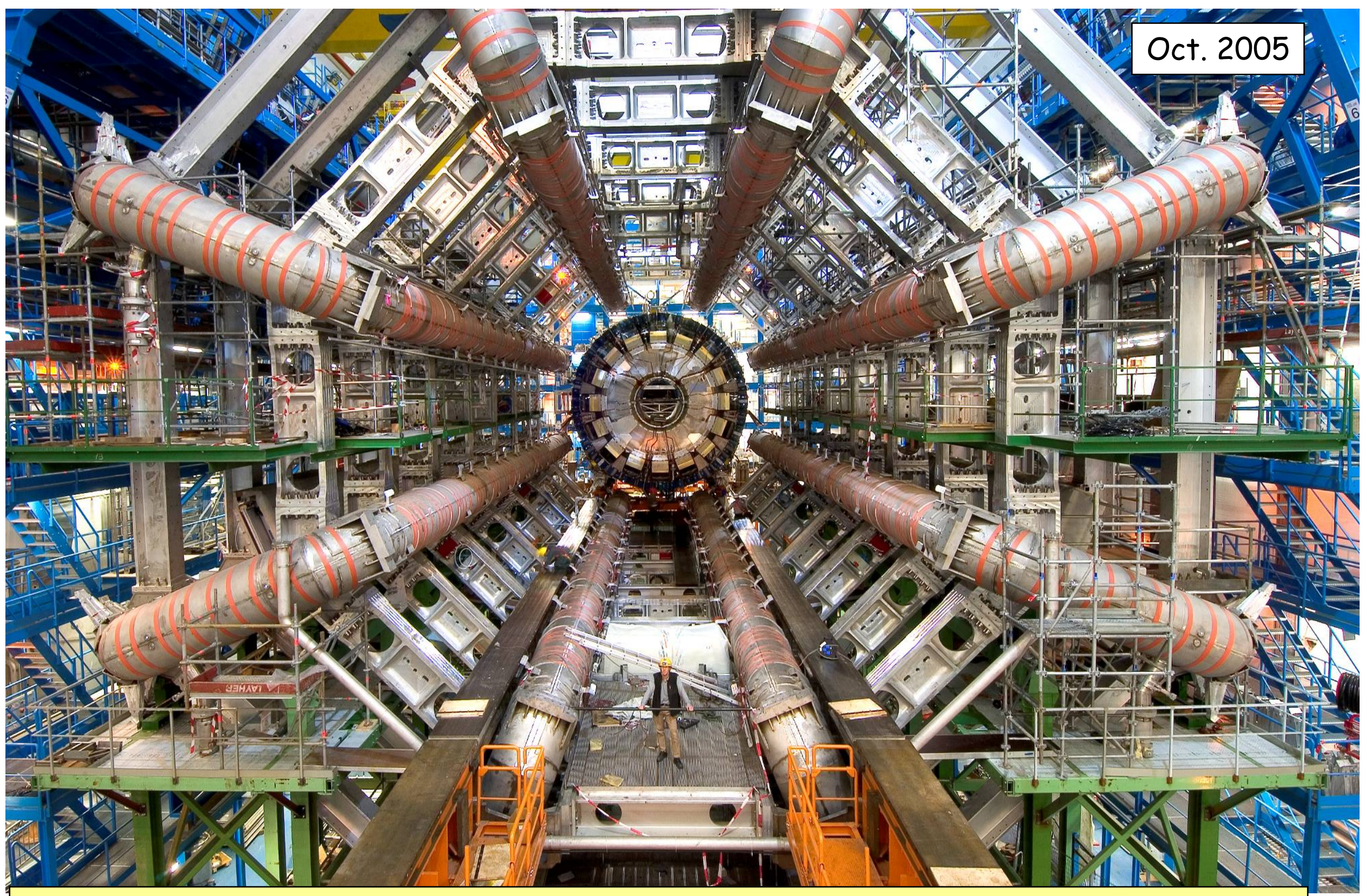

Barrel toroid: cool down started (this morning T 120 K), first tests of full field in Sept. End-cap toroids: will be installed in the pit end 2006-beg 2007 


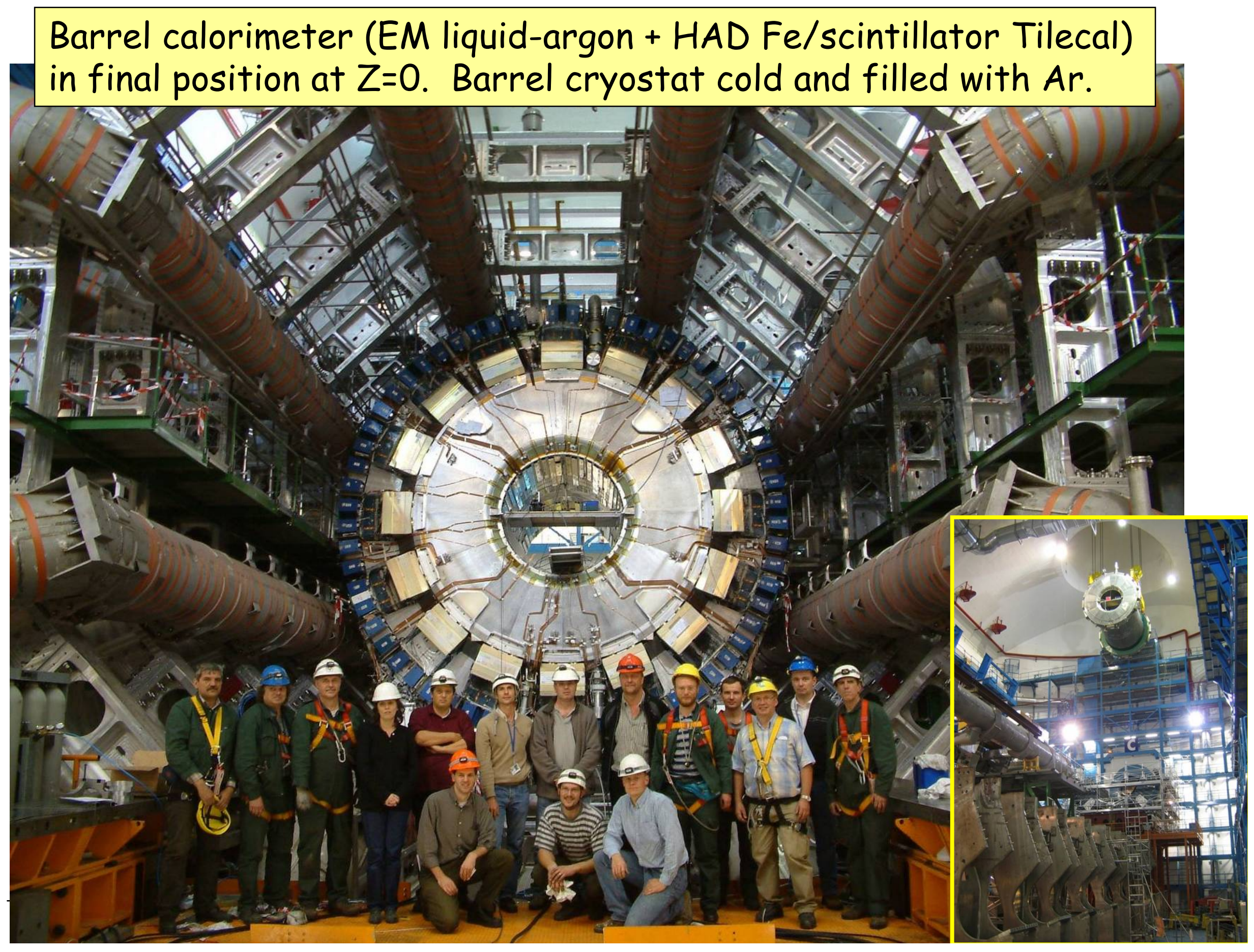


One end-cap calorimeter (LAr EM, LAr HAD, LAr Forward inside same cryostat, surrounded by HAD Fe/Scintillator Tilecal) being moved inside the barrel toroid

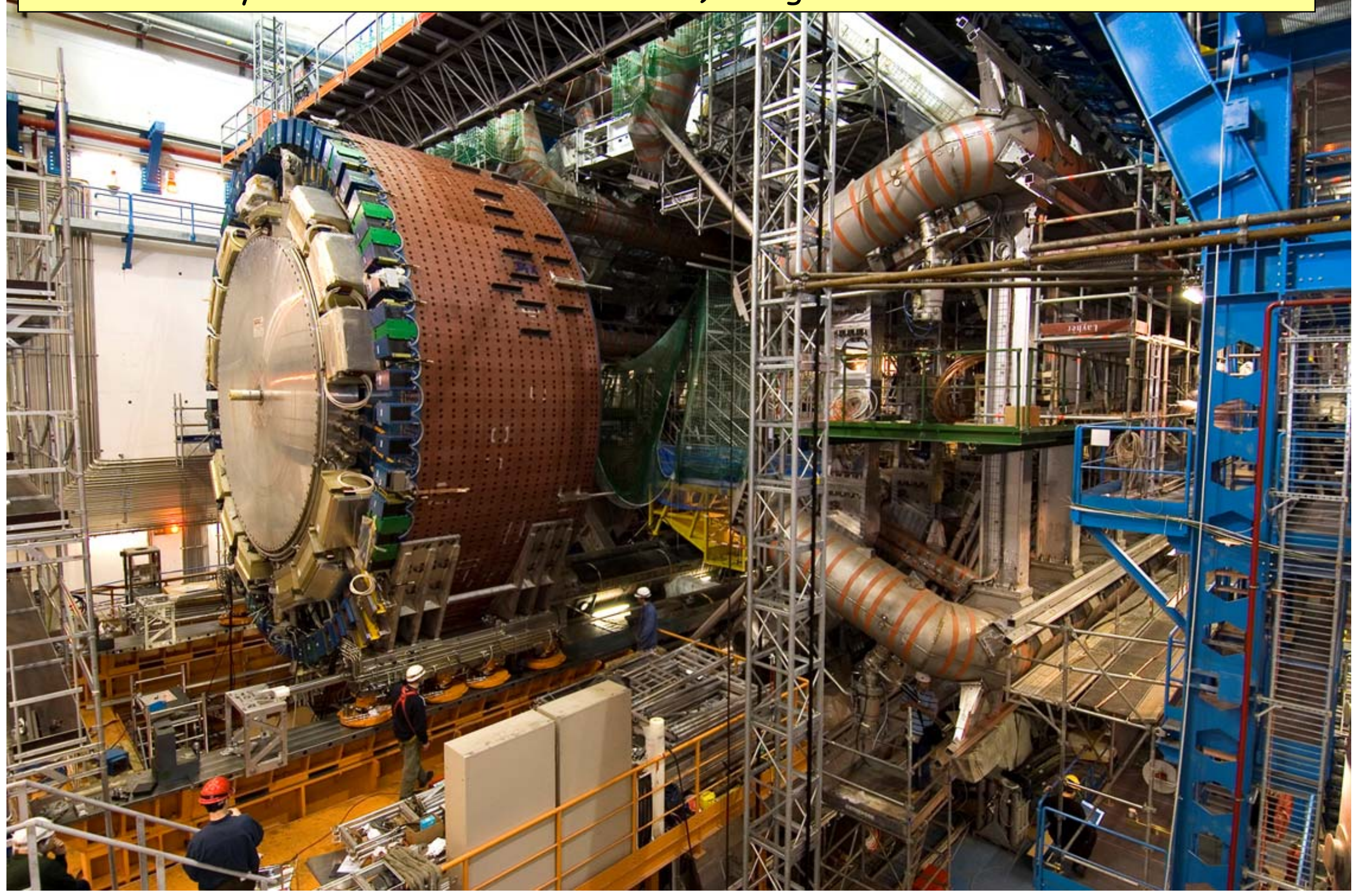




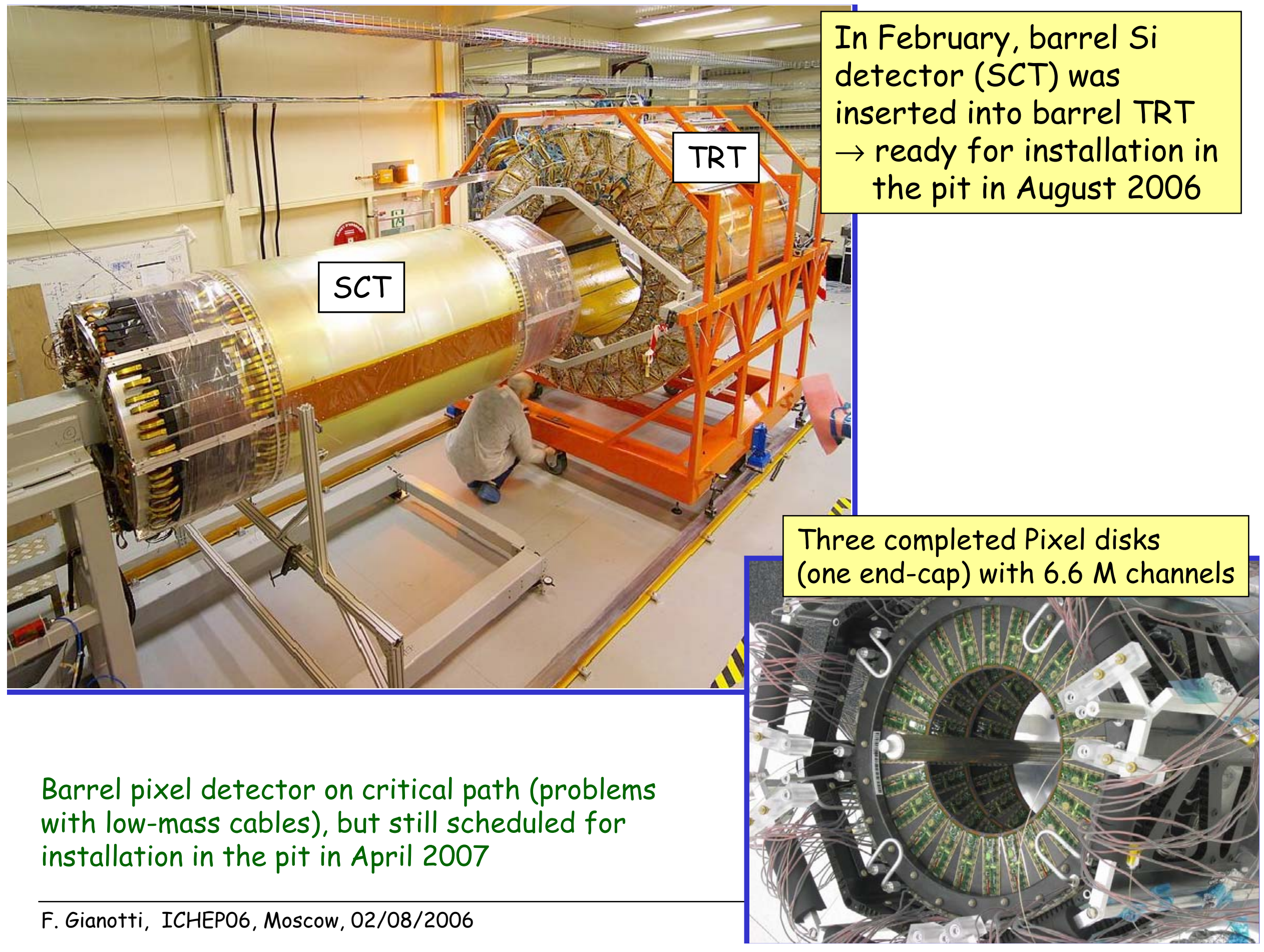




\section{Cosmics DATA taken in barrel SCT+TRT : 450k events}

QuickTime $^{\mathrm{TM}}$ and a are needed to see this picture.

QuickTime $^{\mathrm{TM}}$ and a TIFF (LZW) decompressor are needed to see this picture

Cabling error in two modules
ATLAS preliminary

\section{SCT efficiency} per layer

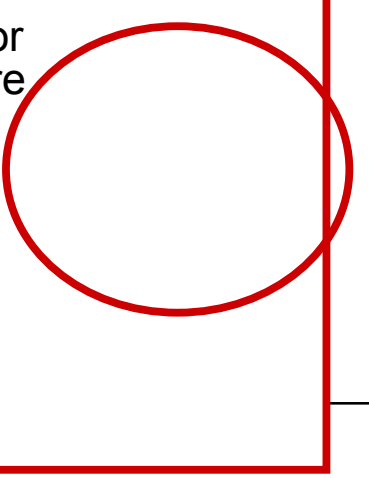

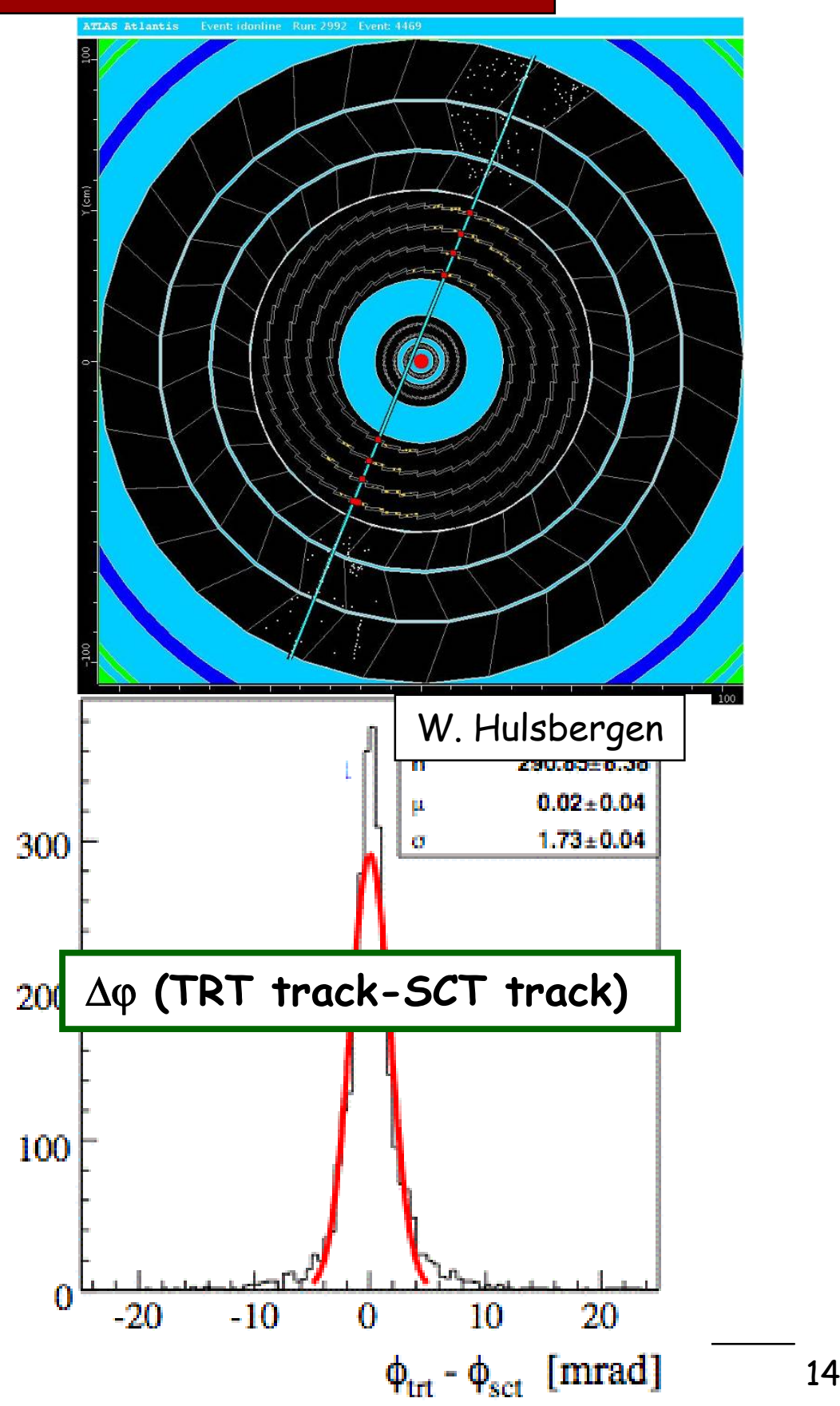




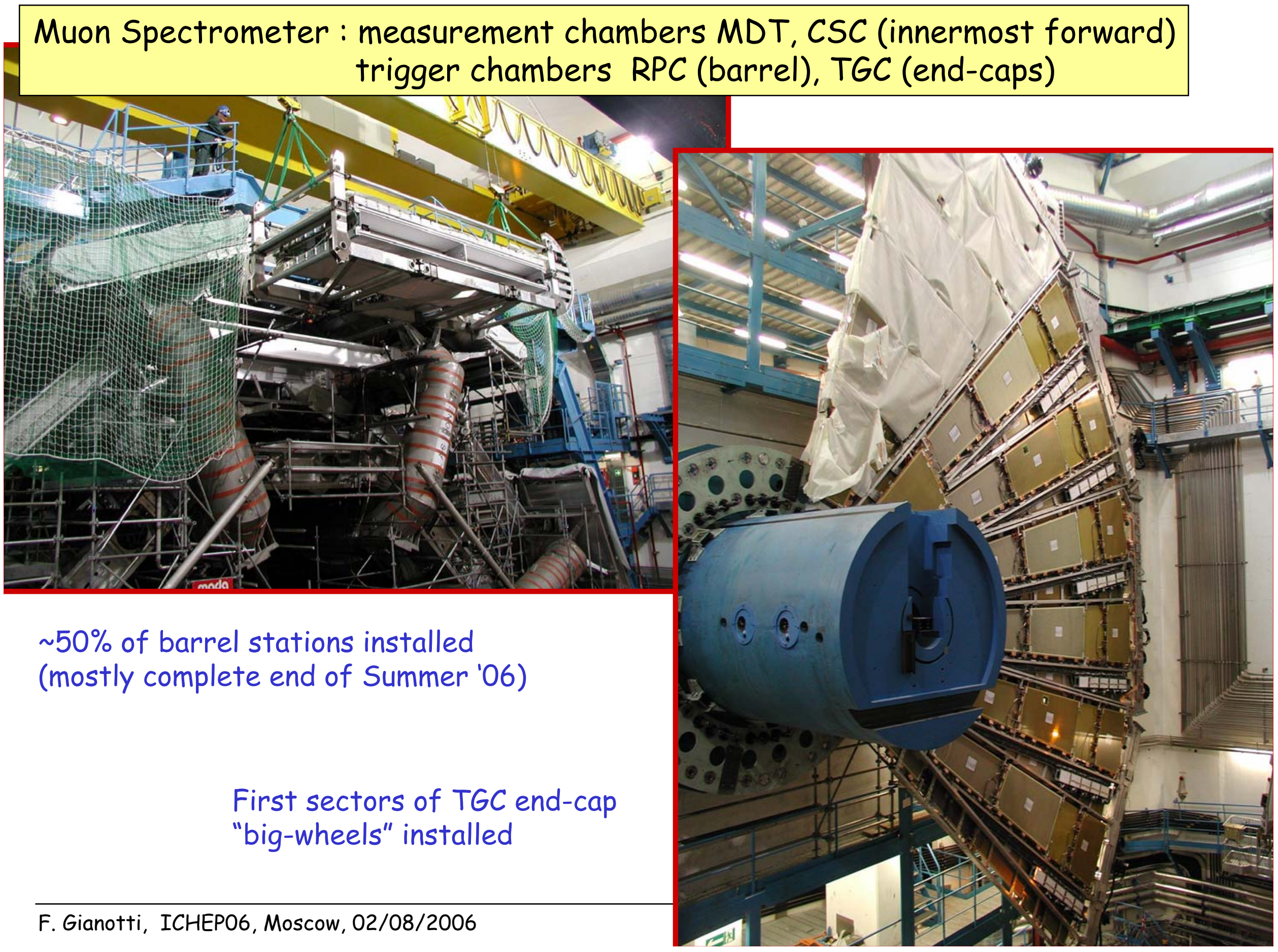


First cosmics have been registered in the underground cavern with barrel Muon chambers (MDT and RPC) and Level-1 $\mu$ trigger
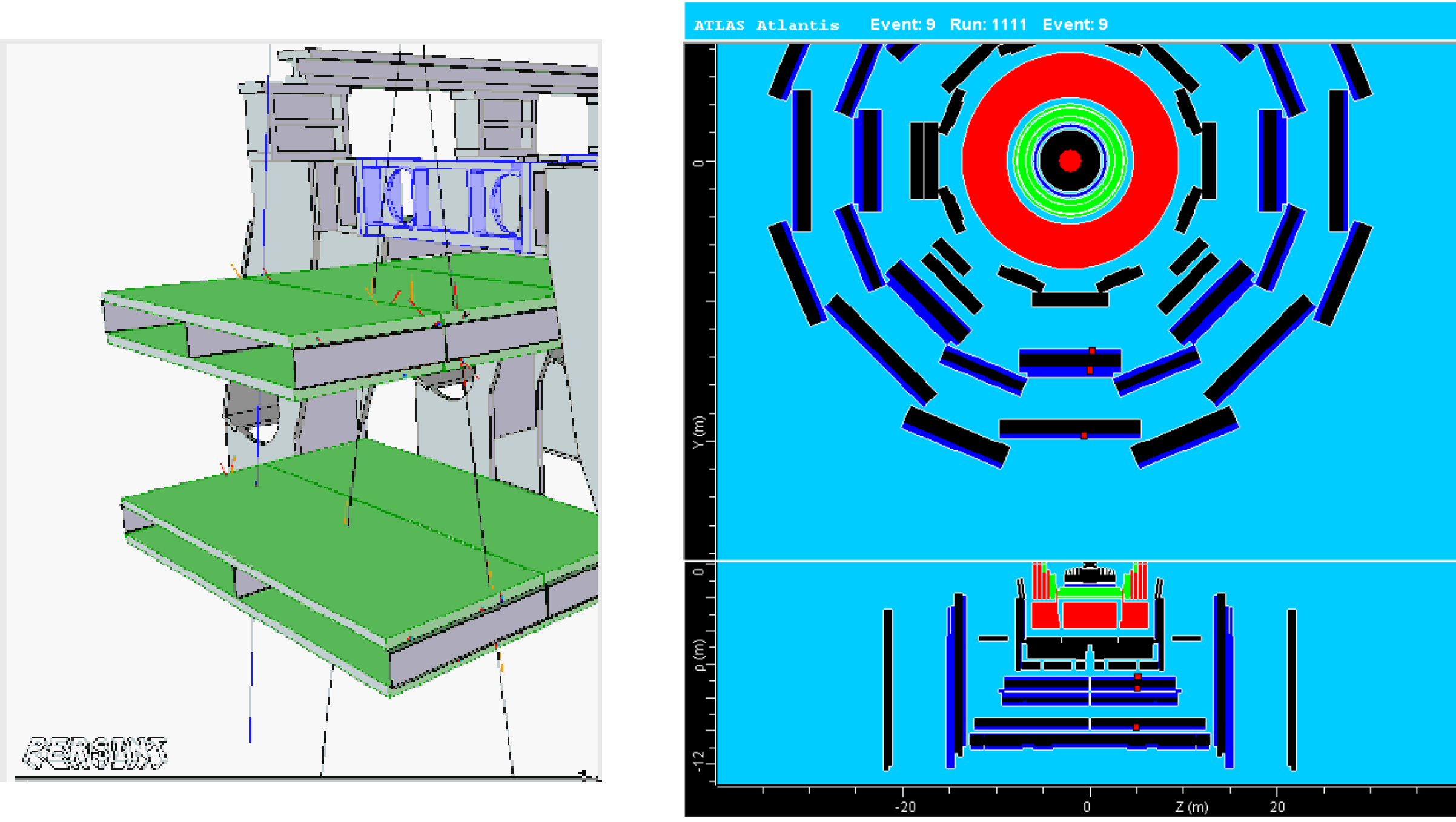

F. Gianotti, ICHEPO6, Moscow, 02/08/2006 


\section{Towards Physics (1): the 2004 ATLAS combined test beam}

Full "vertical slice" of ATLAS tested on CERN H8 beam line May-November 2004

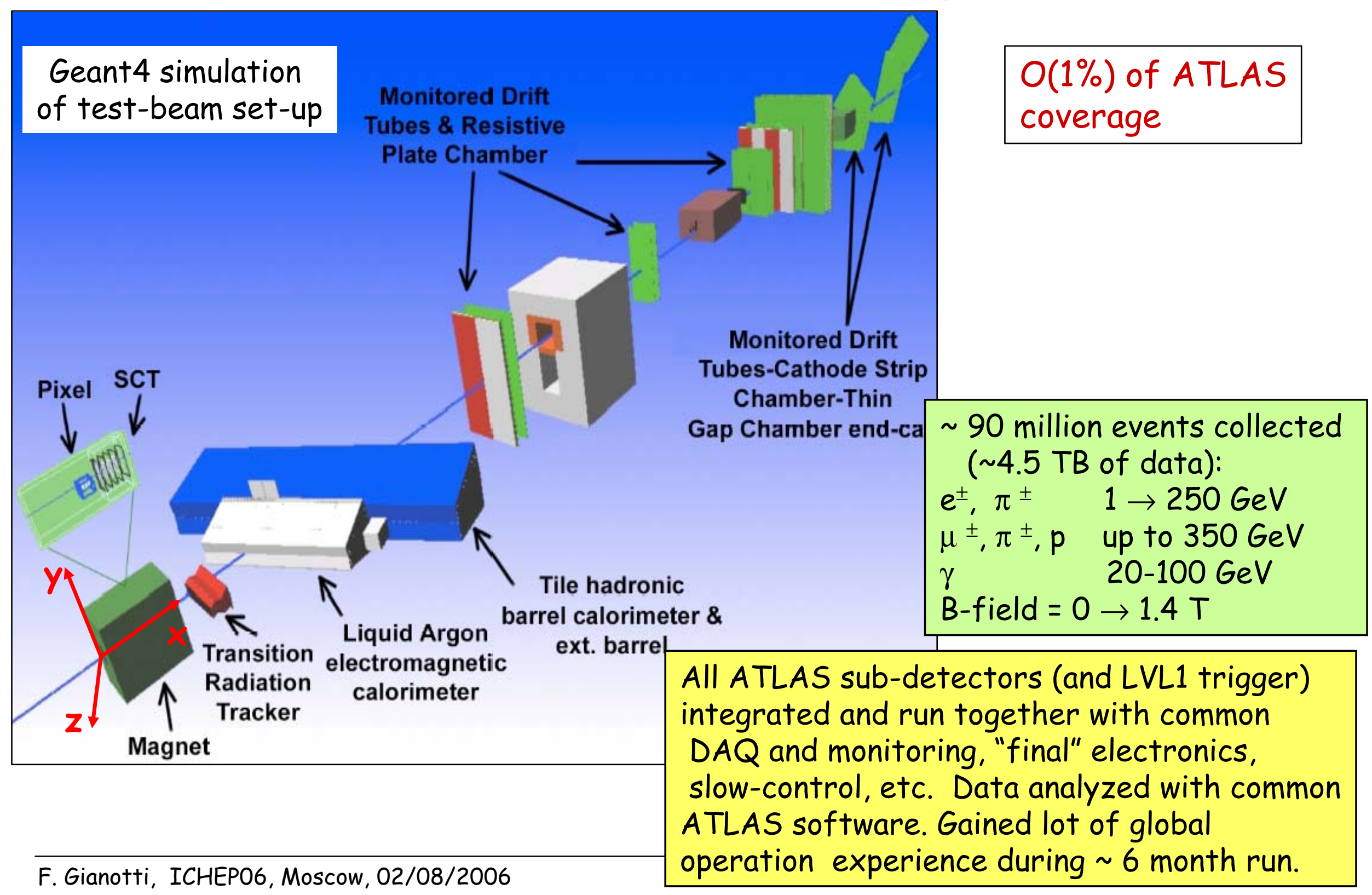



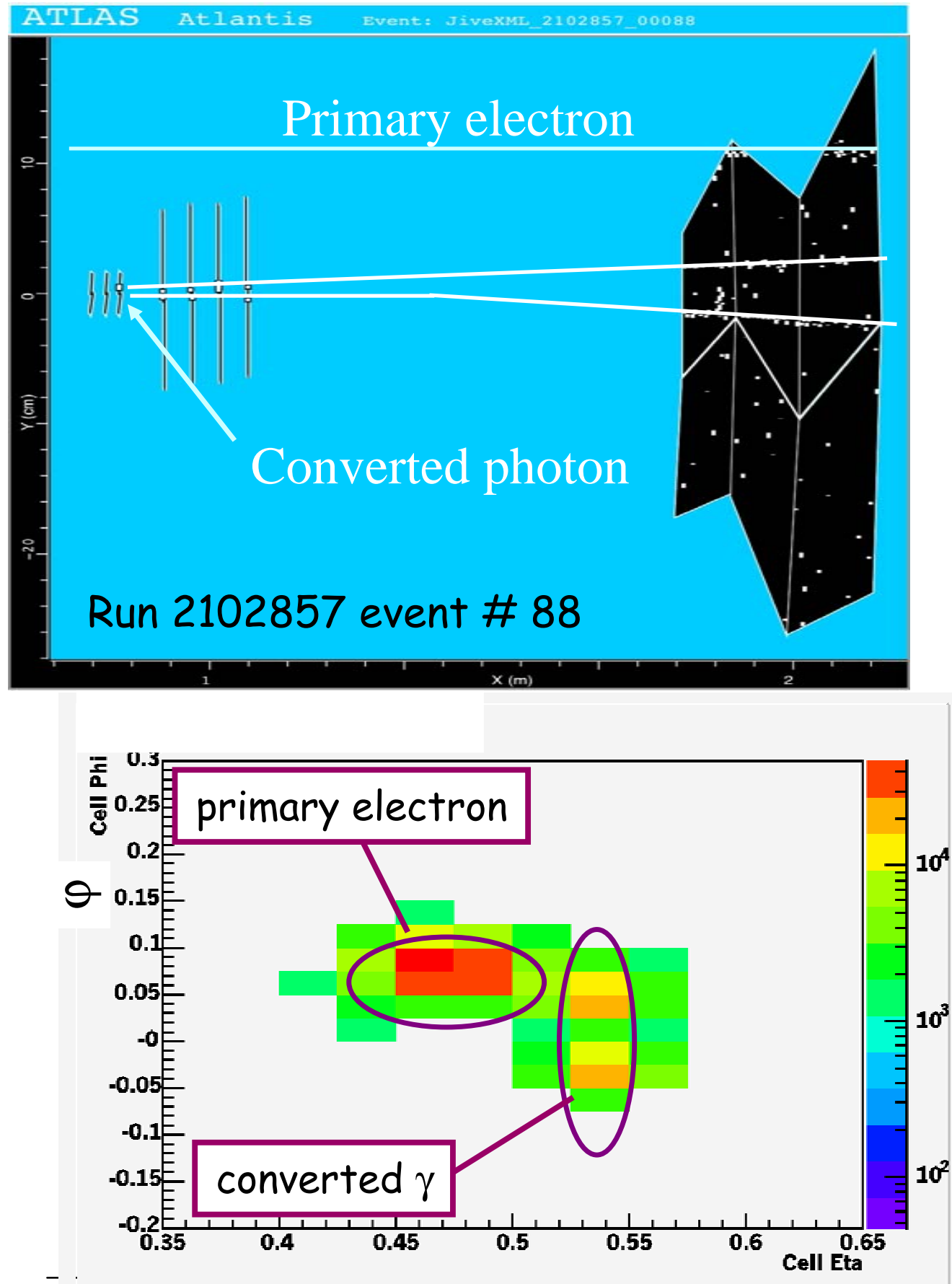

F. Gianotti, ICHEP06, Moscow, 02/08/2006

\section{ATLAS preliminary}

ATLAS @ LHC:

$\gamma$-conversion probability in tracker is $>30 \% \rightarrow$ important to develop

(and validate!) efficient

reconstruction tools

\section{Inner Detector tracks extrapolated}

to ECAL and compared to calo clusters

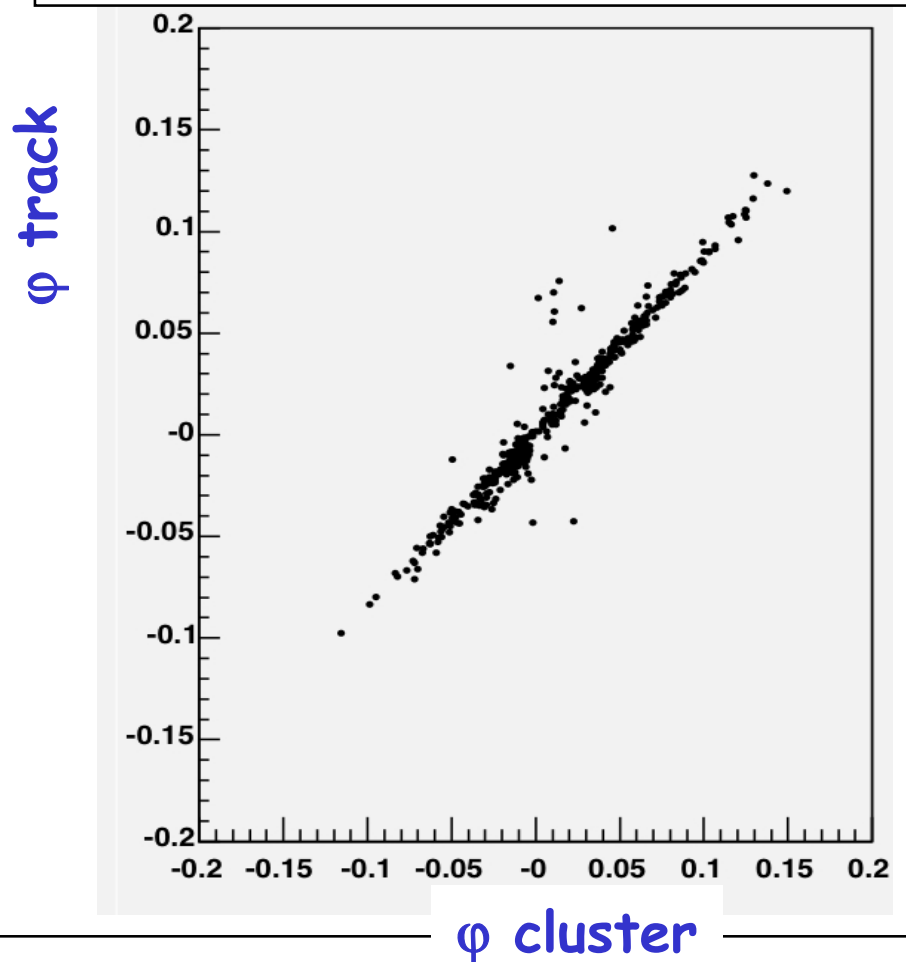




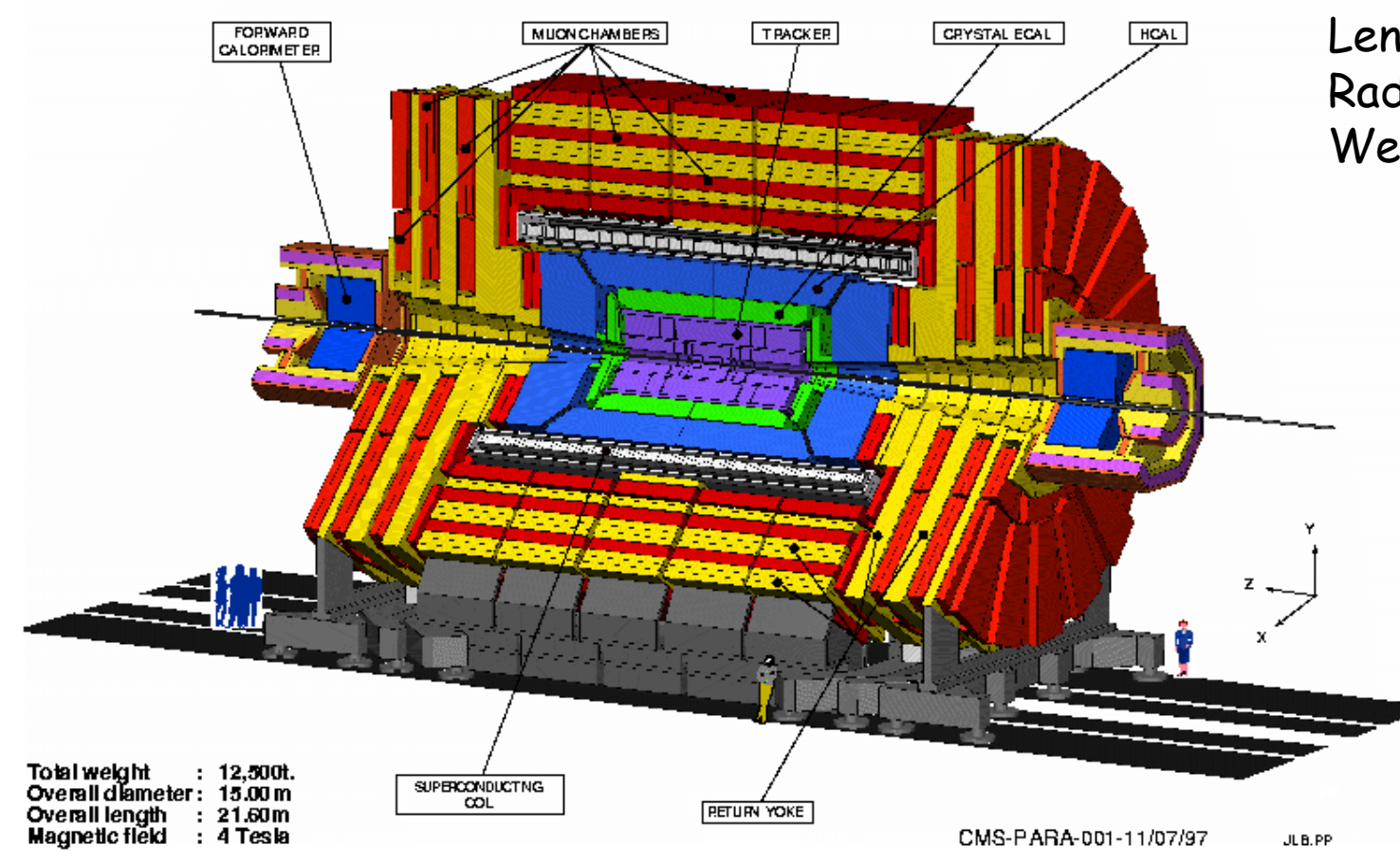

Length : 22 m

Radius : $\sim 7 \mathrm{~m}$

Weight : 12500 tons

Compact and modular: assembled at the surface and lowered in the cavern piece by piece

- Tracking $(|\eta|<2.5, B=4 T)$ : Si pixels and strips

- Calorimetry $(|\eta|<5)$ :

-- EM : $\mathrm{PbWO}_{4}$ crystals

-- HAD: brass/scintillator (central+ end-cap), Fe/Quartz (fwd)

- Muon Spectrometer $(|\eta|<2.5)$ : return yoke of solenoid instrumented with muon chambers

YBO lowering (2000t): Dec. 2006

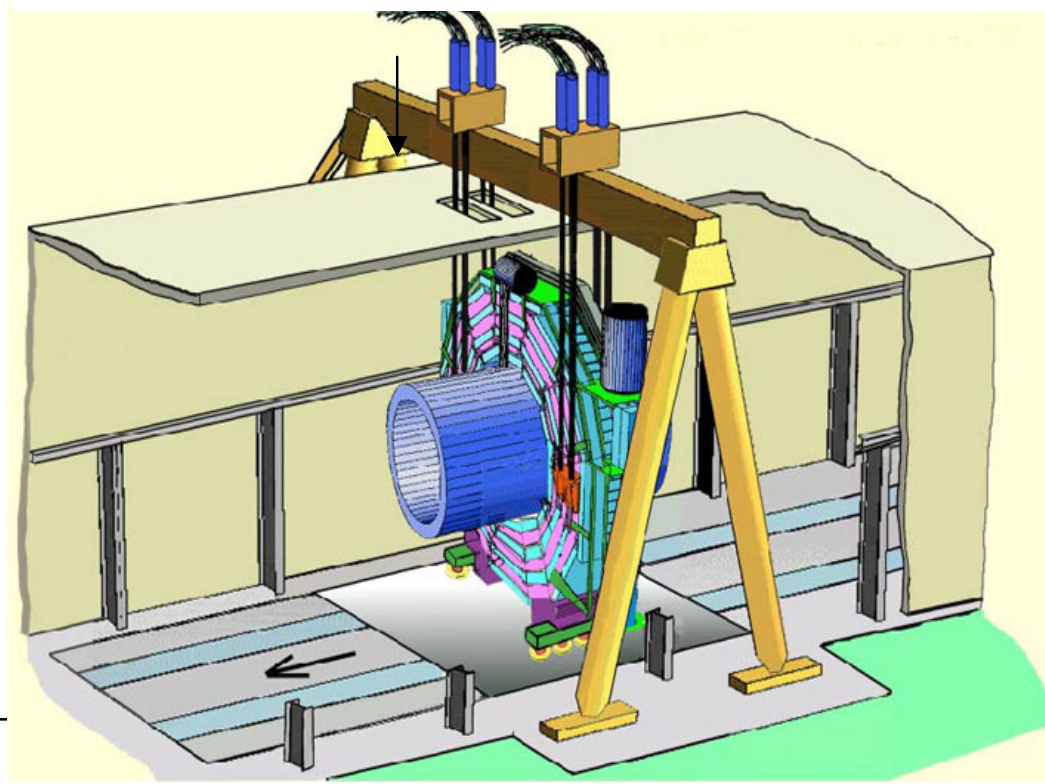


At the surface, solenoid inserted on 14 Sept. 2005; cooled down to $4.5 \mathrm{~K}$ in February 2006:

ramping up the current, now at $12.5 \mathrm{kA}(2.5 \mathrm{~T})$

$\rightarrow$ magnetic test/field map starting Aug./Sept. 2006 (MTCC)

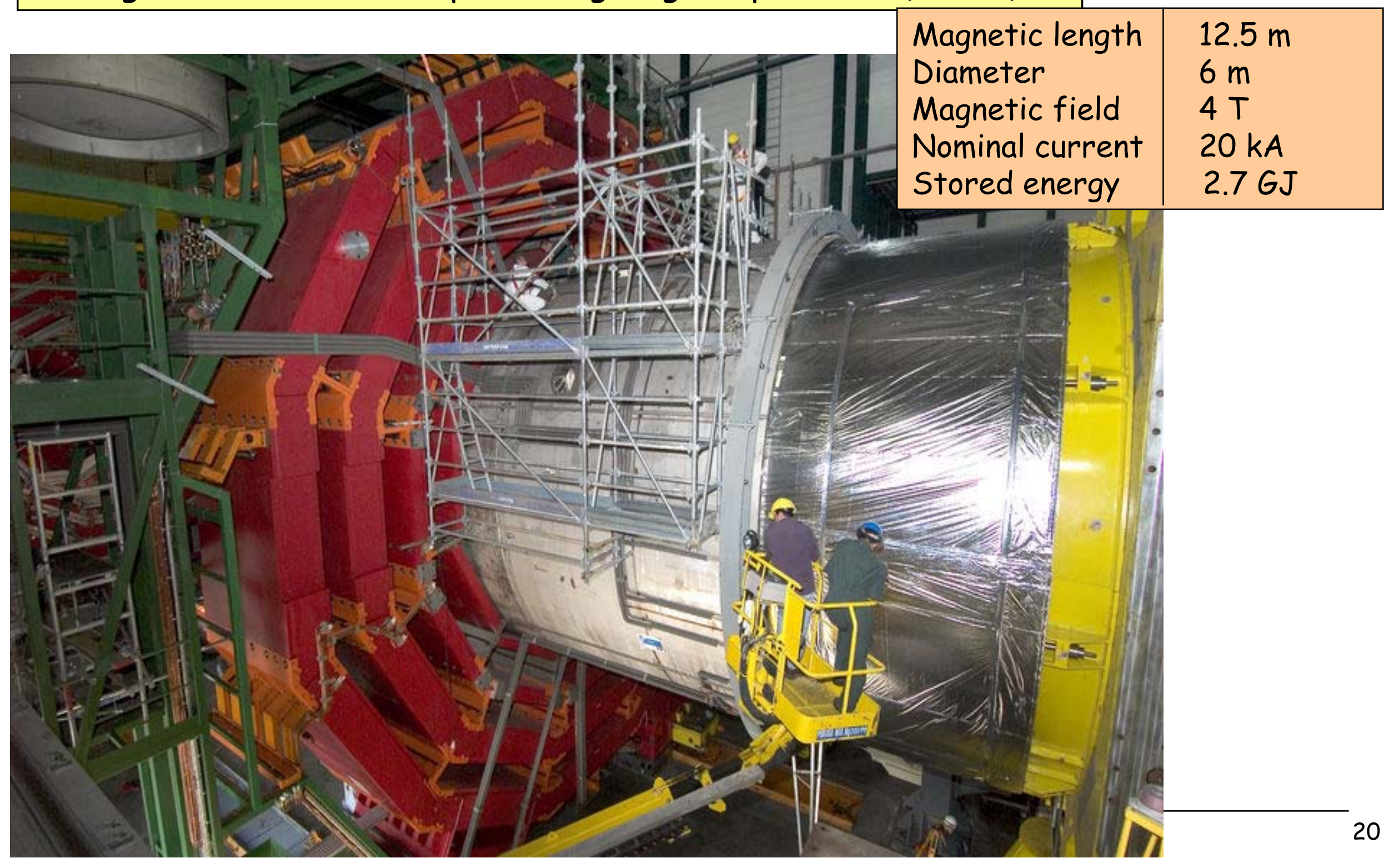


Installation of Muon chambers : 90\% complete in end-cap (CSC+RPC), 60\% in barrel (DT+RPC)

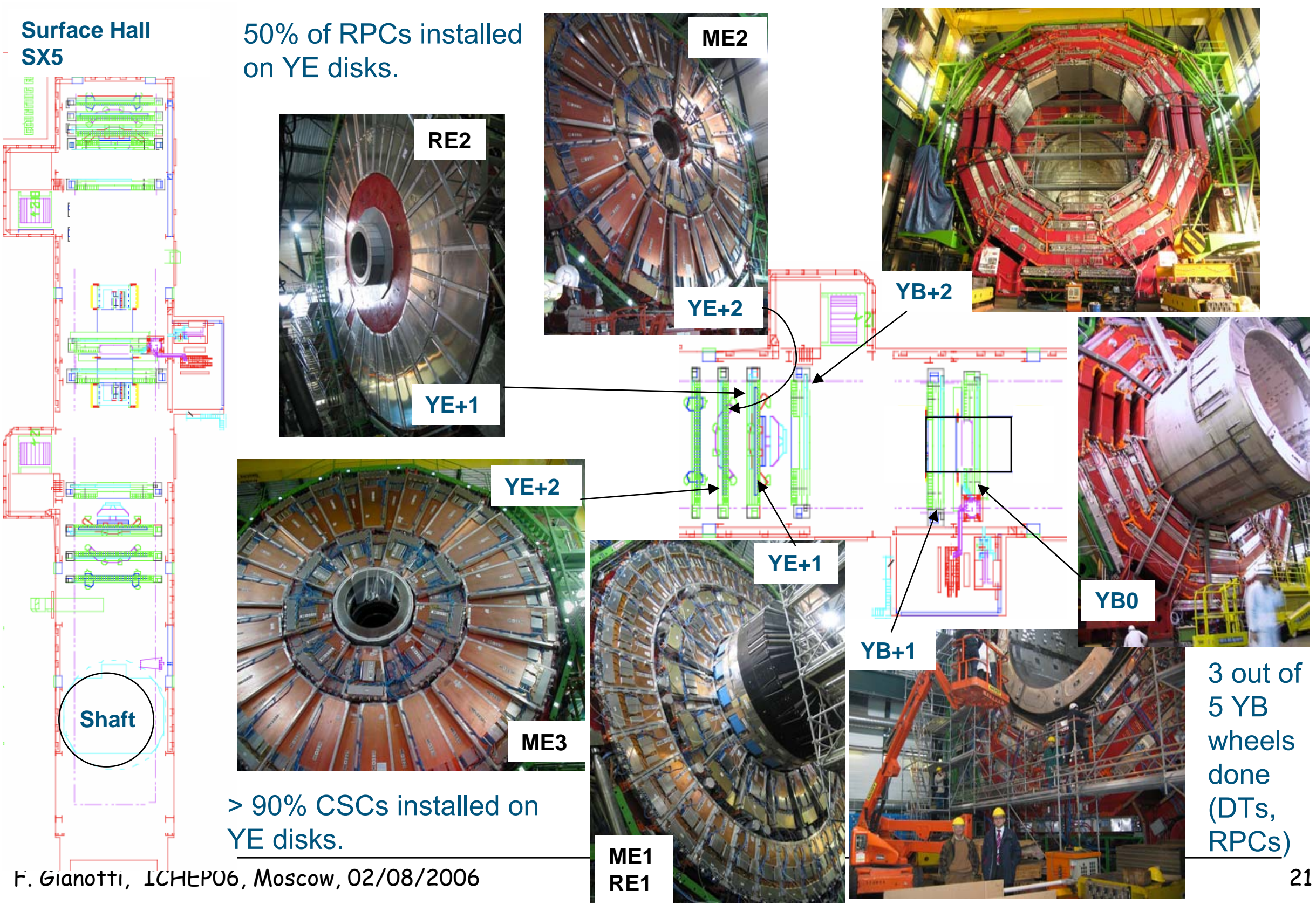




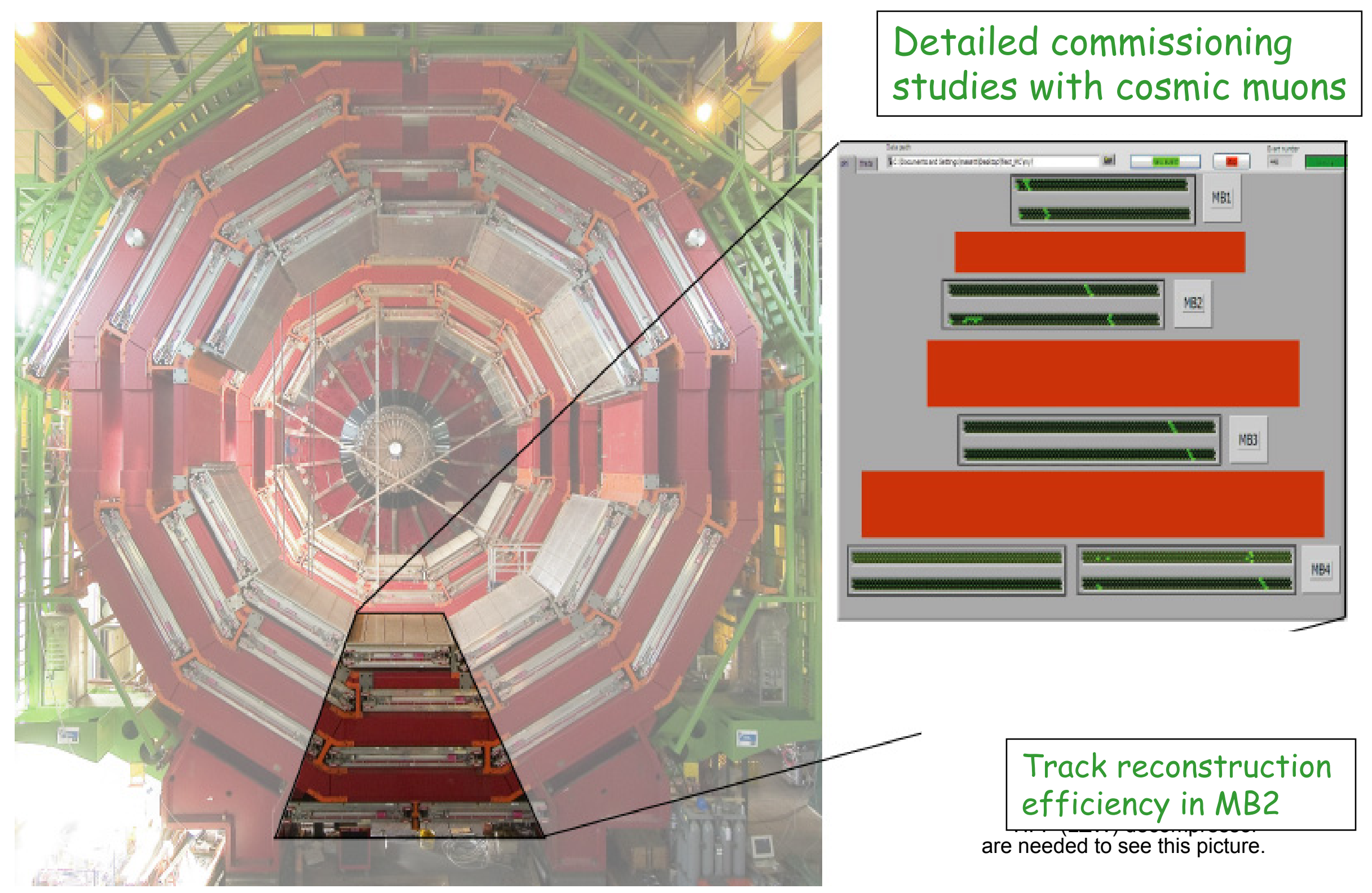

F. Gianotti, ICHEPO6, Moscow, 02/08/2006 
Cosmic muon bending in the CMS return yoke, collected yesterday with 12.5kA in the solenoid $(B=2.5 T)$

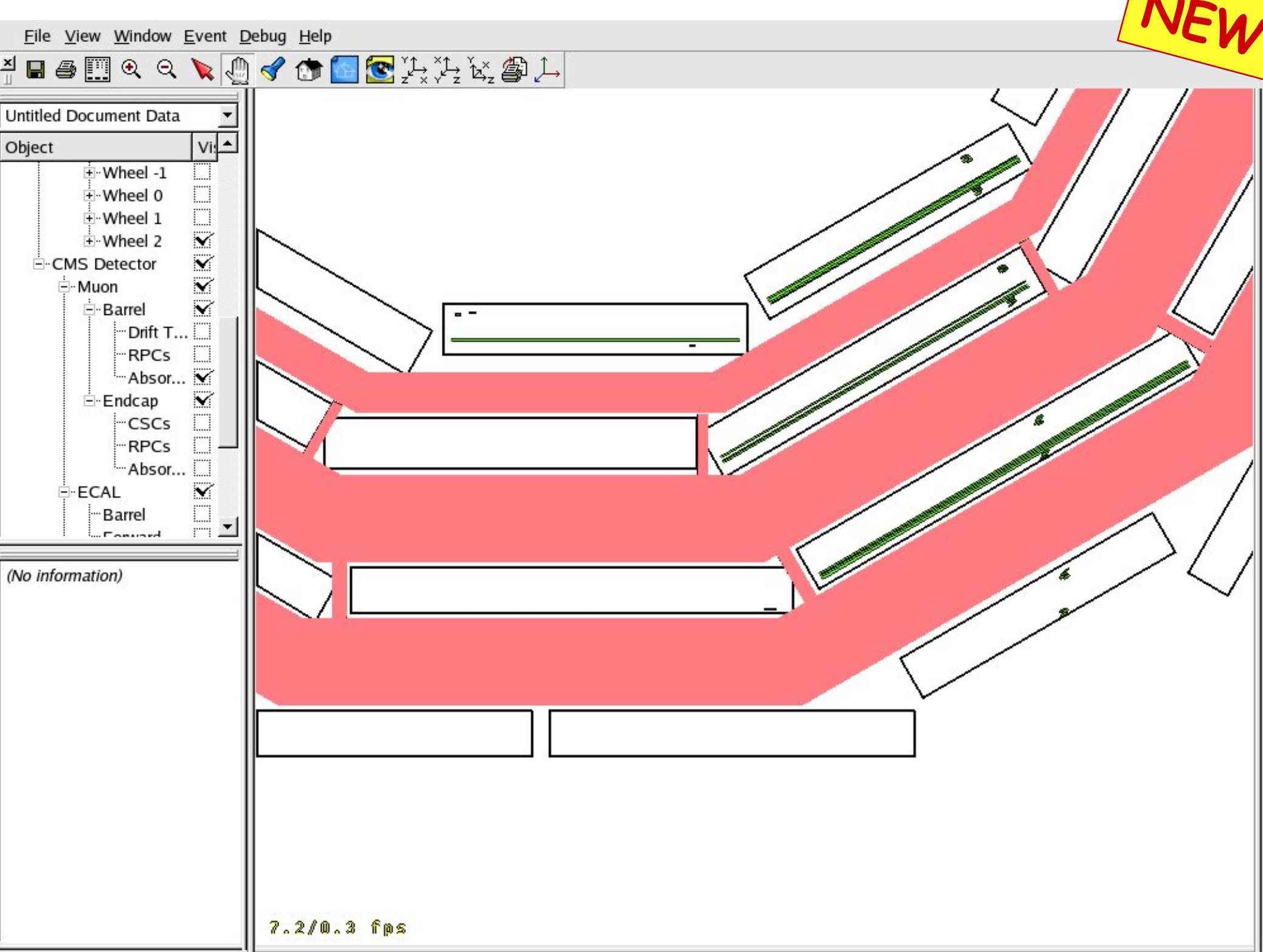

Run \# 0, event \# 210 


\section{Electromagnetic calorimeter}

Barrel : 36 SuperModules (SM), 1700 crystals each Total of 61000 barrel crystals ( $85 \%$ delivered)

27 bare SM assembled, 22 equipped with electronics

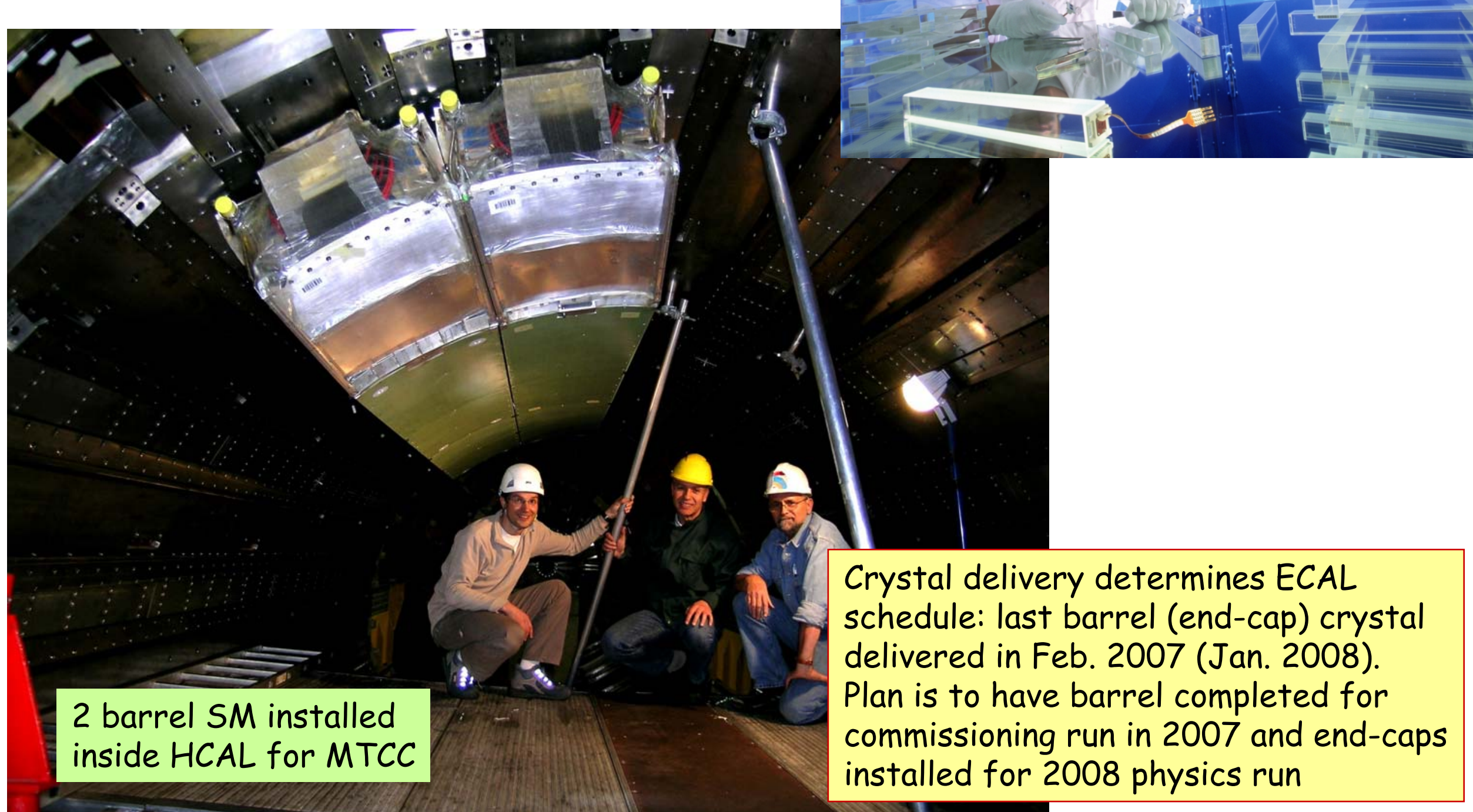


Inner tracker:

$\sim 220 \mathrm{~m}^{2}$ of Si sensors 10.6 million Si strips 65.9 million Pixels

- Assembly of all 16000 modules completed

- Integration progressing well

- Installation at Point 5 in April 2007

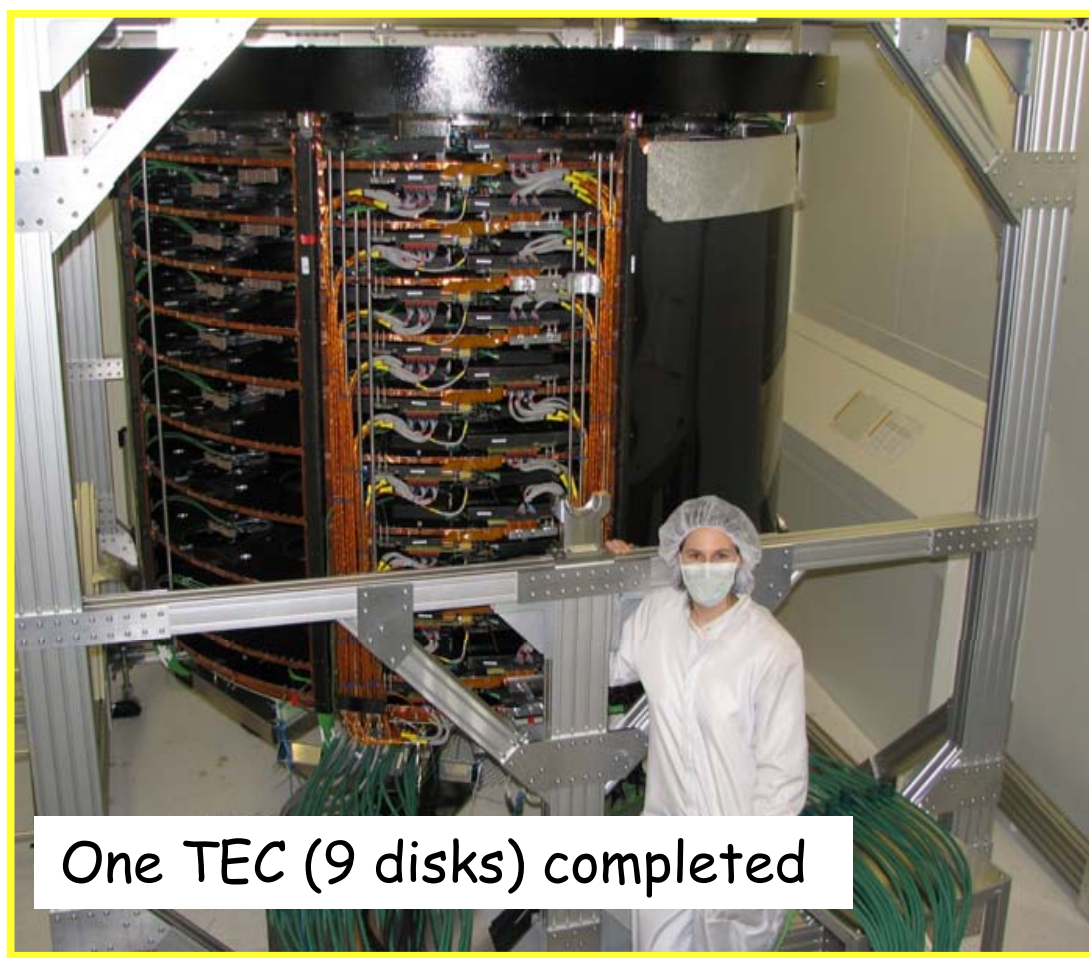

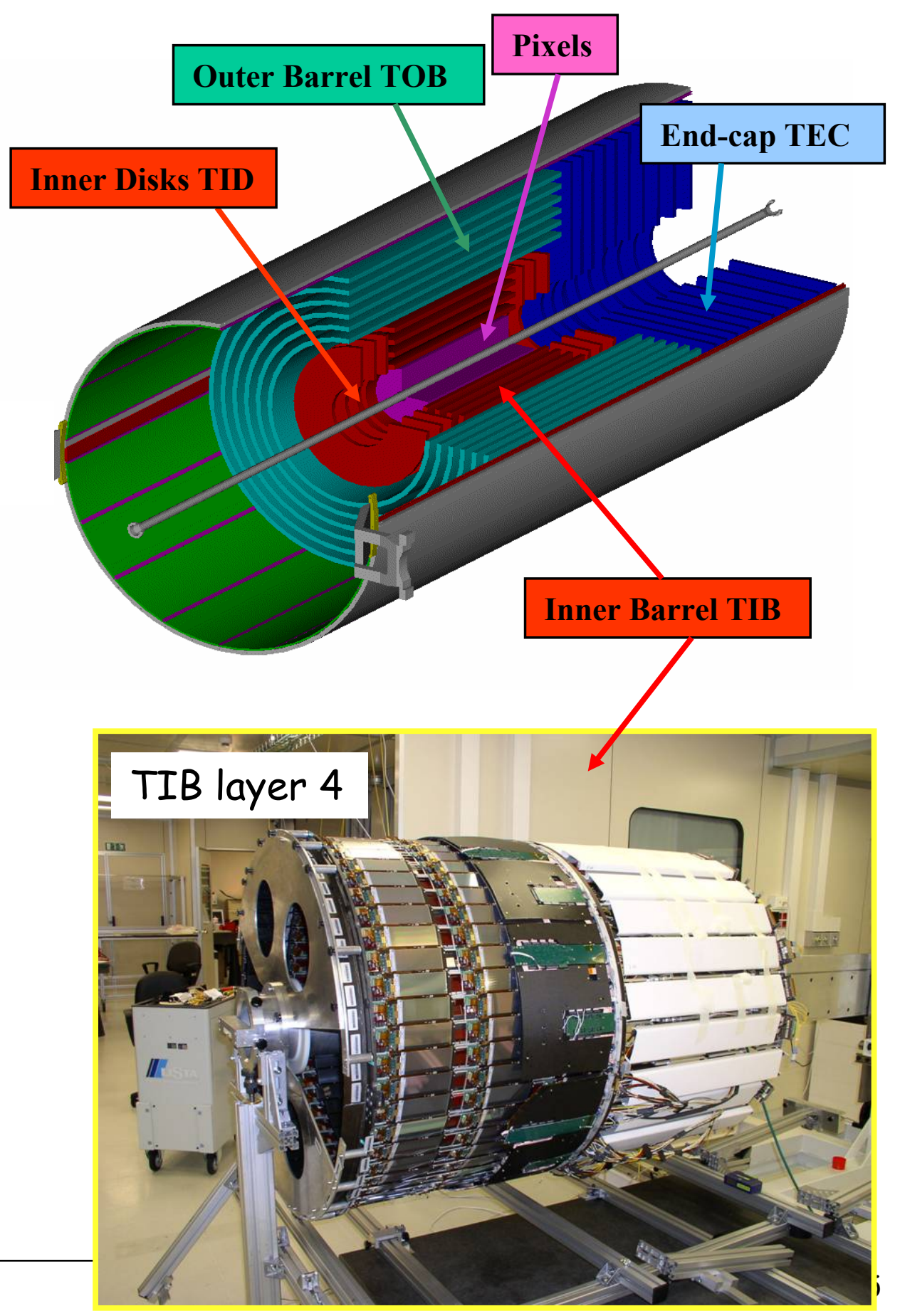


Towards Physics (2): the CMS Magnet Test and Cosmic Challenge (MTCC)

Cosmics run of a full detector slice (few percent of CMS coverage) inside $4 T$ field. Magnet being energized, detector closed, data taking started ...

Test: detector installation and closing; magnet commissioning and field map; combined operation of full chain detector-electronics-DAQ-trigger-DCS-software identical to final experiment; timing, calibration, alignment procedures

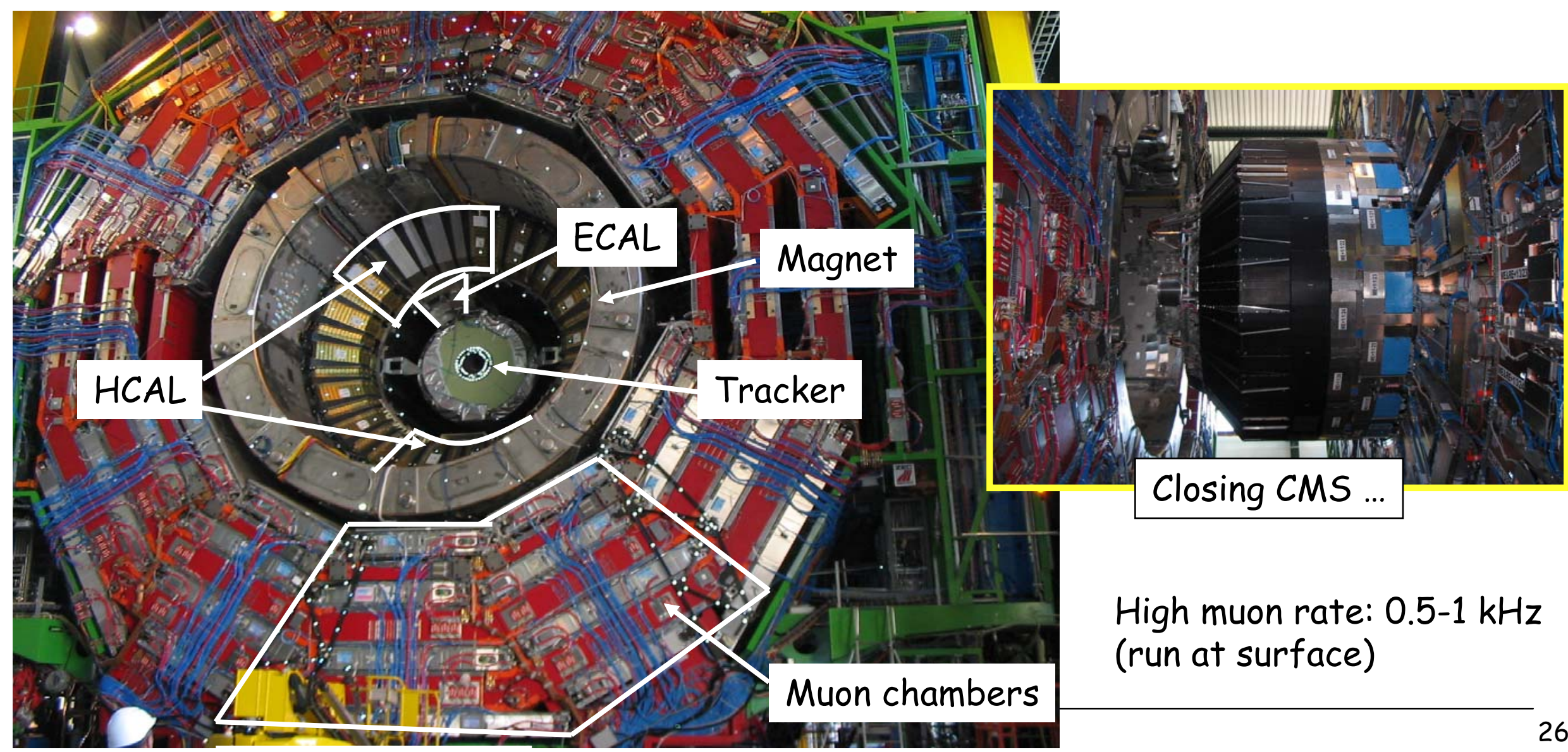




\section{CMS Physics Technical Design Reports}
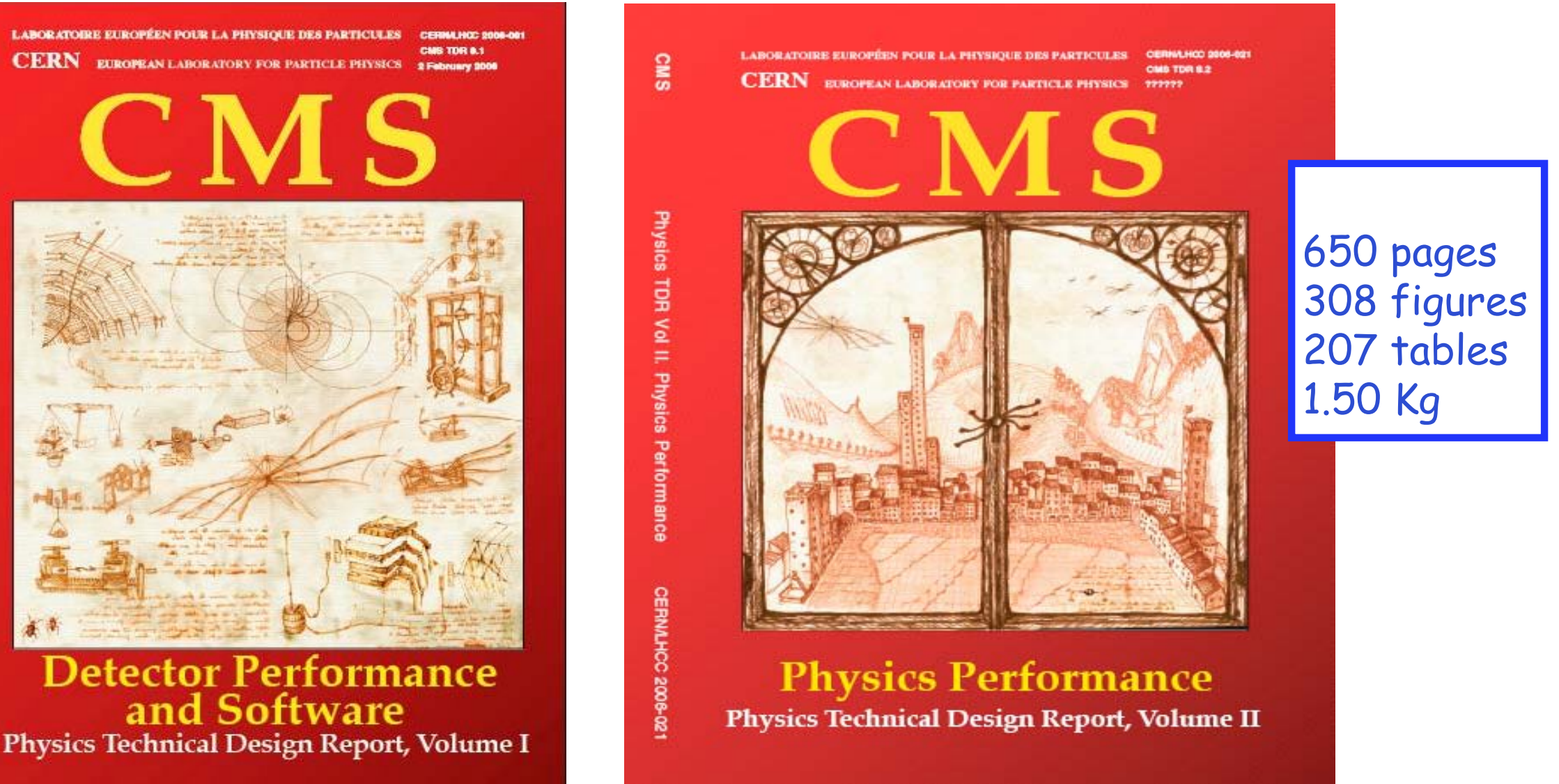

hittp://cmsdoc.cerm.ch/cms/cpt/tdr/

CERN/LHCC 2006-001

February 2006
CERN/LHCC 2006-021

June 2006 


\section{(C) First physics with first data}

- Understand detector and Standard Model physics

- Discoveries?

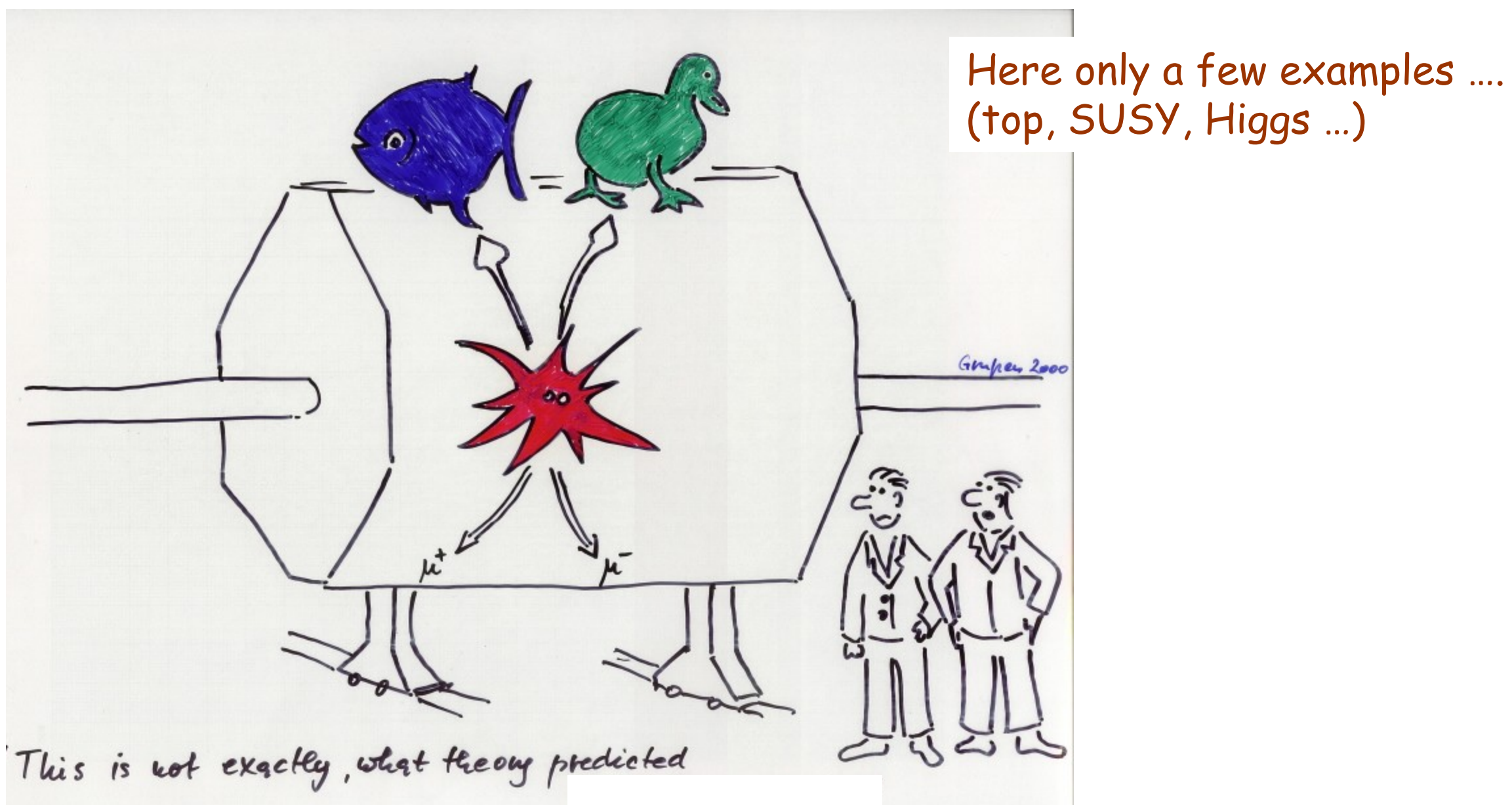


ATLAS preliminary $\sqrt{ } s=900 \mathrm{GeV}, \mathrm{L}=10^{29} \mathrm{~cm}^{-2} \mathrm{~s}^{-1}$ efficiencies included

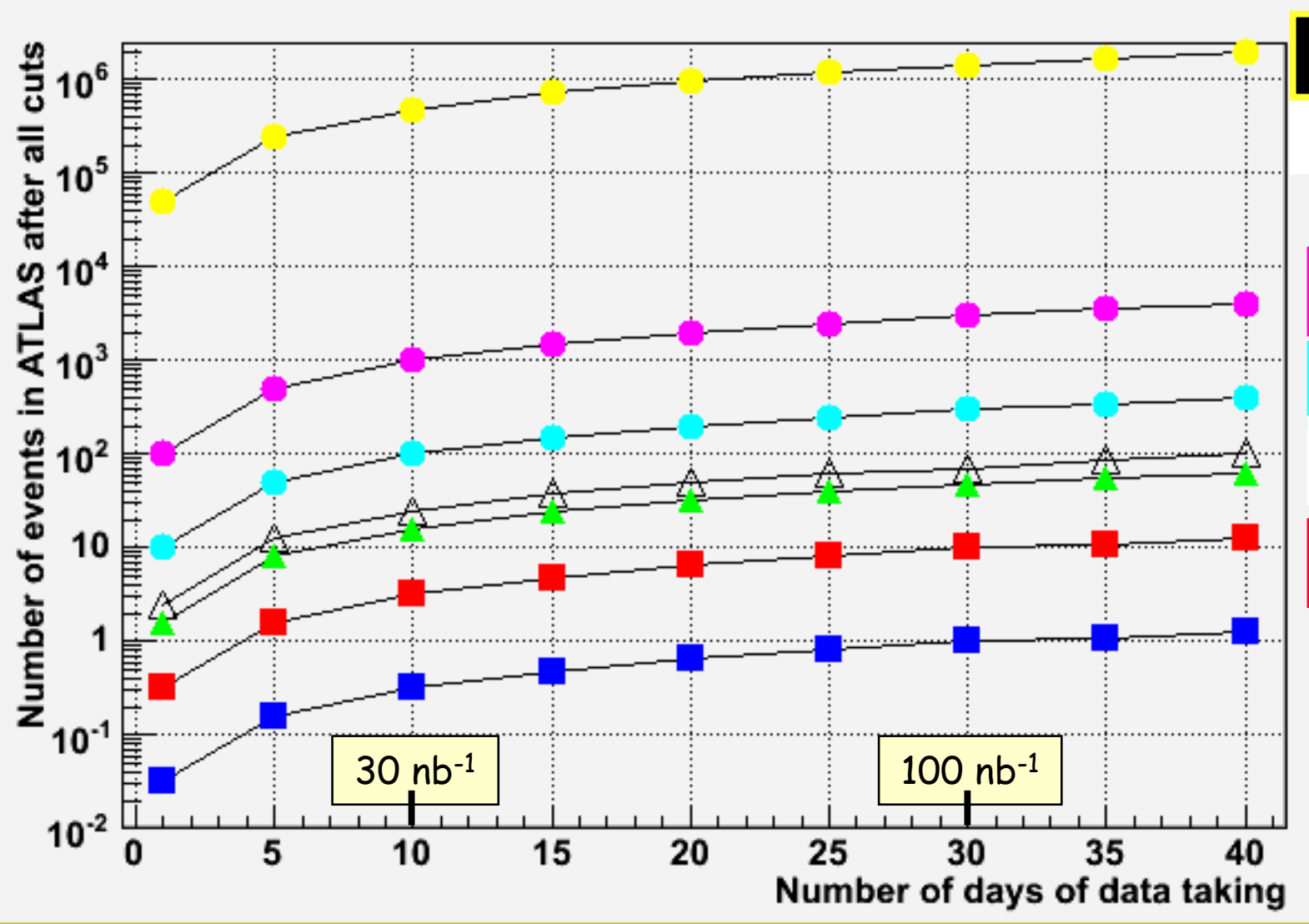

\section{Jets $p_{T}>15 \mathrm{GeV}$}

(b-jets: $1.5 \%)$

\section{Jets $p_{T}>50 \mathrm{GeV}$}

Jets $\mathrm{p}_{\mathrm{T}}>70 \mathrm{GeV}$

$\mathrm{Y} \rightarrow \mu \mu \mathrm{J} / \psi \rightarrow \mu \mu$

\section{$W \rightarrow e v, \mu \nu$}

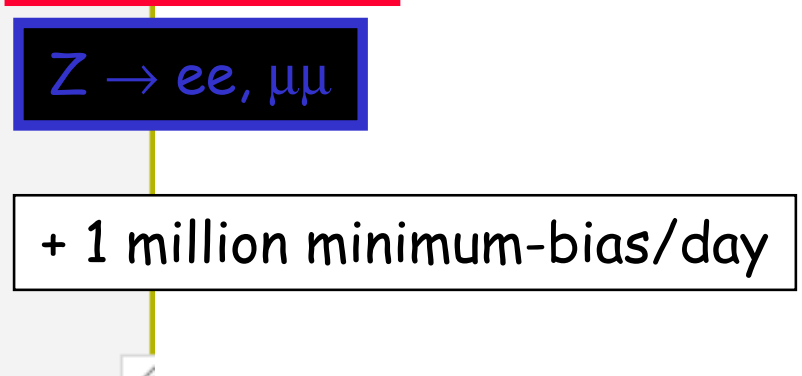

Start to commission triggers and detectors with collision data (minimum bias, jets, ..) in real LHC environment

- Maybe first physics measurements (minimum-bias, underlying event, QCD jets, ...)?

- Observe a few $\mathrm{W} \rightarrow \mathrm{I}, \mathrm{Y} \rightarrow \mu \mu, \mathrm{J} / \psi \rightarrow \mu \mu$ ? 


\section{Example 1: CMS ECAL calibration}

only 6 SM will be tested with test beams $\rightarrow$ most/all SM will be calibrated with cosmic rays
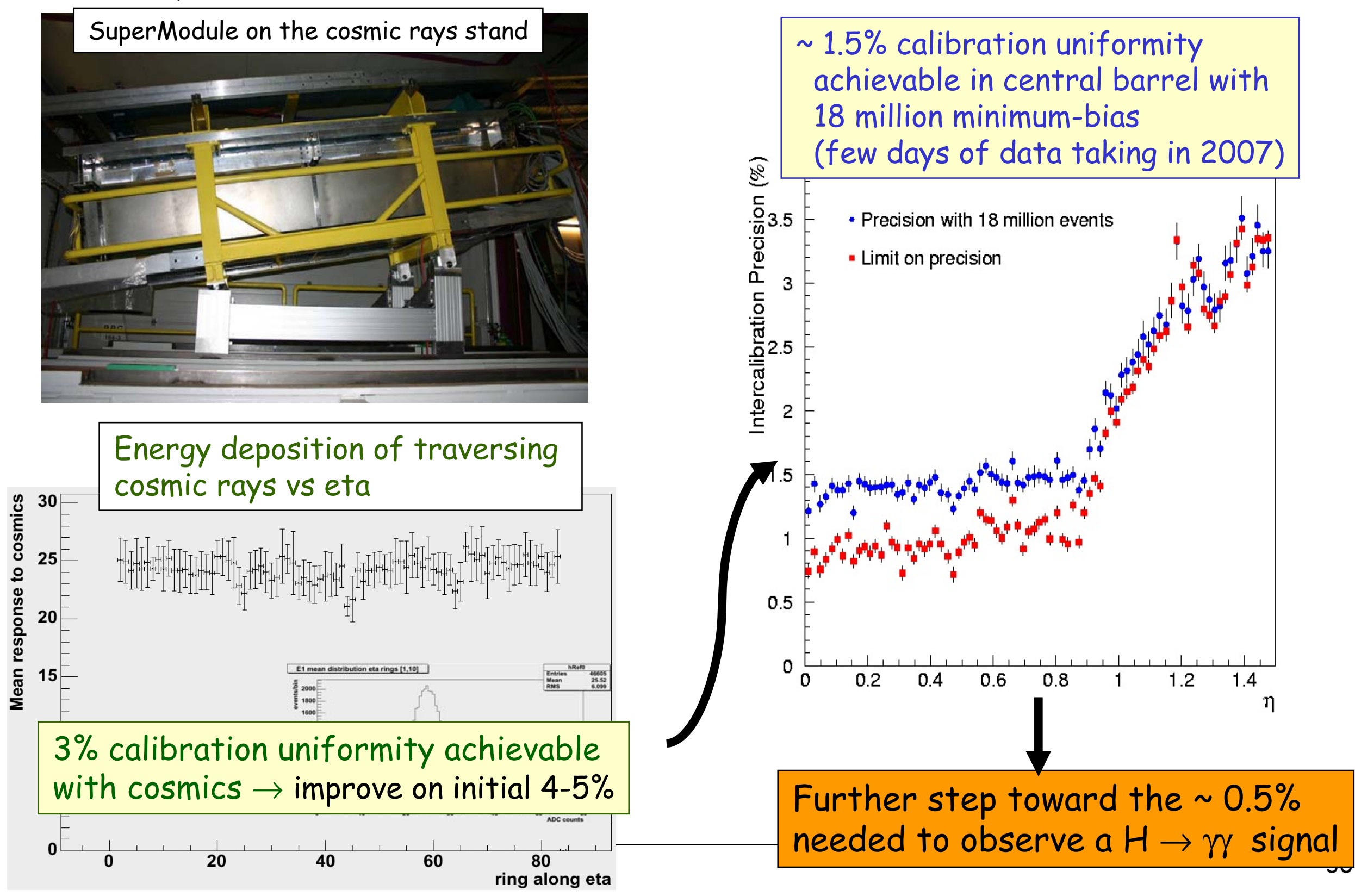


\section{Example 2: Measurement of the underlying event in di-jet production}

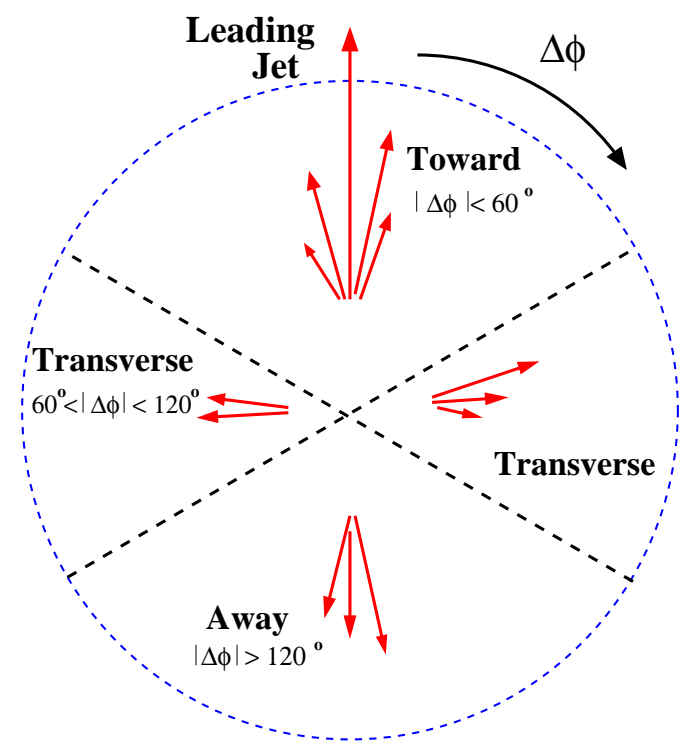

Multiplicity of charged particles with $\mathrm{p}_{T}>0.5 \mathrm{GeV}$ and $|\eta|<1$ in region transverse to leading jet

Particle multiplicity of underlying event obtained from the region transverse to the leading jet.

Comparison of plateau's between LHC and Tevatron will tell if detector performance, reconstruction tools and physics are under control

F. Gianotti, ICHEP06, Moscow, 02/08/2006

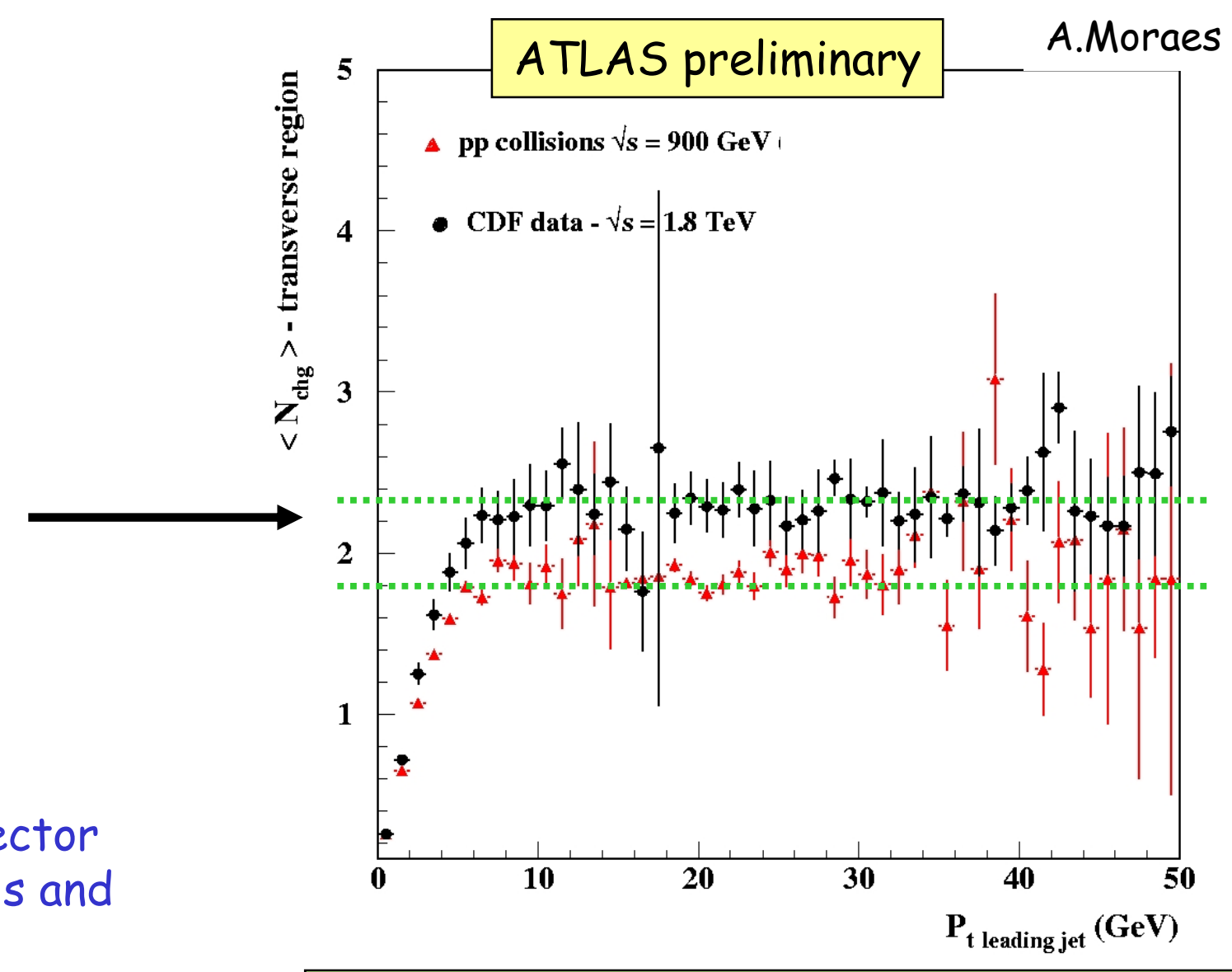

$\sim 15$ days of data taking in 2007 enough to cover up to $\mathrm{p}_{\mathrm{T}}$ (leading jet) $\sim 40 \mathrm{GeV}$ 


\section{With the first physics run in $2008(\sqrt{s}=14 \mathrm{TeV}) \ldots$}

$1 \mathrm{fb}^{-1}\left(100 \mathrm{pb}^{-1}\right) \equiv 6$ months (few days) at $L=10^{32} \mathrm{~cm}^{-2} \mathrm{~s}^{-1}$ with $50 \%$ data-taking efficiency

$\rightarrow$ may collect a few $\mathrm{fb}^{-1}$ per experiment by end 2008

\begin{tabular}{|l|c|c|}
\hline Channels (examples ...) & $\begin{array}{c}\text { Events to tape for } 100 \mathrm{pb}^{-1} \\
\text { (per expt: ATLAS, CMS) }\end{array}$ & $\begin{array}{l}\text { Total statistics from } \\
\text { some of previous Colliders }\end{array}$ \\
\hline $\begin{array}{l}W \rightarrow \mu v \\
\sim 10^{4} \mathrm{LEP}, \sim 10^{6} \text { Tevatron }\end{array}$ & $\sim 10^{6}$ \\
$Z \rightarrow \mu \mu$ & $\sim 10^{6} \mathrm{LEP}, \sim 10^{5}$ Tevatron \\
$\tilde{g} \tilde{g}$ & $\sim 10^{5}$ & $\sim 10^{4}$ Tevatron \\
t† $\rightarrow \mathrm{Wb} \mathrm{Wb} \rightarrow \mu v+X$ & $\sim 10^{4}$ & --- \\
QCD jets $\mathrm{p}_{T}>1 \mathrm{TeV}$ & $>10^{3}$ & --
\end{tabular}

- Understand and calibrate detectors in situ using well-known physics samples e.g. $-Z \rightarrow e e, \mu \mu$ tracker, ECAL, Muon chambers calibration and alignment, etc.

- t† $\rightarrow$ blv bjj jet scale from $W \rightarrow j j$, b-tag performance, etc.

- Measure SM physics at $\sqrt{ } s=14 \mathrm{TeV}: \mathrm{W}, Z, \mathrm{tt}, \mathrm{QCD}$ jets ... (also because omnipresent backgrounds to New Physics)

F. Gianotti, ICH 
Example of initial measurement: understanding detector and physics with top events

Can we observe an early top signal with limited detector performance? And use it to understand detector and physics?

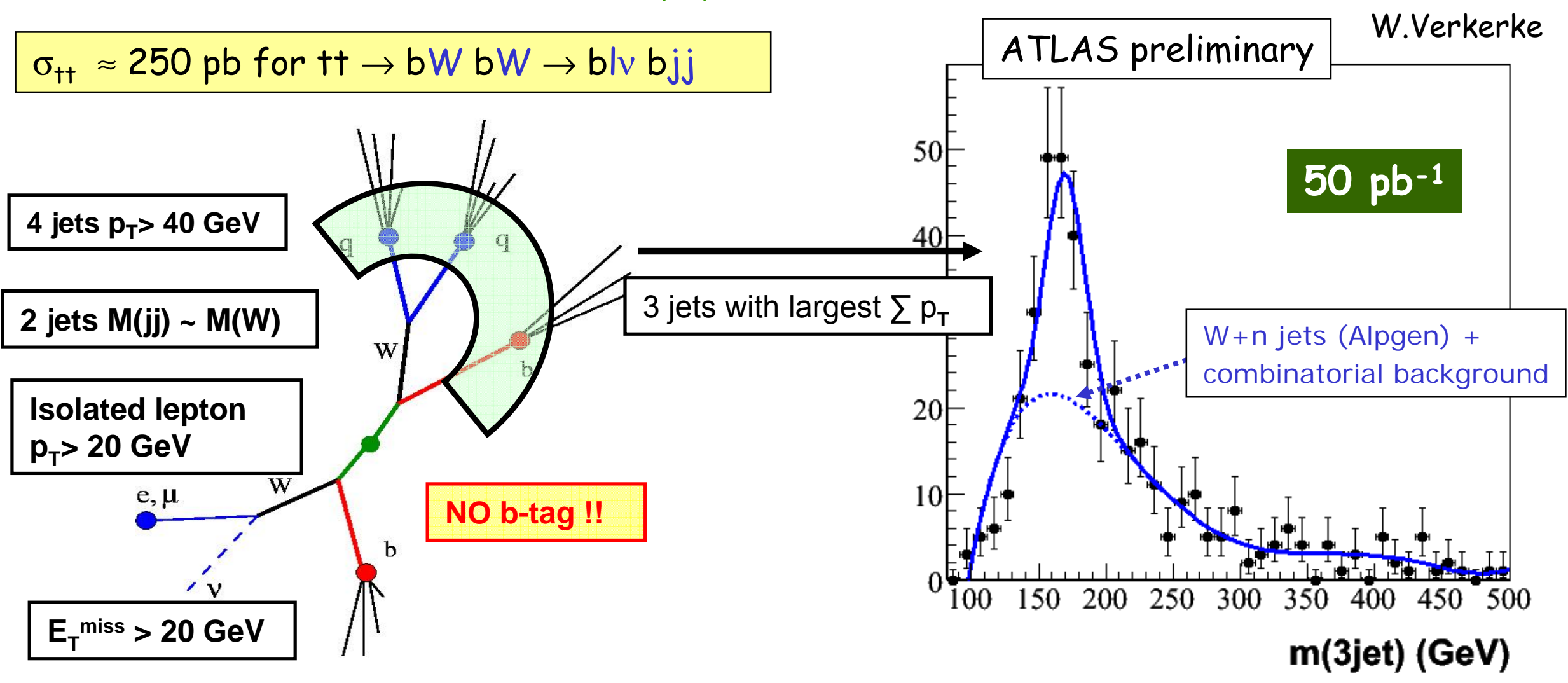

Top signal observable in early days with no b-tagging and simple analysis $\left(100 \pm 20\right.$ evts for $\left.50 \mathrm{pb}^{-1}\right) \rightarrow$ measure $\sigma_{t t}$ to $20 \%$, m to $10 \mathrm{GeV}$ with $\sim 100 \mathrm{pb}^{-1}$ ? In addition, excellent sample to:

- commission b-tagging, set jet $E$-scale using $W \rightarrow$ jj peak

- understand detector performance for $e, \mu$, jets, $b$-jets, missing $E_{T}, \ldots$

- understand / constrain theory and MC generators using e.g. $P_{T}$ spectra 


\section{Example of "early" discovery: Supersymmetry?}

If SUSY at TeV scale $\rightarrow$ could be found "quickly" .... thanks to:

- large $\quad \tilde{\mathrm{q}}, \tilde{\mathrm{g}}$ cross-section $\rightarrow \approx 10$ events/day at $10^{32}$ for $\mathrm{m}(\tilde{\mathrm{q}}, \tilde{\mathrm{g}}) \sim 1 \mathrm{TeV}$

- spectacular signatures (many jets, leptons, missing $E_{T}$ )
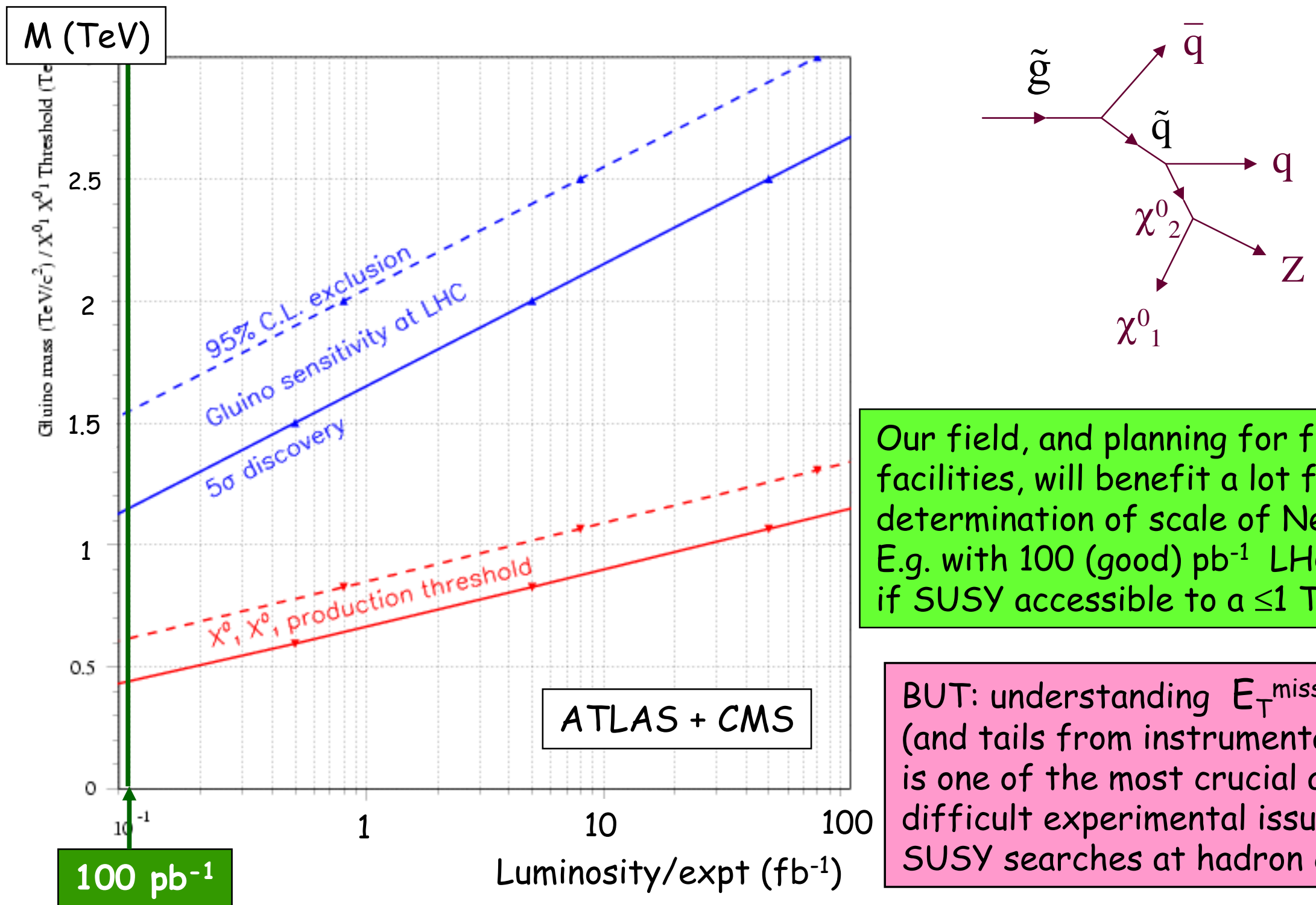

Our field, and planning for future facilities, will benefit a lot from quick determination of scale of New Physics. E.g. with 100 (good) $\mathrm{pb}^{-1}$ LHC could say if SUSY accessible to $a \leq 1 \mathrm{TeV}$ ILC

BUT: understanding $E_{T}$ miss spectrum (and tails from instrumental effects) is one of the most crucial and difficult experimental issue for SUSY searches at hadron colliders. 


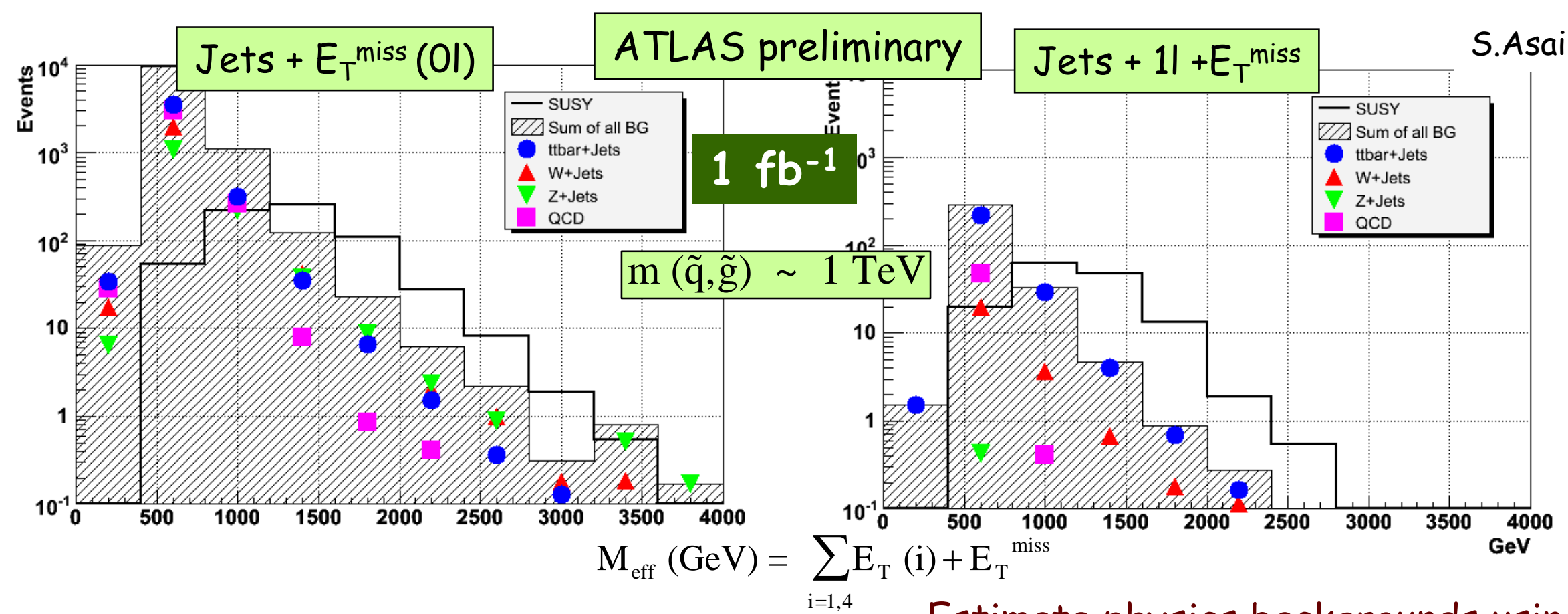

$E_{T}$ miss spectrum contaminated by cosmics, beam-halo, machine/detector problems, etc.

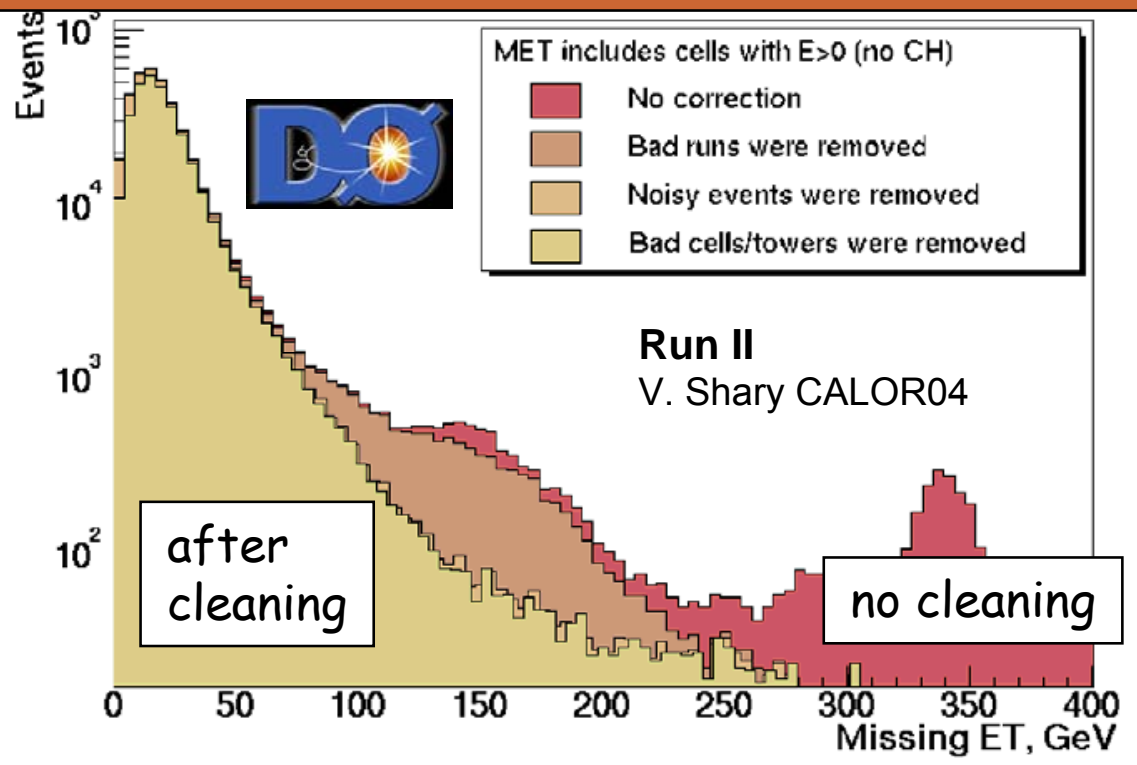

Estimate physics backgrounds using data (control samples)

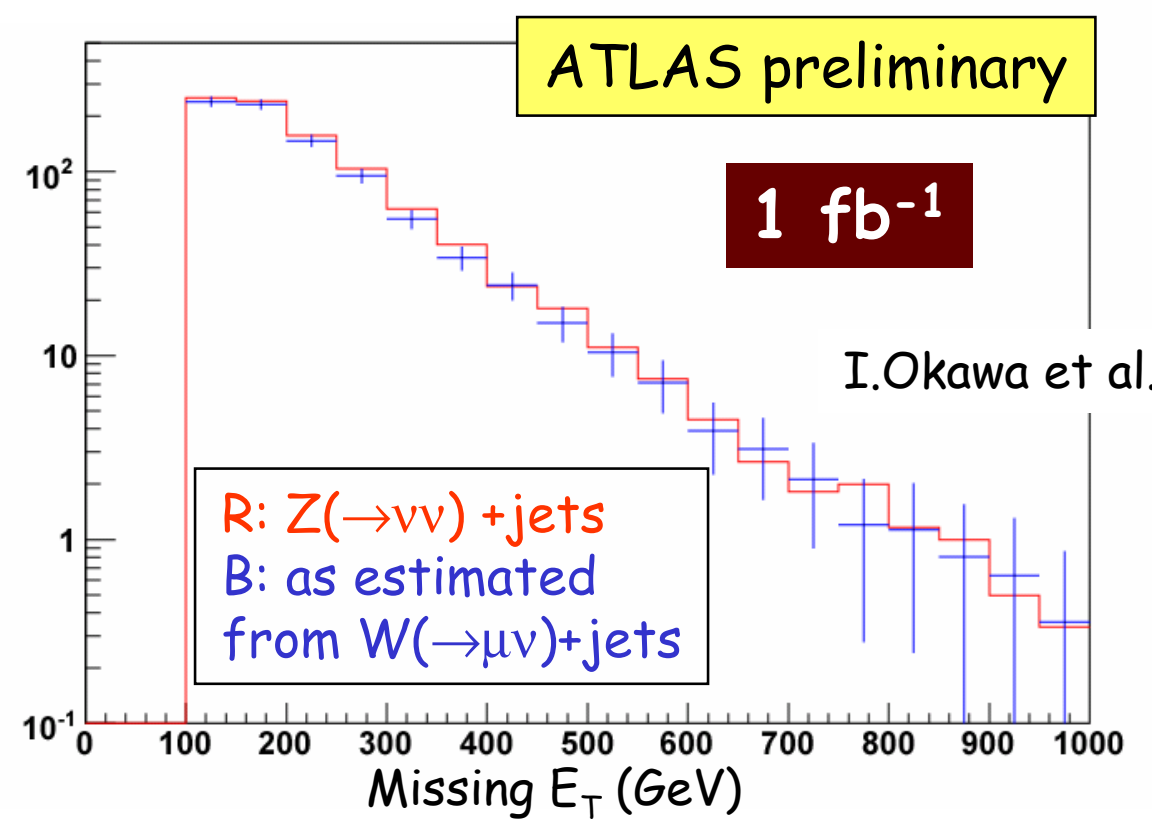




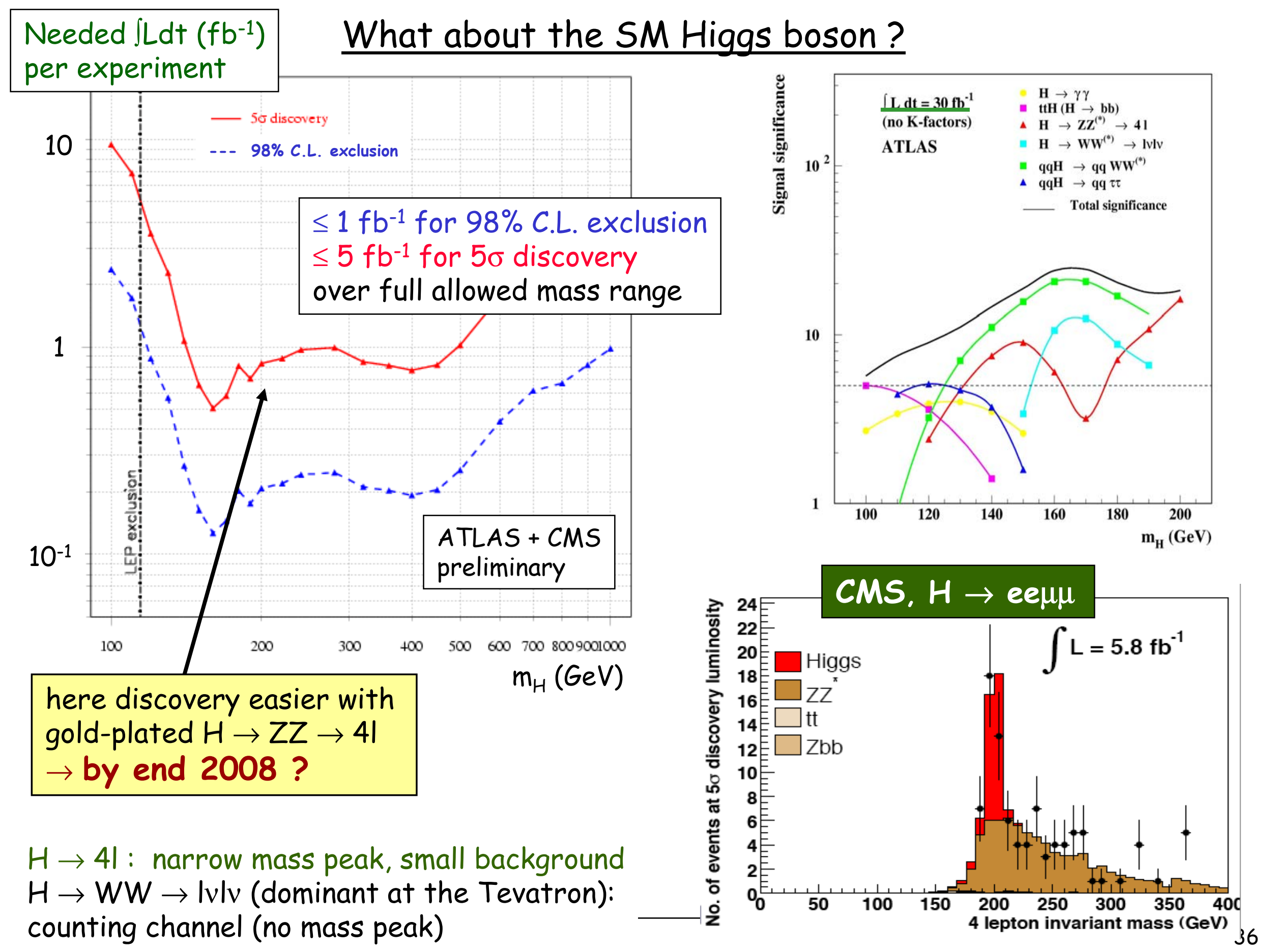


Light Higgs : more difficult ....

$m_{H} \sim 115 \mathrm{GeV} \quad 10 \mathrm{fb}^{-1}: S / \sqrt{ } \mathrm{B} \approx 4 \quad$ ATLAS

K-factors $\equiv \sigma(N L O) / \sigma(L O) \approx 2$

for $\mathrm{H} \rightarrow \gamma \gamma$ NOT included (conservative)

3 (complementary) channels with similar (small) significances:
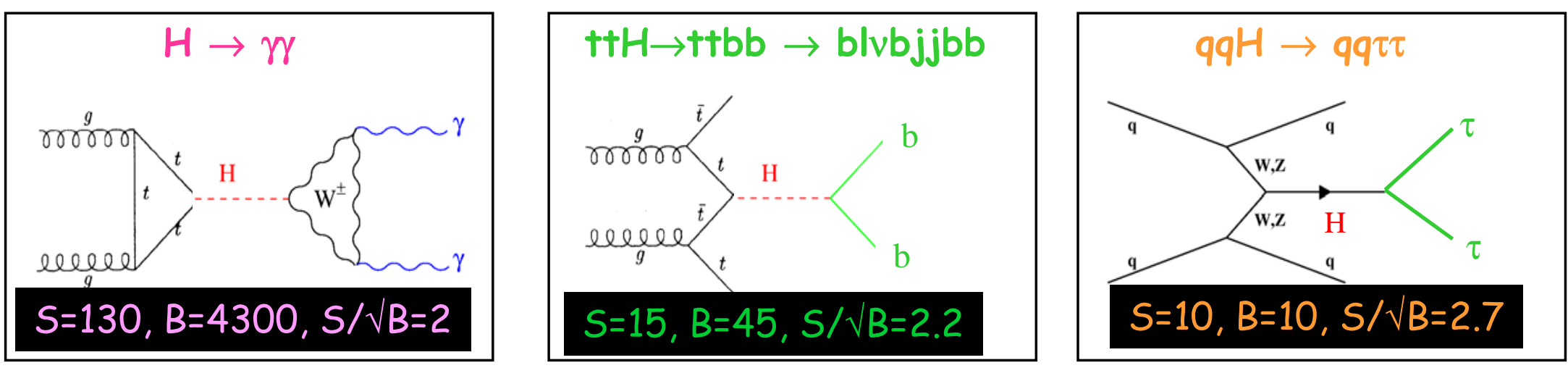

- different production and decay modes

- different backgrounds

- different detector/performance requirements:

-- ECAL crucial for $H \rightarrow \gamma$ (in particular response uniformity) $: \sigma / m \sim 1 \%$ needed

-- b-tagging crucial for $+\mathrm{H} H$ : 4 b-tagged jets needed to reduce combinatorics

-- efficient jet reconstruction over $|\eta|<5$ crucial for $q q H \rightarrow q q \tau \tau$ : forward jet tag and central jet veto needed against background

All three channels require very good understanding of detector performance and background control to $1-10 \% \rightarrow$ convincing evidence likely to come later than 2008

Note: $\mathrm{WH} \rightarrow \mathrm{lubb}$ (dominant at the Tevatron) provides less sensitivity than $\mathrm{t+H}$ at LHC

F. Gianotti, ICHEP06, Moscow, 02/08/2006 


\section{Those of you who have bet for ATLAS can still hope to make money out of it....}

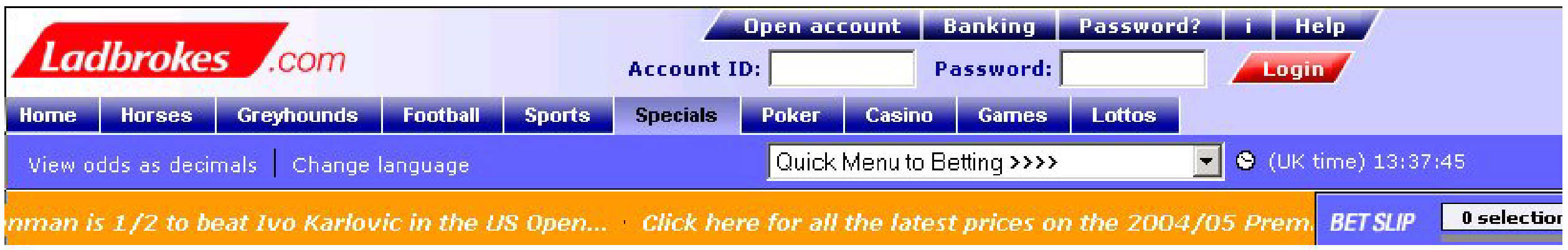

\begin{tabular}{|c|}
\hline SPECIALS \\
\hline 听) COMMENTARY \\
\hline$f$ REWARDS \\
\hline (i覂 CONTACT US \\
\hline $\begin{array}{l}\text { f BANKING METHODS } \\
\text { (i) RESPONSIBLE GAMBLING }\end{array}$ \\
\hline FEATURES \\
\hline
\end{tabular}

\section{SPECIALS}

Science Specials

ohmpic Specials

\section{POLITICS}

UK General Election

US Presidential Election

\section{AWARDS}

Booker Prize

MUSIC

Mercury Music Prize

FEATURES

HOSPITALITY

\section{SPECIALS}

Specials | Politics | Awards | Music

Specials - Science Specials

\begin{tabular}{|c|c|c|}
\hline Physics Breakthroughs & & \\
\hline $\begin{array}{l}\text { SELECTION } \\
\text { Click here or on ODDS to change wiew } \\
\text { order }\end{array}$ & ODDS & $\begin{array}{l}\text { EET NOW OF } \\
\text { ADD TO } \\
\text { MULTIPLE }\end{array}$ \\
\hline Understanding the origin of cosmic rays by 2010 & $4 / 1$ & Bet \\
\hline The ATLAS experiment at CERN finding the Higgs Boson by 2010 & $6 / 1$ & Bet \\
\hline The Laser Interferometer Gravitational Wave Observatory (LIGO) detecting gravitational waves by 2010 & $6 / 1$ & Bet \\
\hline Building a fusion power station by 2010 & $100 M$ & Bet \\
\hline
\end{tabular}

Selections will be settled on the basis of reports published in Hew Scientist magazine.

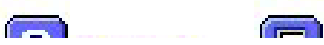




\section{Conclusions}

Impressive achievements in the machine construction over last months:

$>50 \%$ dipoles installed, problems with cryoline solved, better understanding of commissioning and operation, etc.

- New LHC schedule: - - machine and experiments closed 31 August 2007

- - commissioning run at $\sqrt{s}_{s}=900 \mathrm{GeV}$ end 2007

- - first physics run at $14 \mathrm{TeV}$ starting in Spring 2008

- Experiments (huge progress as well !) on track to meet above schedule. Test-beam and cosmics results indicate they work as expected.

- All efforts now to continue installation and commissioning of machine and detectors of unprecedented complexity, technology and performance

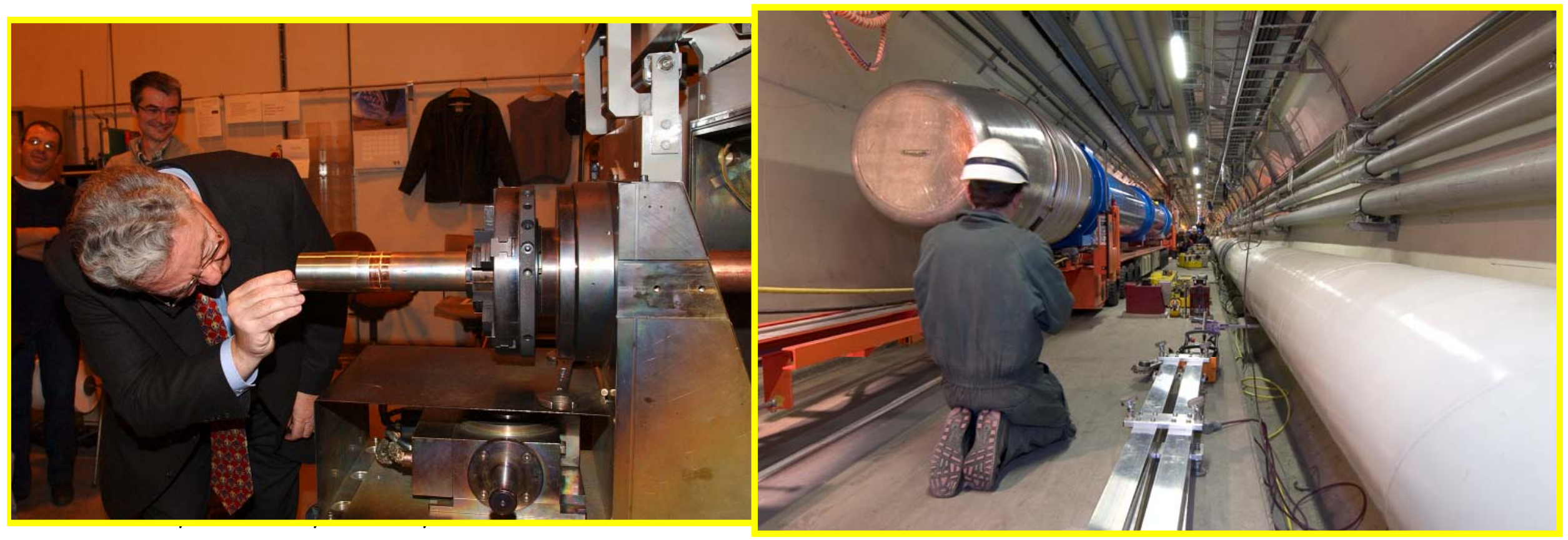


With the first collision data $\left(1-100 \mathrm{pb}^{-1}\right)$ at $14 \mathrm{TeV}$

Understand detector performance in situ in the LHC environment, and perform first physics measurements:

- Measure particle multiplicity in minimum bias (a few hours of data taking ...)

- Measure QCD jet cross-section to 30\%? (Expect $>10^{3}$ events with $E_{\mathrm{T}}(\mathrm{j})>1 \mathrm{TeV}$ with $100 \mathrm{pb}^{-1}$ )

- Measure W, Z cross-sections to $10 \%$ with $100 \mathrm{pb}^{-1}$ ?

- Observe a top signal with $30 \mathrm{pb}^{-1}$

- Measure tt cross-section to $20 \%$ and $m\left(\right.$ top) to $7-10 \mathrm{GeV}$ with $100 \mathrm{pb}^{-1}$ ?

- Improve knowledge of PDF (low-x gluons !) with W/Z with $O(100) \mathrm{pb}^{-1}$ ?

- First tuning of $M C$ (minimum-bias, underlying event, $t+, W / Z+j e t s, Q C D$ jets,...)

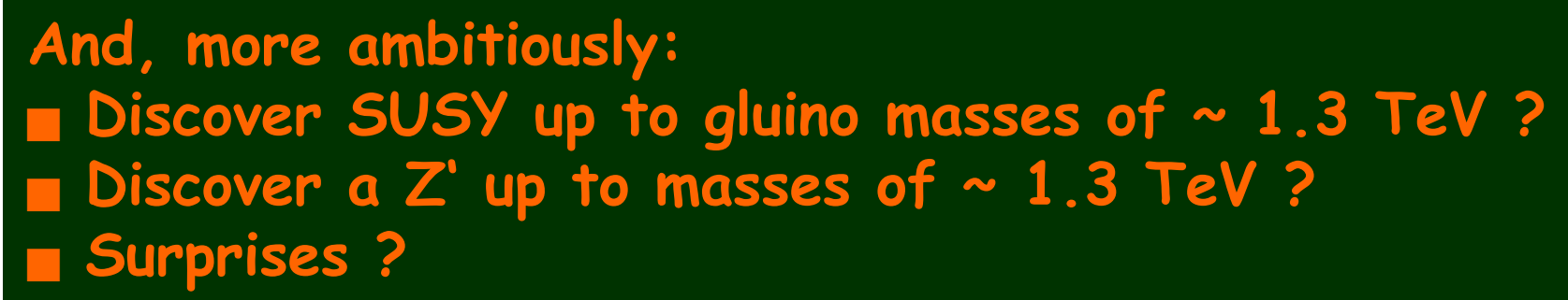


And, later on ....

The LHC will explore in detail the highly-motivated TeV-scale with a direct discovery potential up to $m \approx 5-6 \mathrm{TeV}$

$\rightarrow$ if New Physics is there, the LHC will find it

$\rightarrow$ it will say the final word about the SM Higgs mechanism and many TeV-scale predictions

$\rightarrow$ it may add crucial pieces to our knowledge of fundamental physics $\rightarrow$ impact also on astroparticle physics and cosmology

$\rightarrow$ most importantly: it will likely tell us which are the right questions to ask, and how to go on

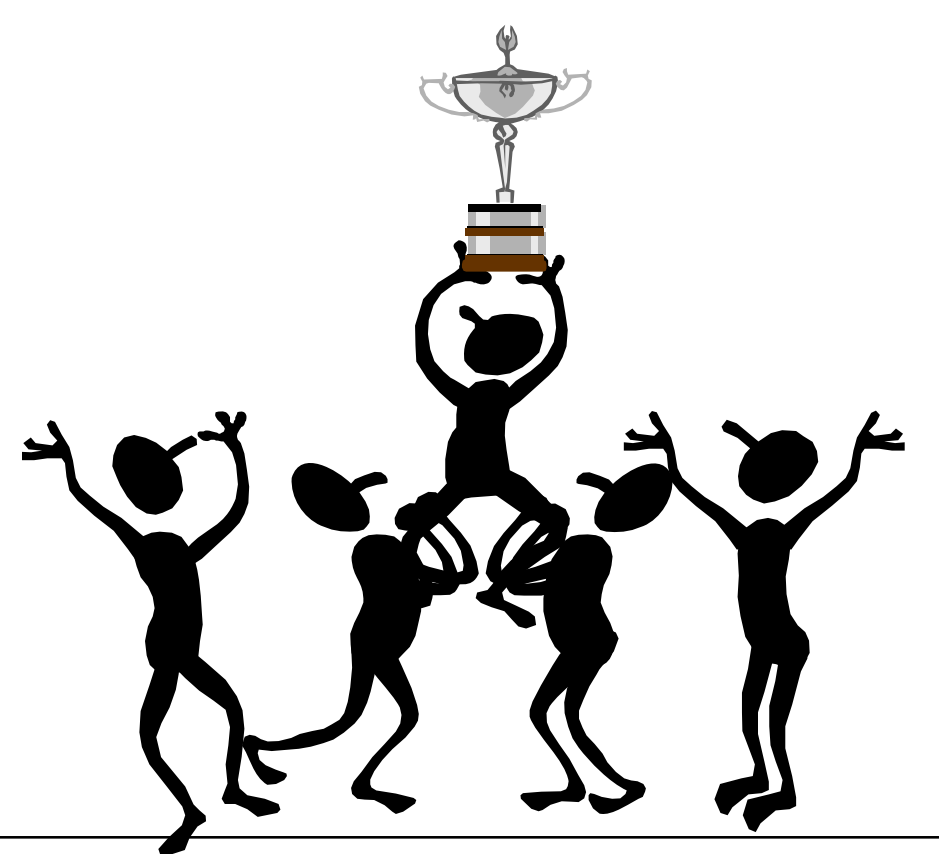




\section{Many thanks to:}

S.Asai, J.Baines, S.Bentvelsen, Ph.Bloch, M.Cirilli, M.-J.Costa, A.DeRoeck, L.Dobrzynski, M.Elsing, J.Engelen, L.Evans, D.Fournier, E.Gallo, W. Hulsbergen, P.Jenni, N.Kanaya, M.Martinez, A.Moraes, T.Nakada, M.Nessi, H.Okawa, G.Polesello, R.Saban, W.Scandale, M.Smizanska, S. Stapnes, G.Unal, W.Verkerke, T.Virdee, and many others ... 


\section{Back-up slides}




\section{What about the comparison with the Tevatron?}

\section{$L\left(\mathbf{f b}^{-1}\right)$ /exp.}

100 - Higgs Sensitivity Study ('03)

statistical power only

(no systematics)

10

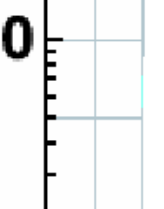

1

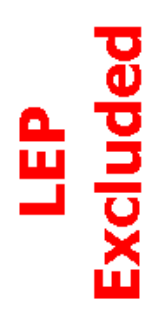

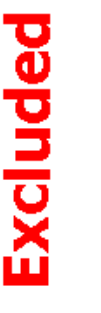

PRELIMINARY
$C D F+D O$ sensitivity

SUSY/Higgs Workshop

('98-'99)

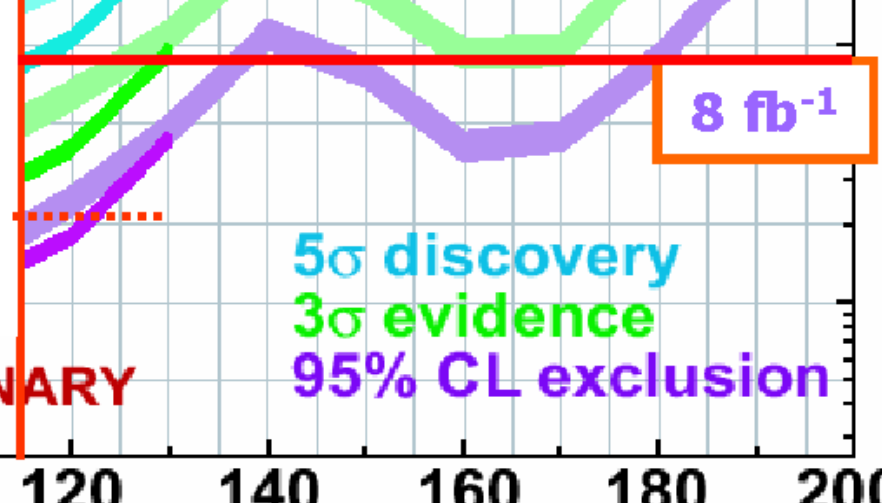

$\mathrm{m}_{\mathrm{H}}(\mathrm{GeV})$
Today : $1.3 \mathrm{fb}^{-1}$ /exp. on tape Projections for end 2009:

$4 \mathrm{fb}^{-1}$ : present machine performance $8 \mathrm{fb}^{-1}$ : electron cooling of pbar and other improvements

\section{With 4 (8) $\mathrm{fb}^{-1}$ :}

no $5 \sigma$ sensitivity

$3 \sigma$ evidence up to 120 (130) GeV

$95 \%$ C.L. exclusion up to 130 (180) GeV

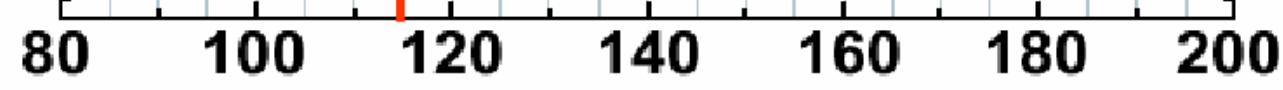

competition between Tevatron and $\mathrm{LHC}$ in 2008-2009 if $m_{H}<190 \mathrm{GeV}$ ?

\begin{tabular}{|c|c|c|}
\hline $\begin{array}{l}\text { Tevatron vs } L H C \\
\text { after kin. cuts }\end{array}$ & $\begin{array}{l}\mathrm{WH} \rightarrow \mathrm{l} v \mathrm{bb} \\
\left(\mathrm{m}_{\mathrm{H}}=120 \mathrm{GeV}\right)\end{array}$ & $\begin{array}{c}\mathrm{H} \rightarrow \mathrm{WW}(*) \\
\left(\mathrm{m}_{\mathrm{H}}=160 \mathrm{GeV}\right)\end{array}$ \\
\hline $\begin{array}{l}\mathrm{S} \quad(14 \mathrm{TeV} / 2 \mathrm{TeV}) \\
\mathrm{B} \quad(14 \mathrm{TeV} / 2 \mathrm{TeV}) \\
\mathrm{S} / \mathrm{B}(14 \mathrm{TeV} / 2 \mathrm{TeV}) \\
\mathrm{S} / \sqrt{\mathrm{B}}(14 \mathrm{TeV} / 2 \mathrm{TeV})\end{array}$ & $\begin{array}{l}\approx 5 \\
\approx 25 \\
\approx 0.2 \\
\approx 1\end{array}$ & $\begin{array}{l}\approx 17 \\
\approx 6 \\
\approx 3 \\
\approx 7\end{array}$ \\
\hline
\end{tabular}

Assuming same integrated luminosity and same detector performance at Tevatron and LHC 


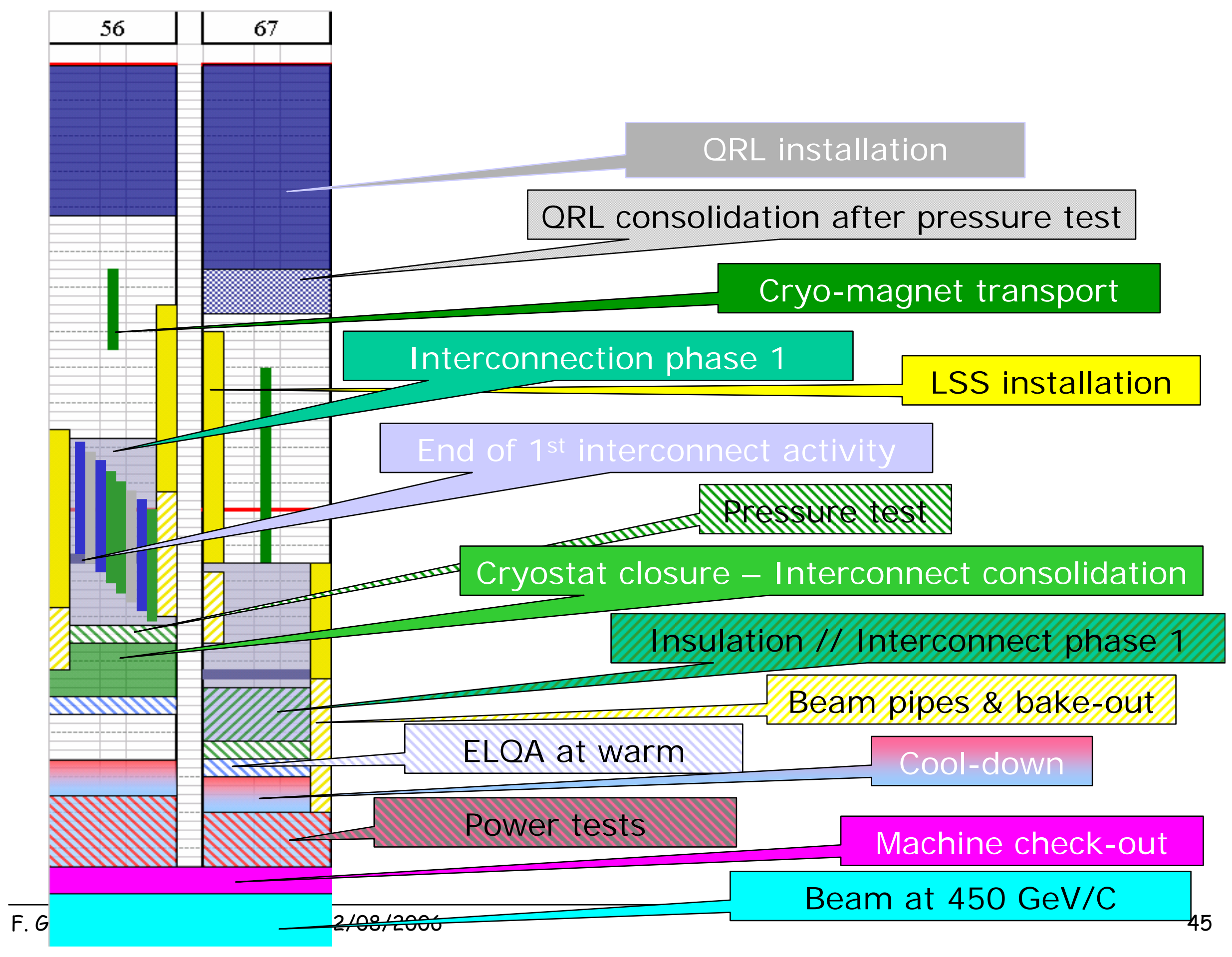




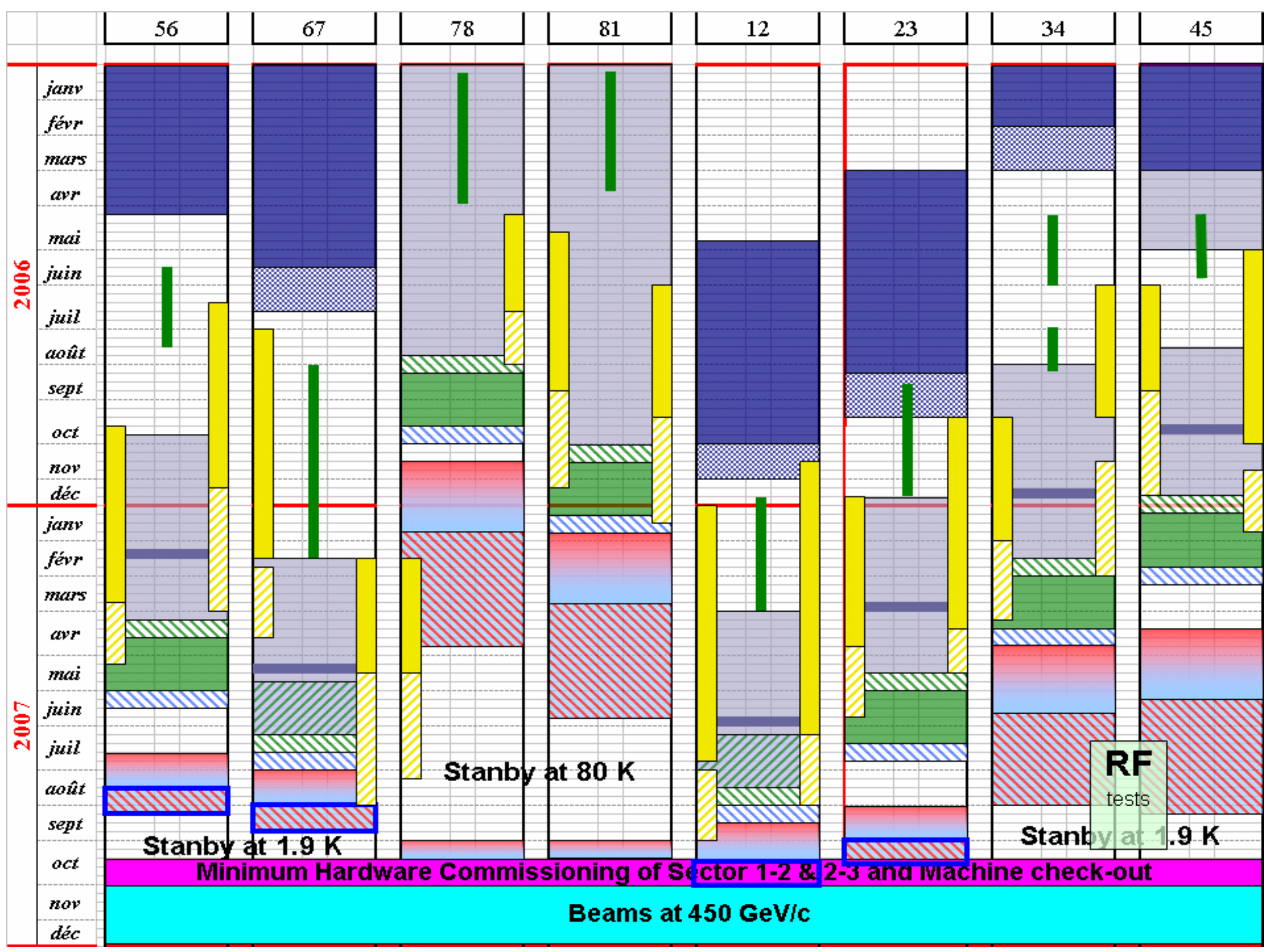




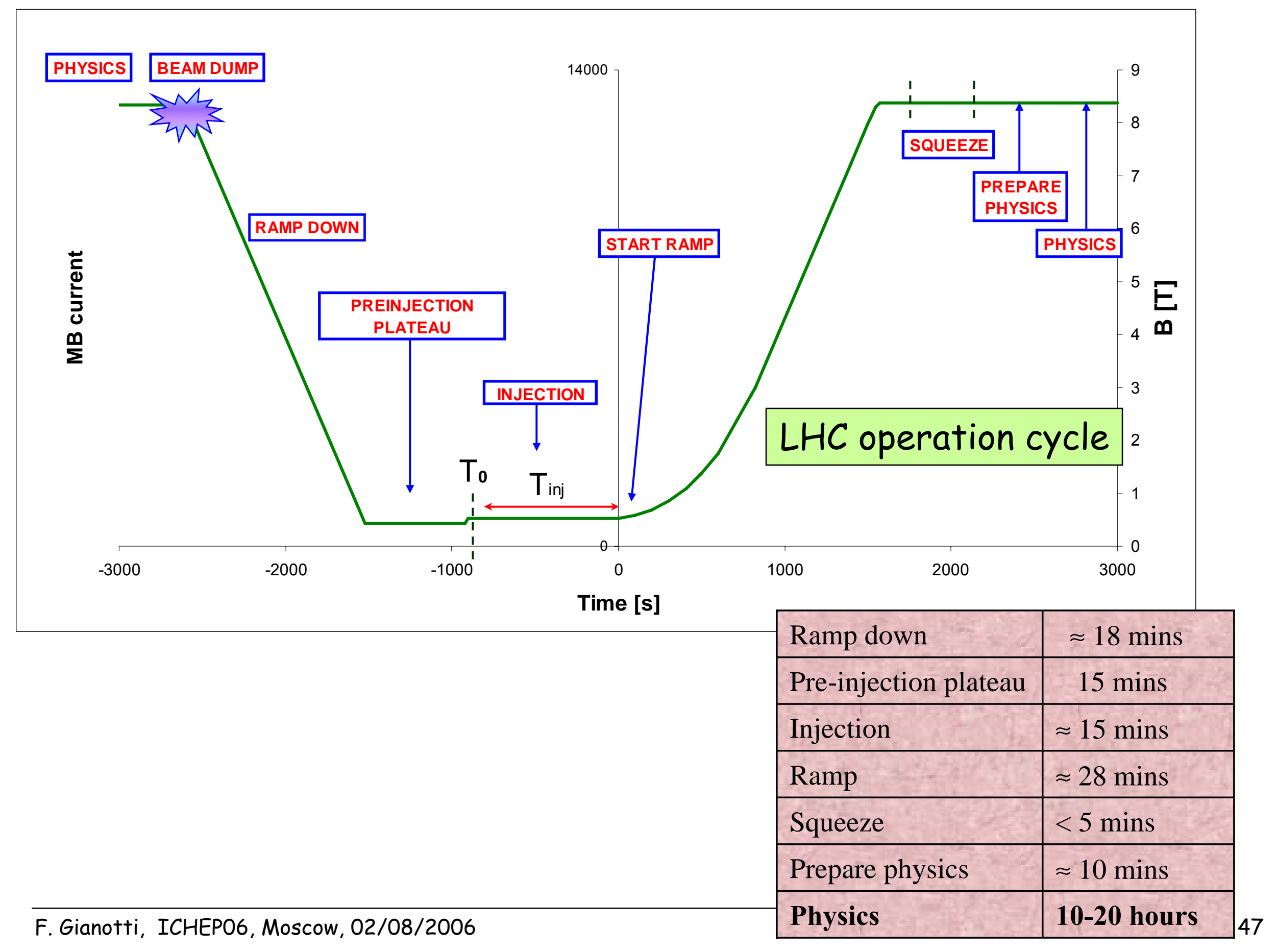




\section{Distribution Feed Box}

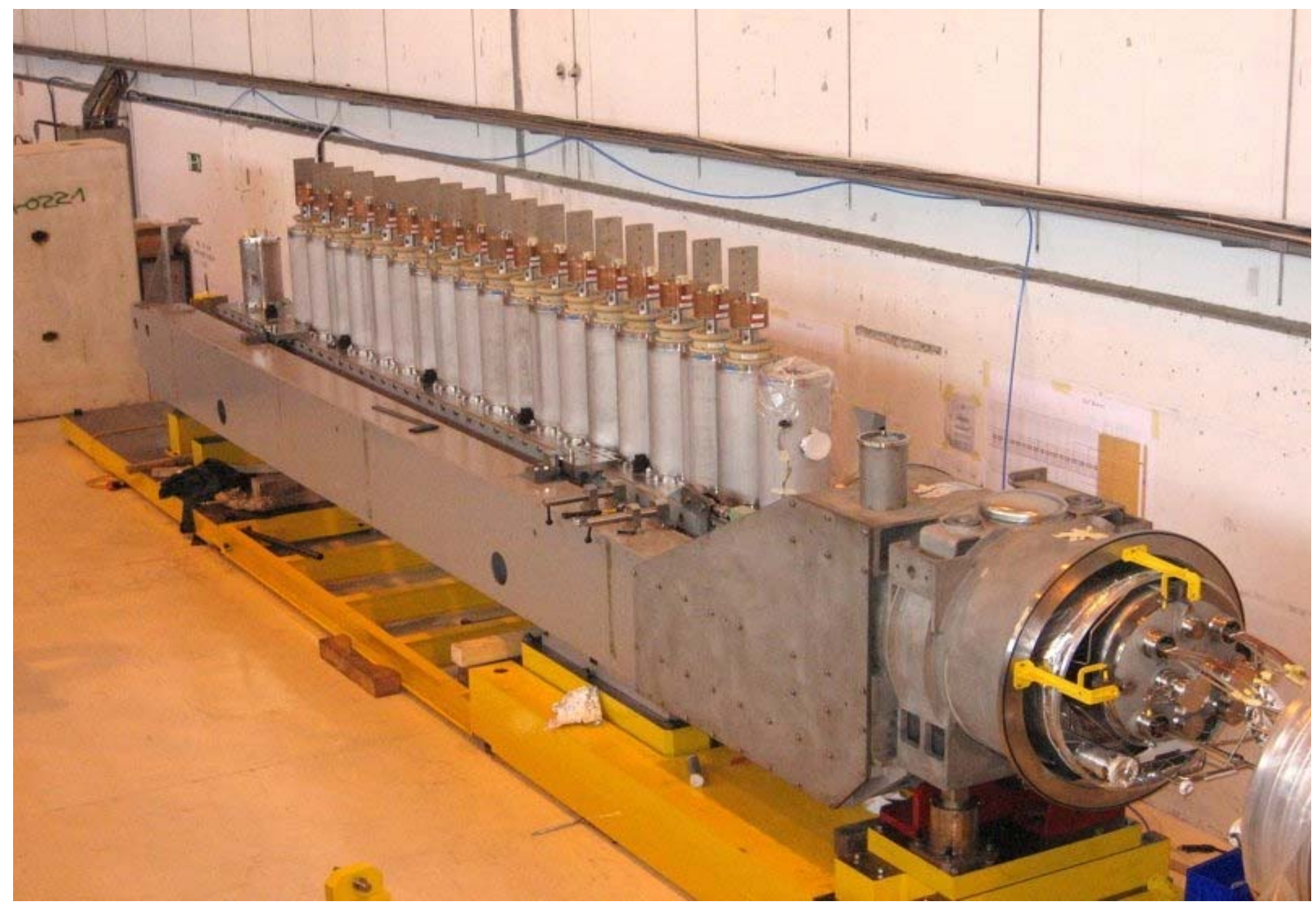




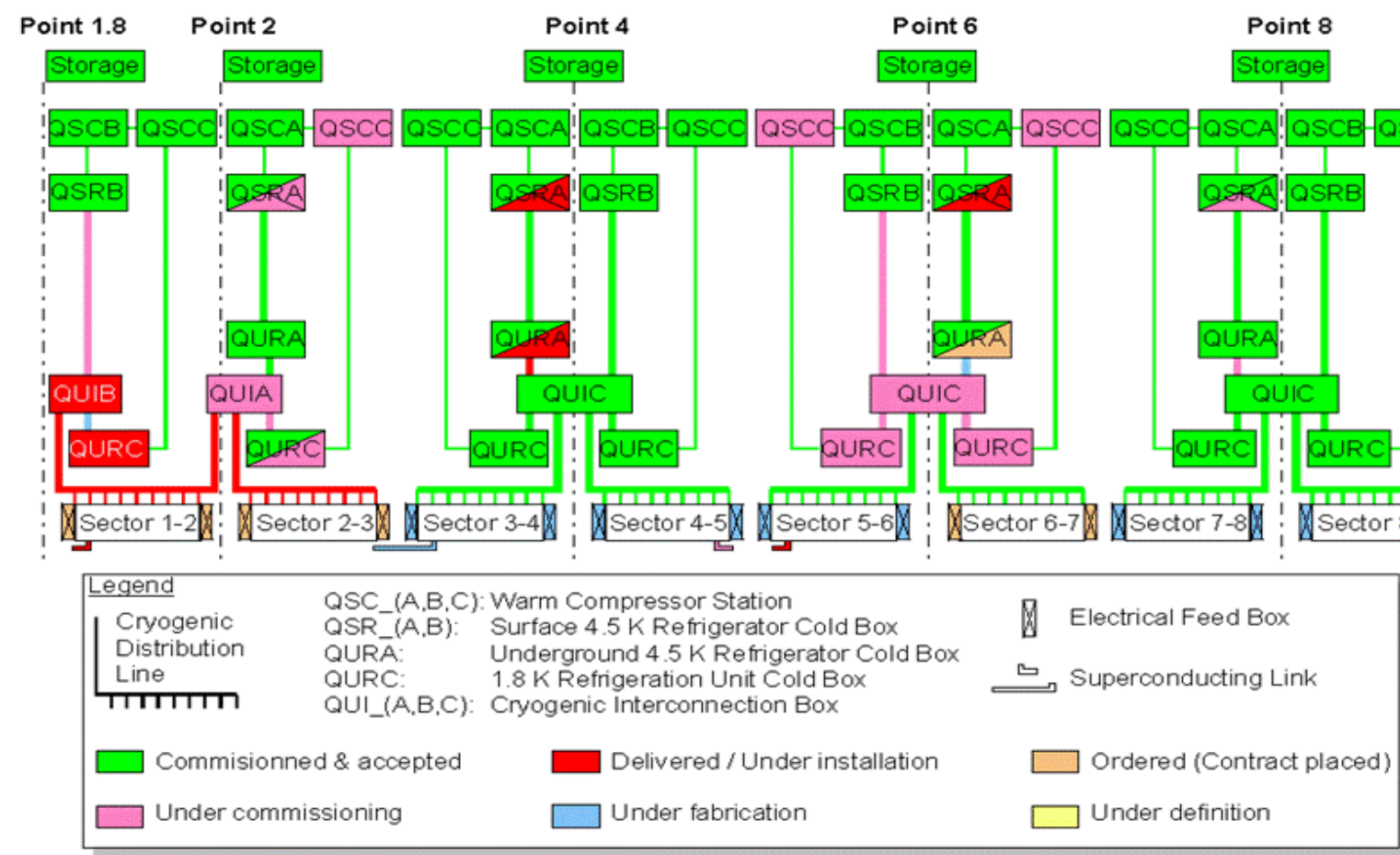

Updated 31 May 2006 Data provided by L. Tavian AT-ACR 


\section{Staged commissioning plan for protons}

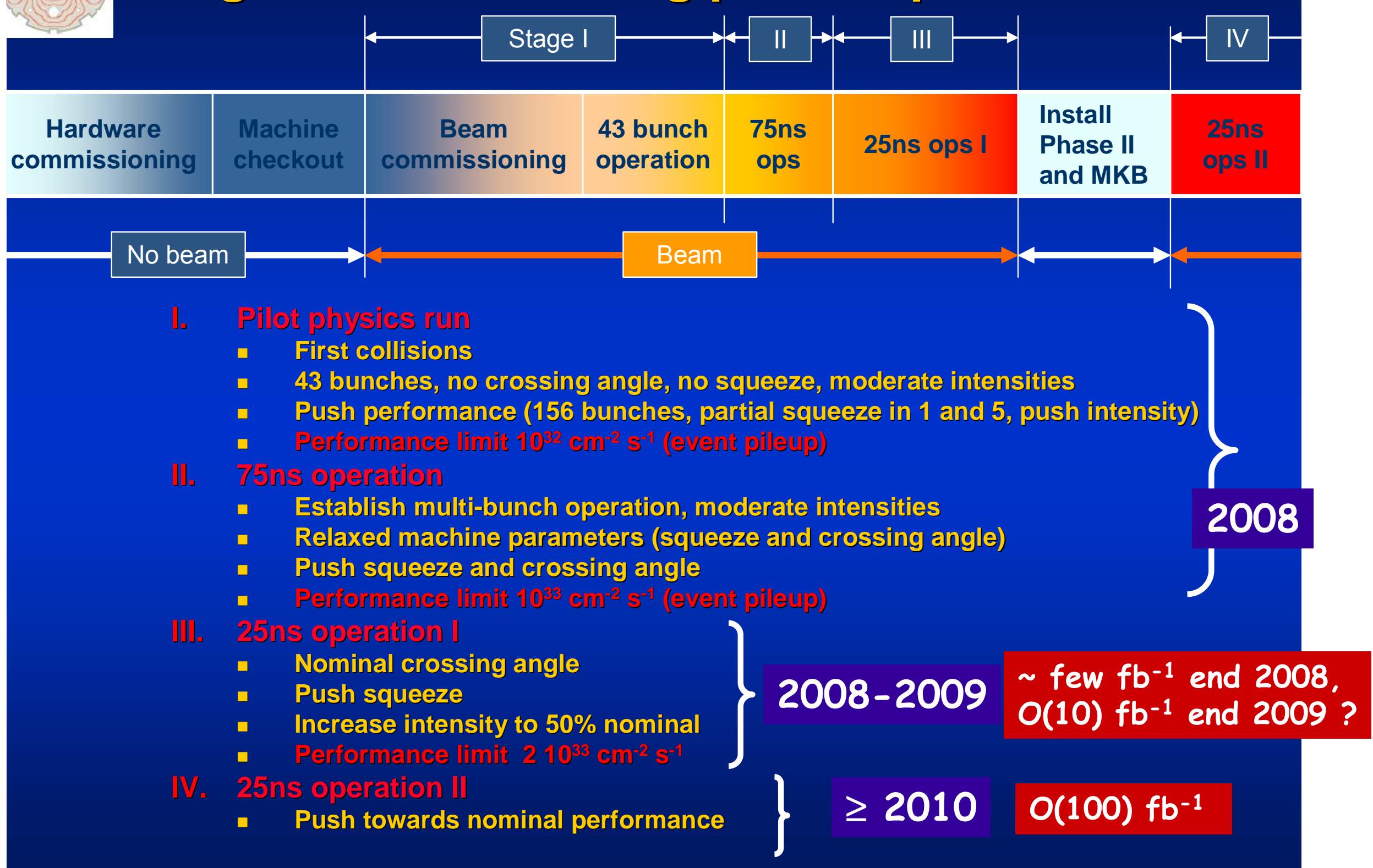

Note: dates and integrated luminosities are MY interpretation 


\begin{tabular}{|c|c|c|}
\hline & ATLAS & CMS \\
\hline MAGNET (S) & $\begin{array}{l}\text { Air-core toroids }+ \text { solenoid in inner cavity } \\
4 \text { magnets } \\
\text { Calorimeters in field-free region }\end{array}$ & $\begin{array}{l}\text { Solenoid } \\
\text { Only } 1 \text { magnet } \\
\text { Calorimeters inside field }\end{array}$ \\
\hline TRACKER & $\begin{array}{l}\text { Si pixels }+ \text { strips } \\
\mathrm{TRT} \rightarrow \text { particle identification } \\
\mathrm{B}=2 \mathrm{~T} \\
\sigma / \mathrm{p}_{\mathrm{T}} \sim 5 \times 10^{-4} \mathrm{p}_{\mathrm{T}} \oplus 0.01\end{array}$ & $\begin{array}{l}\text { Si pixels + strips } \\
\text { No particle identification } \\
\mathrm{B}=4 \mathrm{~T} \\
\sigma / \mathrm{p}_{\mathrm{T}} \sim 1.5 \times 10^{-4} \mathrm{p}_{\mathrm{T}} \oplus 0.005\end{array}$ \\
\hline EM CALO & $\begin{array}{l}\text { Pb-liquid argon } \\
\sigma / \mathrm{E} \sim 10 \% / \sqrt{\mathrm{E}} \quad \text { uniform } \\
\text { longitudinal segmentation }\end{array}$ & $\begin{array}{l}\mathrm{PbWO}_{4} \text { crystals } \\
\sigma / \mathrm{E} \sim 2-5 \% / \sqrt{\mathrm{E}} \\
\text { no longitudinal segm. }\end{array}$ \\
\hline HAD CALO & $\begin{array}{l}\text { Fe-scint. + Cu-liquid argon }(10 \lambda) \\
\sigma / \mathrm{E} \sim 50 \% / \sqrt{ } \mathrm{E} \oplus 0.03\end{array}$ & $\begin{array}{l}\text { Cu-scint. (> } 5.8 \lambda+\text { catcher }) \\
\sigma / \mathrm{E} \sim 100 \% / \sqrt{ } \mathrm{E} \oplus 0.05\end{array}$ \\
\hline MUON & $\begin{array}{l}\text { Air } \rightarrow \sigma / \mathrm{p}_{\mathrm{T}} \sim 7 \% \text { at } 1 \mathrm{TeV} \\
\text { standalone }\end{array}$ & $\begin{array}{l}\mathrm{Fe} \rightarrow \sigma / \mathrm{p}_{\mathrm{T}} \sim 5 \% \text { at } 1 \mathrm{TeV} \\
\text { combining with tracker }\end{array}$ \\
\hline
\end{tabular}




\section{CMS Magnet Test and Cosmic Challenge (MTCC)}

Ramping up of the magnet to nominal field started. A combined test of a slice of CMS will then be performed with cosmics.

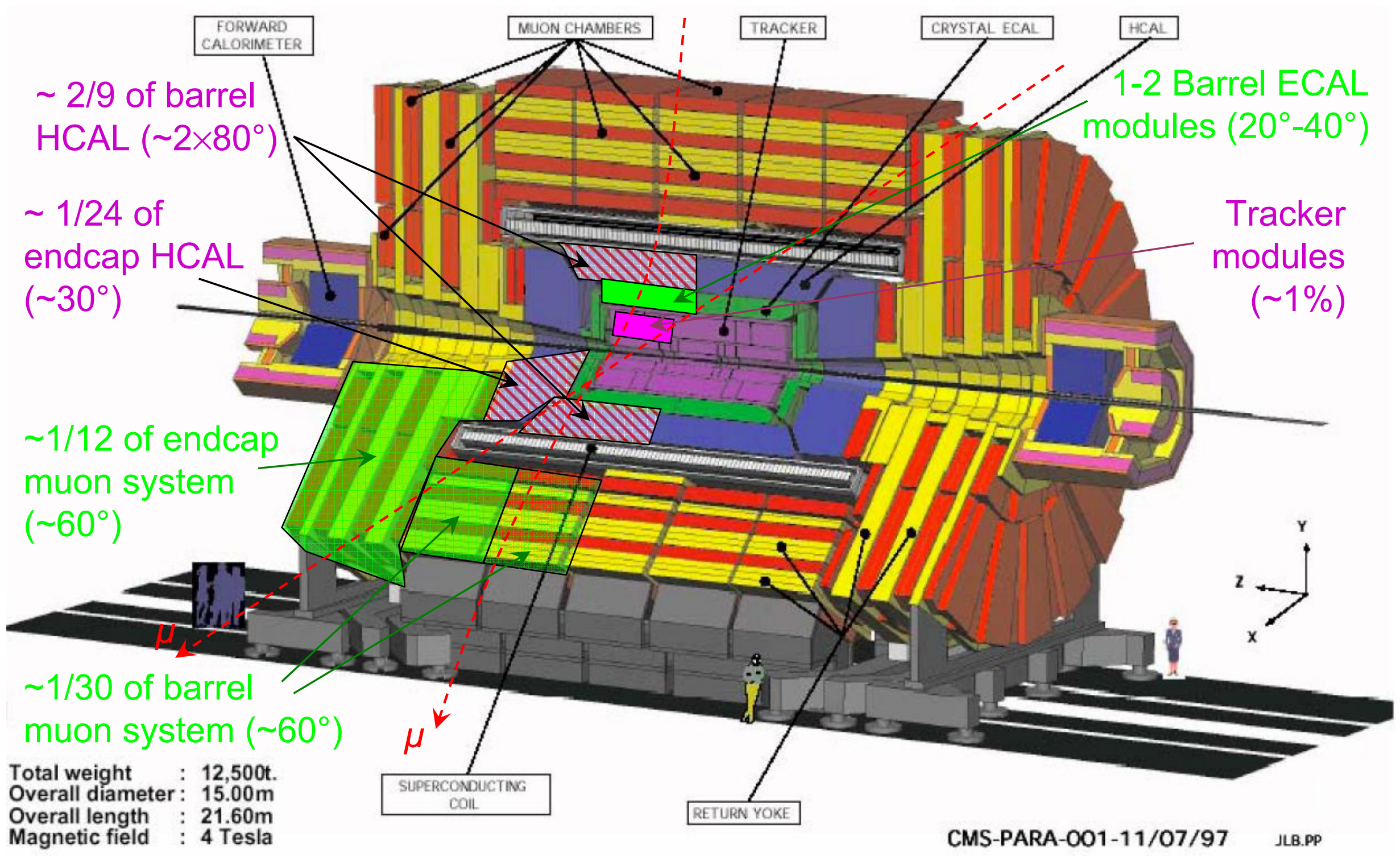




\section{Commissioning ECAL with cosmics (first studies ...}

- check calorimeter timing to $<1$ ns --> input to optimal filtering in electronics

- check calorimeter position in $\eta / \varphi$ wrt other sub-detectors to $<1 \mathrm{~mm}$

- check response uniformity vs $\eta: \approx 0.5 \%$ precision could be achieved

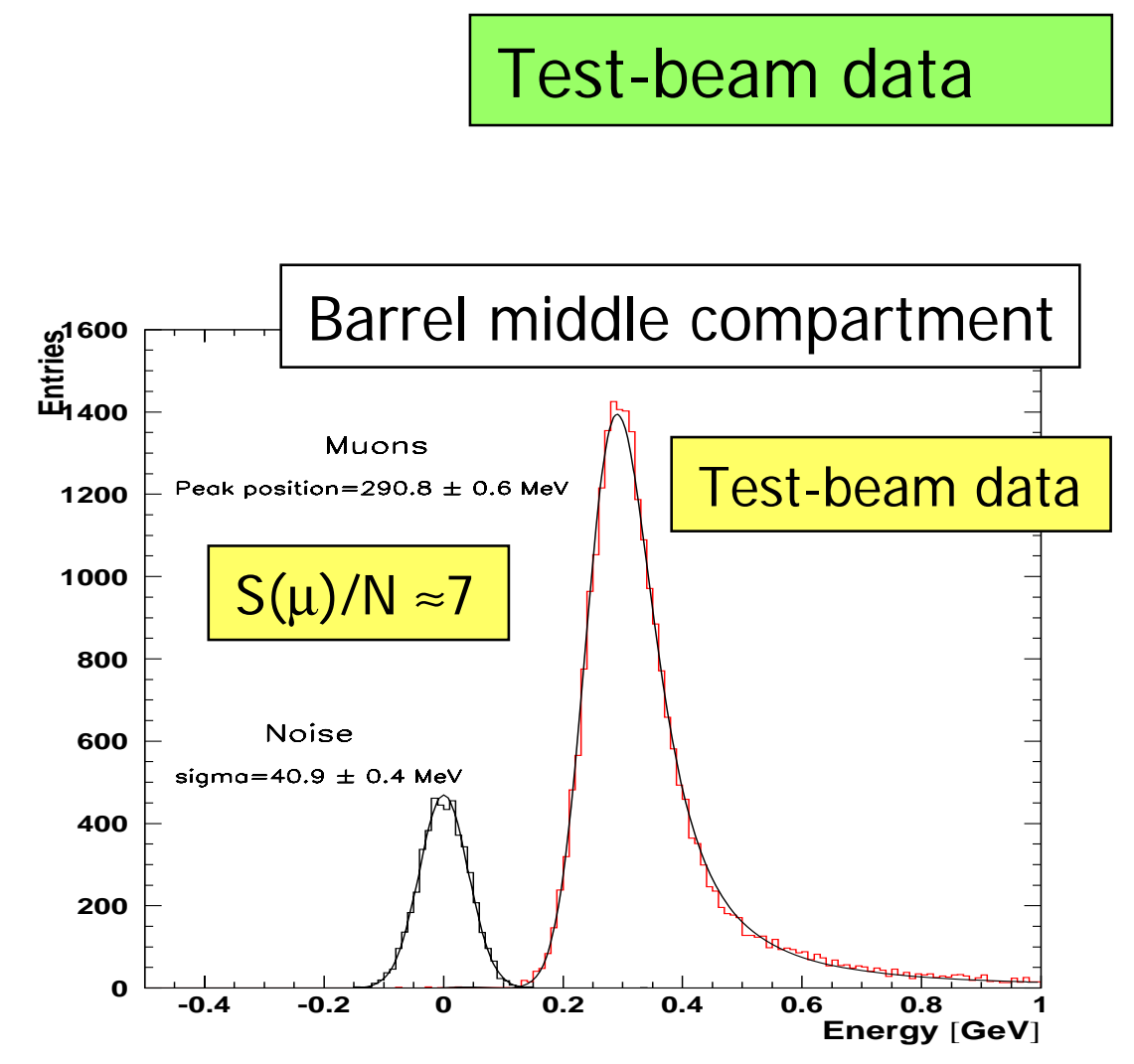

F. Gianotti, ICHEP06, Moscow, 02/08/2006

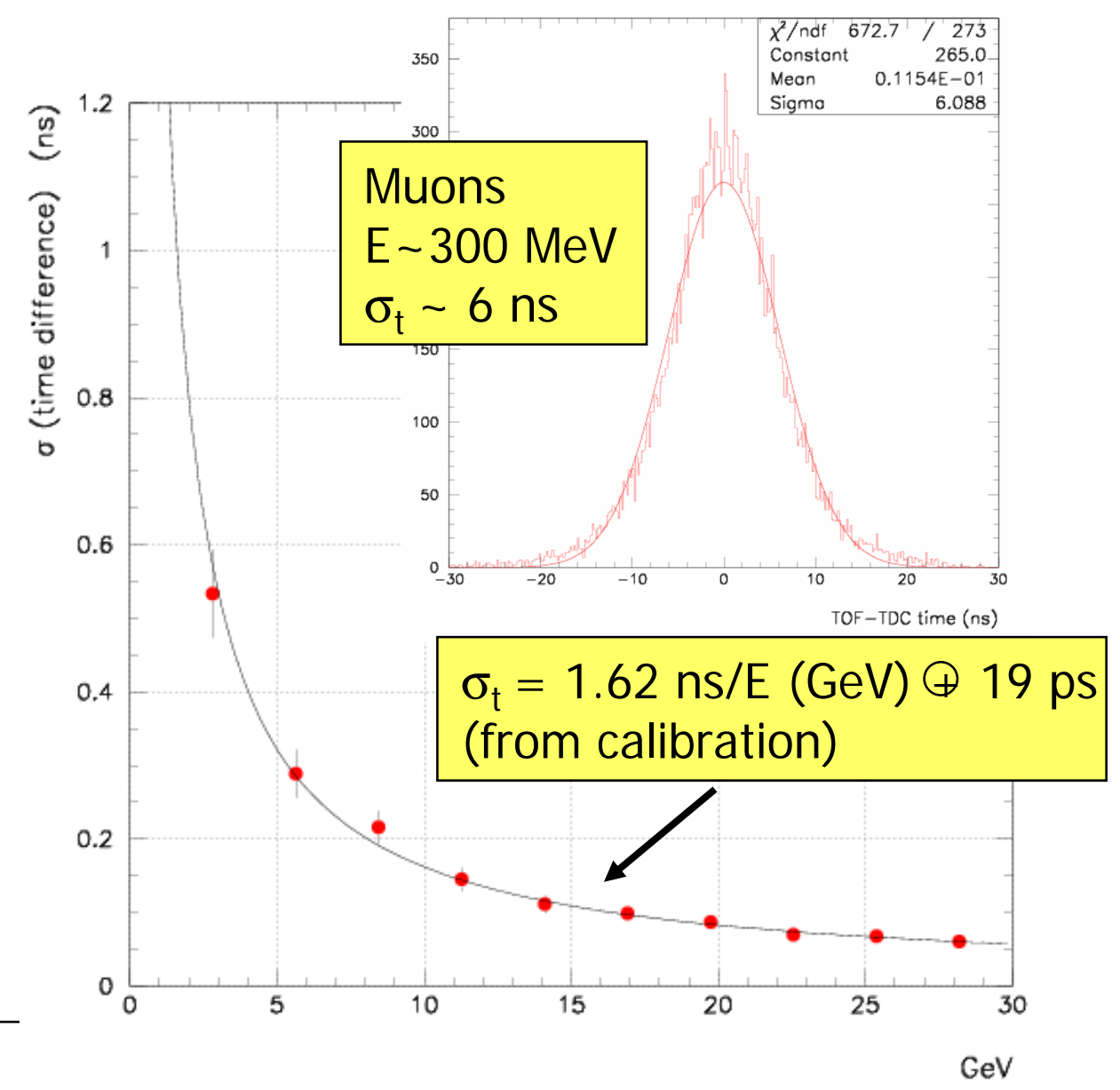




\section{Commissioning ID with cosmics and beam gas (some ideas.}

Cosmics: $\mathrm{O}(\mathrm{HHz})$ tracks in Pixels+SCT+TRT

- useful statistics for debugging readout, maps of dead modules, etc.

- check relative position Pixels/SCT/TRT and of ID wrt ECAL and Muon Spectrometer

- first alignment: may achieve statistical precision of $\sim 10 \mu \mathrm{m}$ in parts of Pixels/SCT, $50 \mu \mathrm{m}$ in TRT

- first calibration of $t_{0}$ and $R$ - $t$ relation in straws

\section{Beam-gas:}

- $25 \mathrm{~Hz}$ of reconstructed tracks with $\mathrm{p}_{\mathrm{T}}>1 \mathrm{GeV}$ and $|\mathrm{z}|<20 \mathrm{~cm}$ $>10^{7}$ tracks (similar to LHC events) in 2 months

- enough statistics for alignment in "relaxed" environment --> exceed initial survey precision of $\sim 100 \mu \mathrm{m}$

F. Gianotti, ICHEPO6, Moscow, 02/08/2006

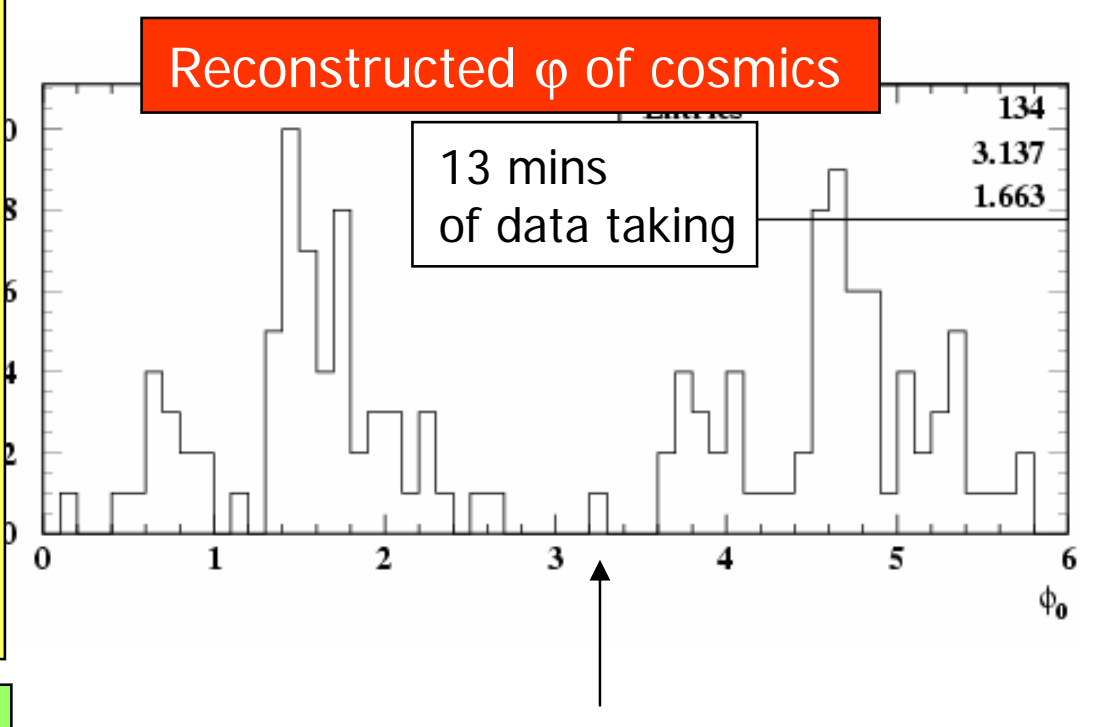

standard ATLAS patt. rec. (no optimisation for cosmics ...) 
A few examples and educated guesses based on detector construction quality, test-beam results, cosmics, and simulation studies

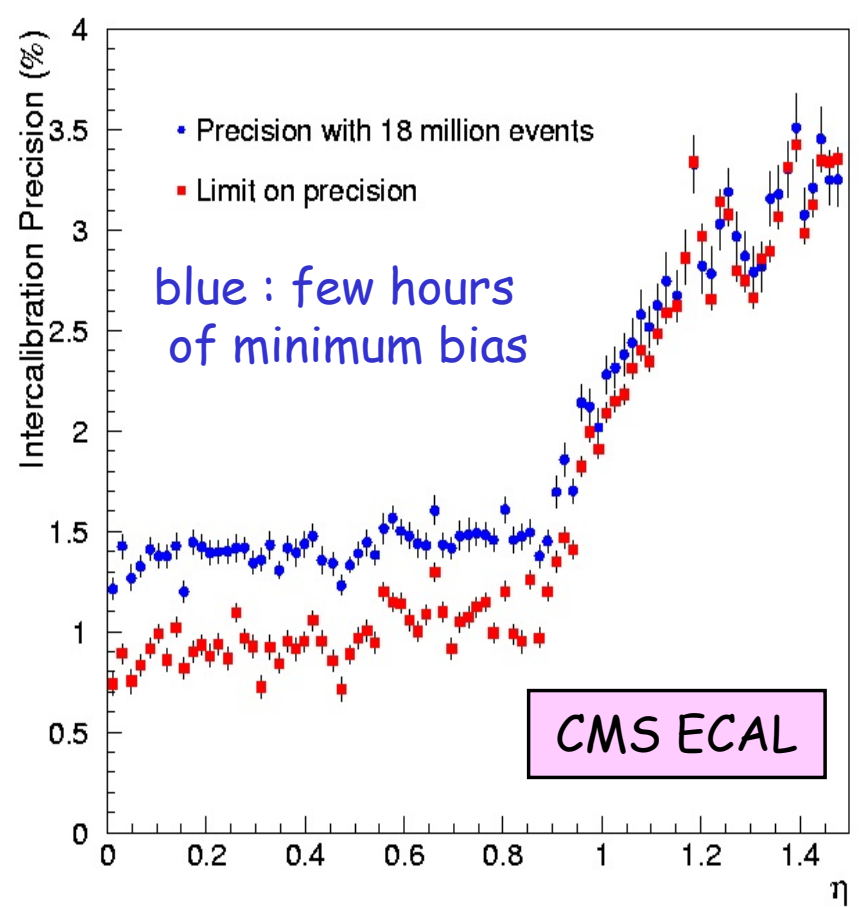

\begin{tabular}{|c|c|c|}
\hline & Expected performance day 1 & Physics samples to improve (examples) \\
\hline $\begin{array}{ll}\text { ECAL } & \text { uniformity } \\
e / \gamma & \text { scale }\end{array}$ & $\begin{array}{l}\sim 1 \% \text { (ATLAS), 3\% (CMS) } \\
\sim 2 \%\end{array}$ & $\begin{array}{l}\text { Minimum-bias, } Z \rightarrow e e \\
Z \rightarrow e e\end{array}$ \\
\hline $\begin{array}{l}\text { HCAL uniformity } \\
\text { Jet scale }\end{array}$ & $\begin{array}{l}\sim 3 \% \\
<10 \%\end{array}$ & $\begin{array}{l}\text { Single pions, } Q C D \text { jets } \\
Z(\rightarrow I I)+1 j, W \rightarrow j j \text { in tt events }\end{array}$ \\
\hline Tracking alignment & $20-200 \mu \mathrm{m}$ in $\mathrm{R \phi} ?$ & Generic tracks, isolated $\mu, Z \rightarrow \mu \mu$ \\
\hline
\end{tabular}

Ultimate statistical precision achievable after few weeks of operation. Then face systematics. E.g. : tracker alignment : $100 \mu \mathrm{m}(1$ month $) \rightarrow 10 \mu \mathrm{m}$ ( 6 months $) \rightarrow 5 \mu \mathrm{m}$ (1 year) ? 
Huge (QCD) backgrounds (consequence of high energy ...)

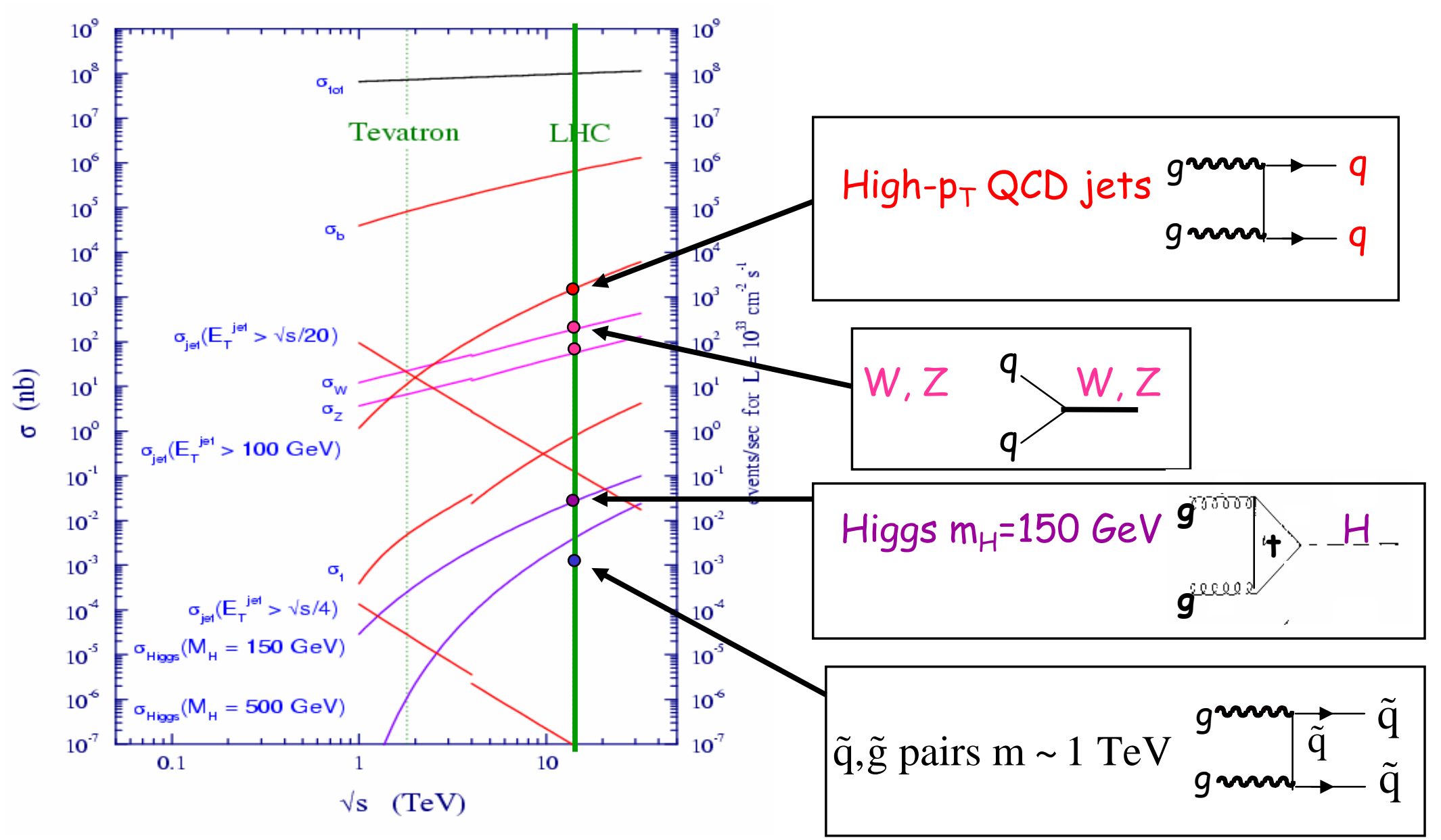

- No hope to observe light objects $(W, Z, H$ ?) in fully-hadronic final states $\rightarrow$ rely on $I, \gamma$

- Fully-hadronic final states (e.g. $q^{\star} \rightarrow q 9$ ) can be extracted from backgrounds only with hard $\mathrm{O}(100 \mathrm{GeV}) \mathrm{p}_{\mathrm{T}}$ cuts $\rightarrow$ works only for heavy objects

- Mass resolutions of 1\% (10\%) needed for I, $\gamma$ (jets) to extract tiny signals from backgrounds, and excellent particle identification (e.g. e/jet separation)

- $S(E W) / B(Q C D)$ larger at Tevatron than at LHC

F. Gianotti, ICHEP06, Moscow, 02/08/2006 
How many events per experiment at the beginning?

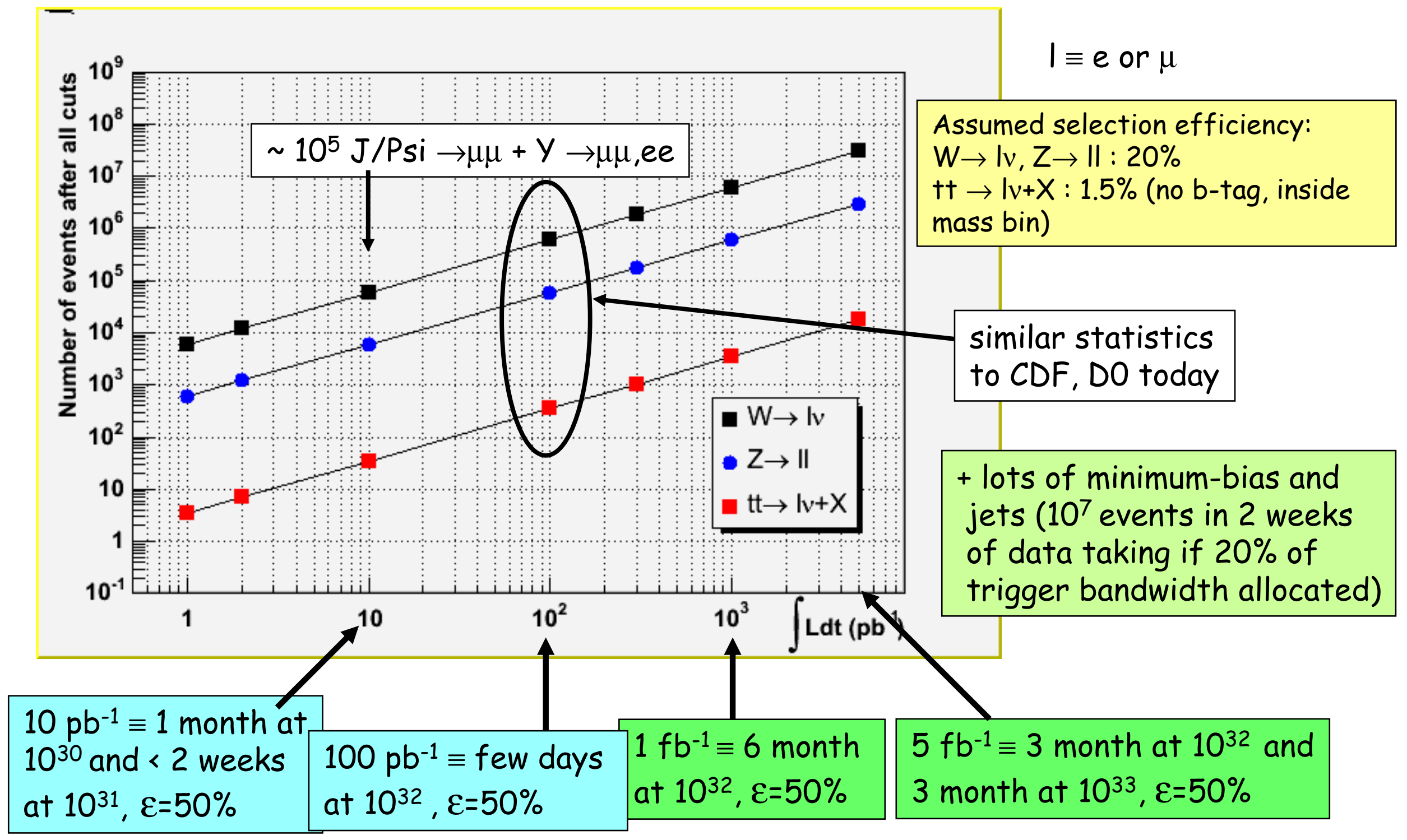

F. Gianotti, ICHEP06, Moscow, 02/08/2006 
W, Z cross-sections: to $3-4 \%$

(NNLO calculation $\rightarrow$ dominated by PDF)

tt cross-section to $\sim 7 \%$ (NLO+PDF)

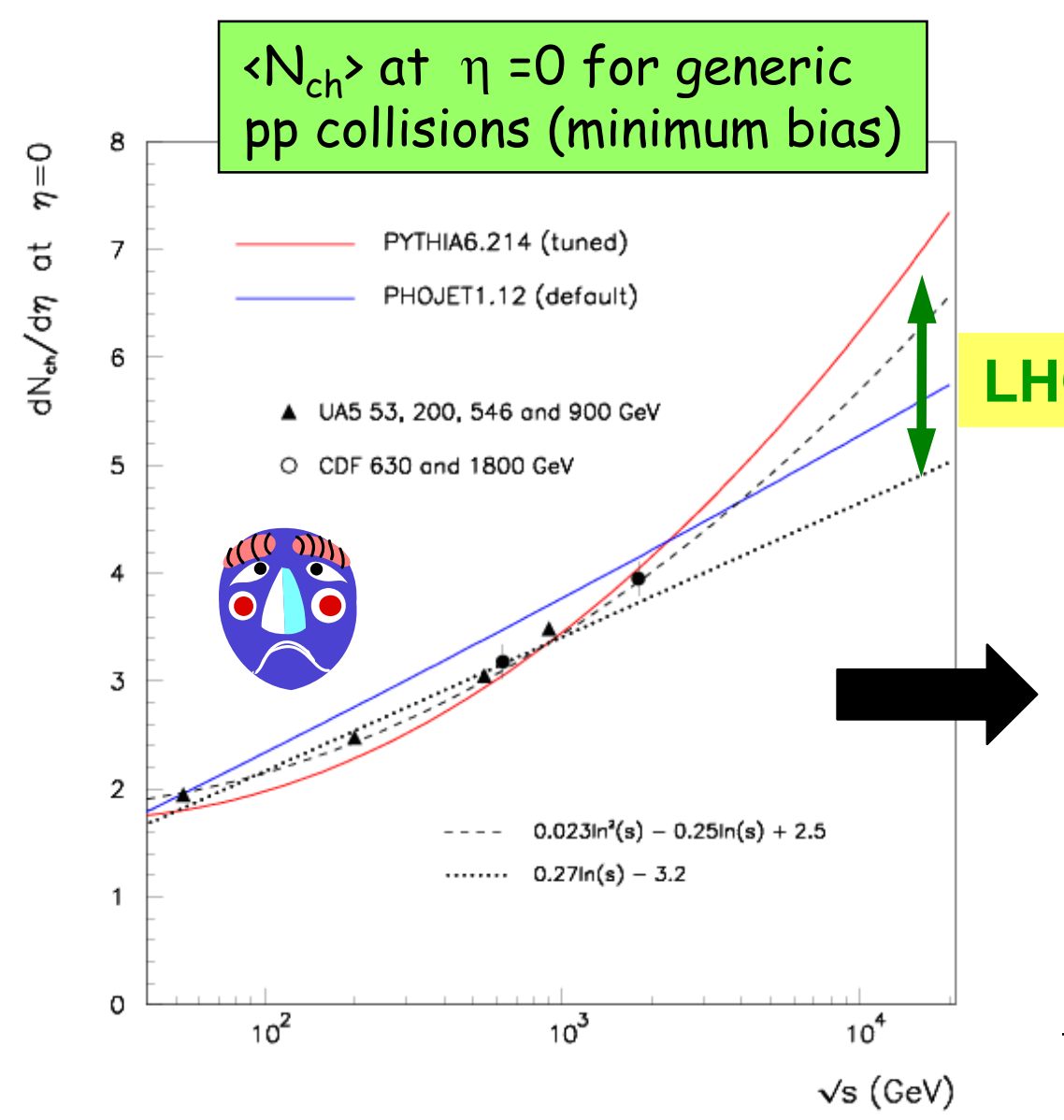

Lot of progress with NLO matrix element $M C$ interfaced to parton shower $M C$ (MC@ NLO, AlpGen,.. )

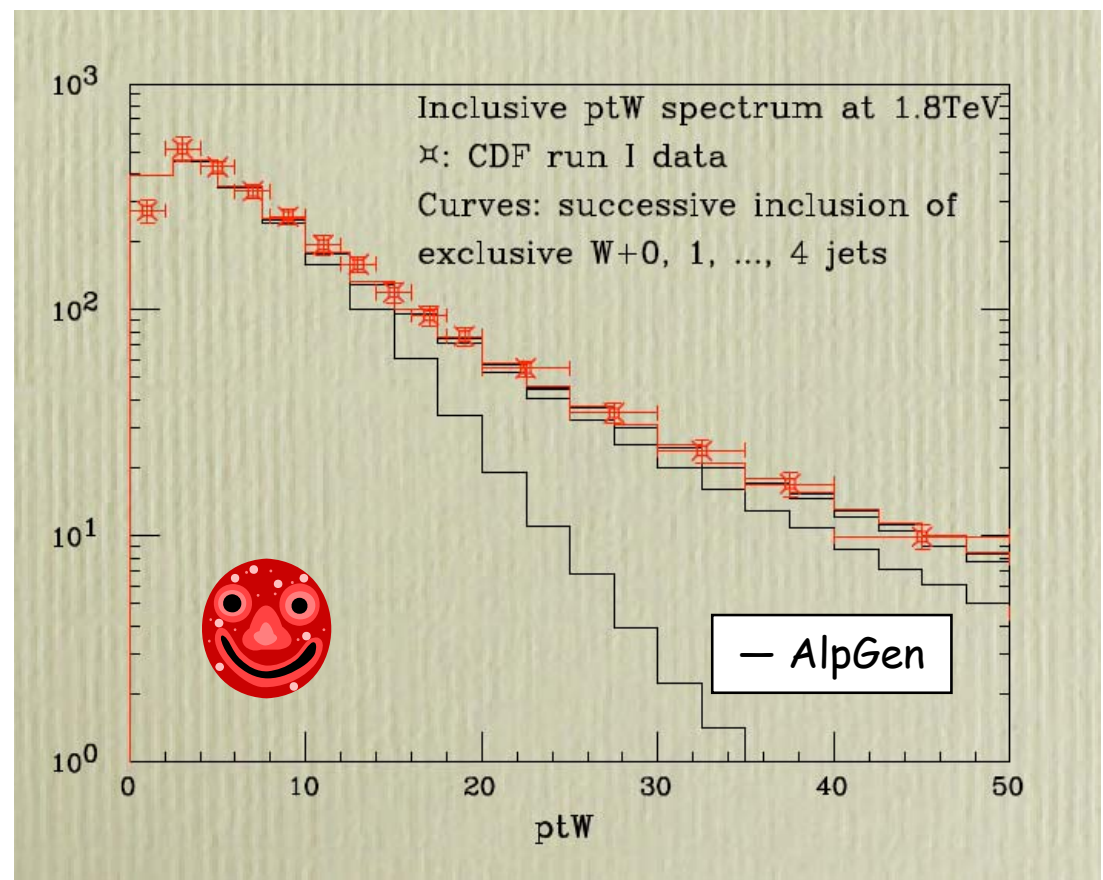

Candidate to very early measurement:

few $10^{4}$ events enough to get $d N_{c h} / d \eta, d N_{c h} / d p_{T}$

$\rightarrow$ tuning of MC models

$\rightarrow$ understand basics of pp collisions, occupancy, pile-up, ... 


\section{B-physics with 100pb-1 - statistics in dominant inclusive, exclusive channels}

\begin{tabular}{|l|l|l|}
\hline & 10 pb-1 & 100 pb-1 \\
\hline$p p \rightarrow \mu 6 X$ & $60.10^{6}$ & $600.10^{6}$ \\
\hline$b b \rightarrow \mu 6 X$ & $40.10^{6}$ & $400.10^{6}$ \\
\hline$c c \rightarrow \mu 6 X$ & $20.10^{6}$ & $200.10^{6}$ \\
\hline$b b \rightarrow \mu 6 \mu 3 X$ & $2.10^{6}$ & $20.10^{6}$ \\
\hline$p p \rightarrow J / \psi(\mu 6 \mu 3)$ & $2.810^{5}$ & $2.810^{6}$ \\
\hline$\Upsilon(\mu 6 \mu 3)$ & $0.9 .10^{5}$ & $0.9 .10^{6}$ \\
\hline$B^{+} \rightarrow J / \psi \mathbf{K}^{+}$ & 1700 & 17000 \\
\hline$B^{0} \rightarrow J / \psi \mathbf{K}^{0^{*}}$ & 870 & 8700 \\
\hline
\end{tabular}




\section{B-physics with 100 pb-1 measurements in control channels at $14 \mathrm{TeV}$}

Sensitive tests of understanding of detector properties with strong impact on selected B-physics measurements: masses, lifetimes

\begin{tabular}{|l|l|l|l|l|}
\hline & & $\begin{array}{l}\text { Statitics } \\
\mathbf{1 0 0} \mathbf{p b}-\mathbf{1}\end{array}$ & $\begin{array}{l}\text { Statistical error on } \\
\text { Lifetime }\end{array}$ & $\begin{array}{l}\text { World today } \\
\text { (stat + syst) }\end{array}$ \\
\hline $\mathrm{B}^{+}$ & $\mathrm{B}^{+} \rightarrow \mathbf{J} / \psi \mathbf{K}^{+}$ & 17000 & $1.5 \%$ & $0.4 \%$ \\
\hline $\mathrm{B}^{0}$ & $\mathrm{~B}^{0} \rightarrow \mathbf{J} / \psi \mathbf{K}^{\mathbf{0}^{*}}$ & 8700 & $2.2 \%$ & $0.5 \%$ \\
\hline $\mathrm{B}_{\mathrm{s}}$ & $\mathbf{B}_{\mathrm{s}} \rightarrow \mathrm{J} / \psi \phi$ & 900 & $6 \%$ & $2 \%$ \\
\hline$\Lambda_{\mathrm{b}}$ & $\Lambda_{\mathrm{b}} \rightarrow \mathrm{J} / \psi \Lambda$ & 260 & $8 \%$ & $5 \%$ \\
\hline
\end{tabular}


ATLAS sensitivity in discovery channel

$\mathrm{Br}\left(\mathrm{BO}_{\mathrm{s}} \rightarrow \mu^{+} \mu^{-}\right)$

sensitive to SUSY with $100 \mathrm{pb}-1$ and later

\begin{tabular}{|c|c|c|c|c|}
\hline $\begin{array}{c}\text { Integral } \\
\text { LHC } \\
\text { Luminosity }\end{array}$ & $\begin{array}{c}\text { Signal } \\
\text { ev. after } \\
\text { cuts }\end{array}$ & $\begin{array}{c}\text { BG ev. } \\
\text { after cuts }\end{array}$ & $\begin{array}{c}\text { ATLAS } \\
\text { upper } \\
\text { limit at } \\
90 \% \\
\text { CL }\end{array}$ & $\begin{array}{c}\text { CDF\&D0 } \\
\text { upper } \\
\text { limit at } \\
90 \% \mathrm{CL}\end{array}$ \\
\hline $100 \mathrm{pb}^{-1}$ & $\sim 0$ & $\sim 0.2$ & $6.4 \times 10^{-8}$ & \multirow{3}{*}{$8 \times 10^{-8}$} \\
\hline $10 \mathrm{fb}^{-1}$ & $\sim 7$ & $\sim 20$ & $7.0 \times 10^{-9}$ & \\
\hline $30 \mathrm{fb}^{-1}$ & $\sim 21$ & $\sim 60$ & $6.6 \times 10^{-9}$ & \\
\hline
\end{tabular}


Constraining PDF with early ATLAS data using $W \rightarrow$ IV angular distributions

$$
\begin{aligned}
x_{1,2}=\frac{M}{\sqrt{s}} \exp ( \pm y) \Rightarrow & \text { W production over }|y|<2.5 \text { at } L H C \\
& \text { involves } 10^{-4}<x_{1,2}<0.1 \\
& \Rightarrow \text { region dominated by } g \rightarrow 99
\end{aligned}
$$

Tricoli et al., ATL-PHYS-CONF-2005-008

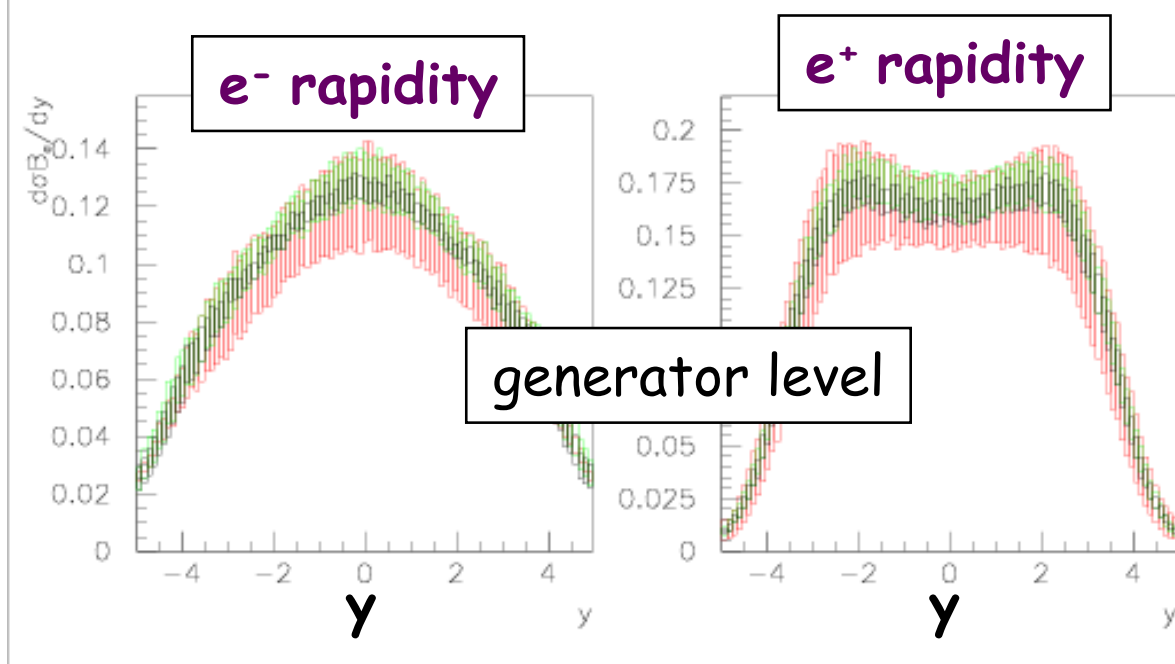

HERWIG +

NLO K-factor

CTEQ61

MRST01

ZEUS-S
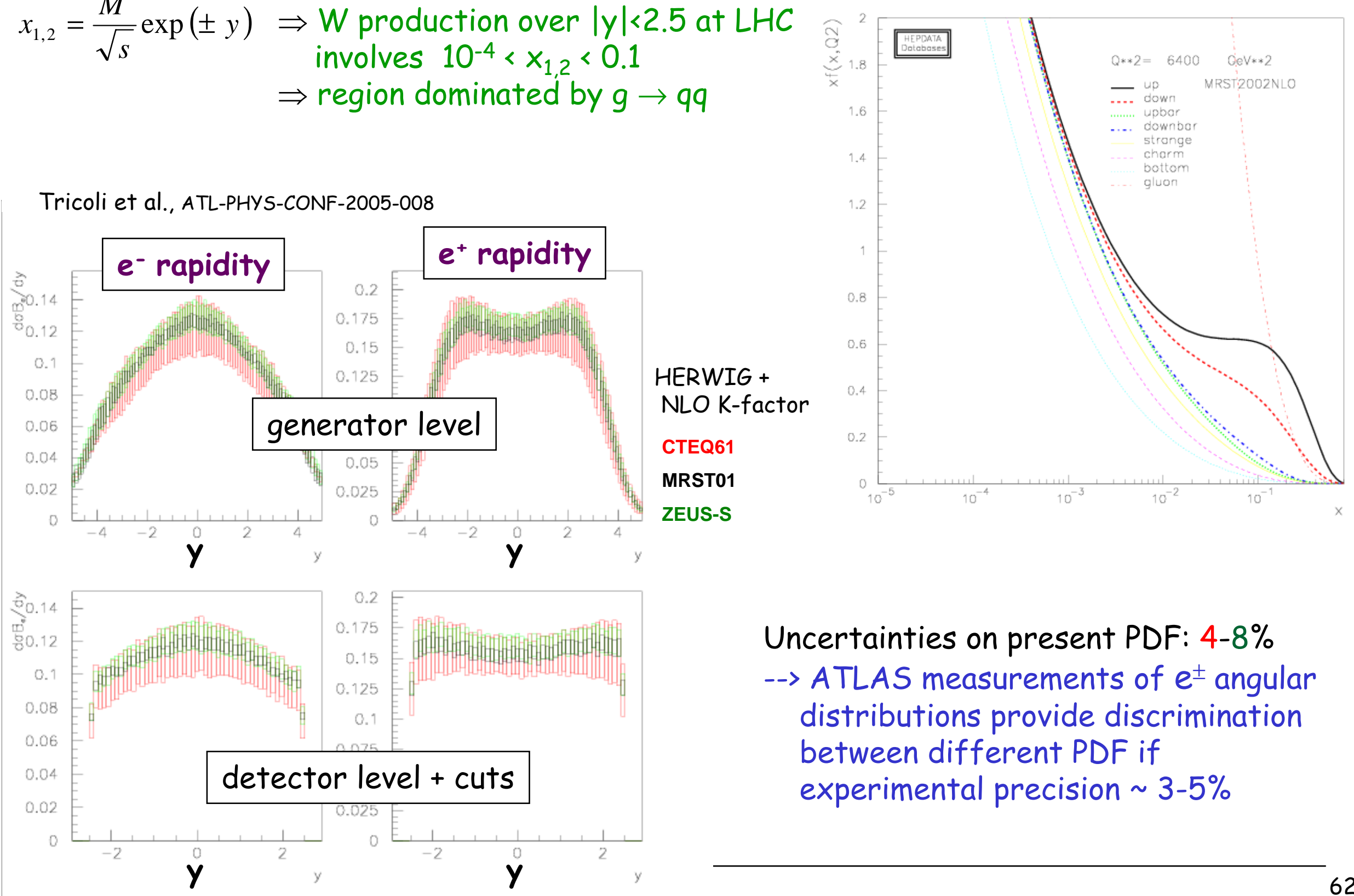

Uncertainties on present PDF: $4-8 \%$

$-\rightarrow$ ATLAS measurements of $e^{ \pm}$angular distributions provide discrimination between different PDF if experimental precision 3-5\% 


\section{Effect of including ATLAS data on PDF fits}

Sample of $10^{6} \mathrm{~W} \rightarrow$ ev generated with CTEQ6.1 and ATLAS fast simulation Statistics corresponds to $~ 100 \mathrm{pb}^{-1}$ $4 \%$ systematic error included by hand (statistical error negligible)
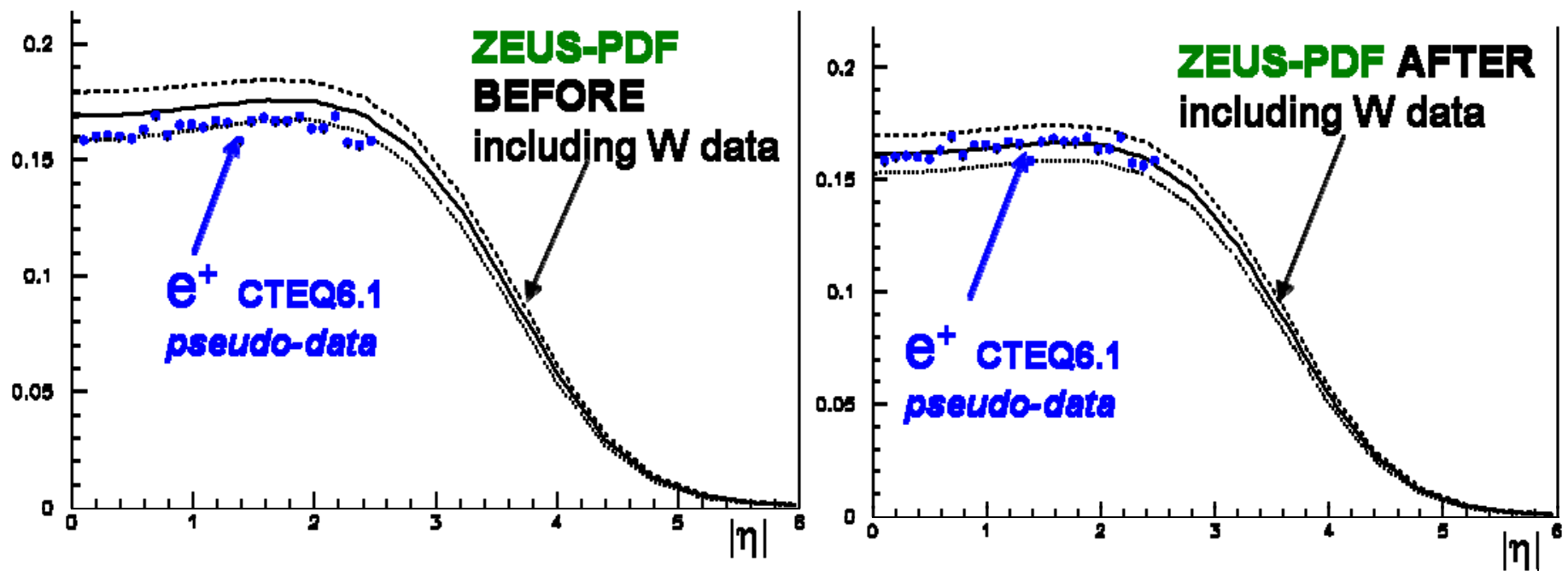

Central value of ZEUS-PDF prediction shifts and uncertainties is reduced Error on low- $x$ gluon shape parameter $\lambda\left(x g(x) \sim x^{-\lambda}\right)$ reduced by $35 \%$

Systematics (e.g. $e^{ \pm}$acceptance vs $\eta$ ) can be controlled to few percent with $Z \rightarrow$ ee $\left(\sim 30000\right.$ events for $\left.100 \mathrm{pb}^{-1}\right)$ 
An "easy case" : Z' of mass 1 TeV with SM-like couplings

\begin{tabular}{|c|c|c|}
\hline \multicolumn{2}{|c}{$Z^{\prime} \rightarrow$ ee, SSM } \\
\hline Mass & $\begin{array}{c}\text { Expected events for } 1 \mathrm{fb}^{-1} \\
\text { (after all cuts) }\end{array}$ & $\begin{array}{c}\int L \mathrm{dt} \text { needed for discovery } \\
\text { (corresponds to 10 observed evts) }\end{array}$ \\
\hline $1 \mathrm{TeV}$ & $\sim 160$ & $\sim 70 \mathrm{pb}^{-1}$ \\
$1.5 \mathrm{TeV}$ & $\sim 30$ & $\sim 300 \mathrm{pb}^{-1}$ \\
$2 \mathrm{TeV}$ & $\sim 7$ & $\sim 1.5 \mathrm{fb}^{-1}$ \\
\hline
\end{tabular}

- large enough signal sample with $\int L d t \sim 100 \mathrm{pb}^{-1}$ up to $m \approx 1 \mathrm{TeV}$ if "reasonable" Z'ee couplings

- dominant Drell-Yan background small

( $<0.2$ events in the region 1400-1600 GeV, $100 \mathrm{pb}^{-1}$ )

- signal as mass peak on top of background

$Z \rightarrow I I+$ jet samples and DY needed for E-calibration and determination of lepton efficiency

F. Gianotti, ICHEP06, Moscow, 02/08/2006

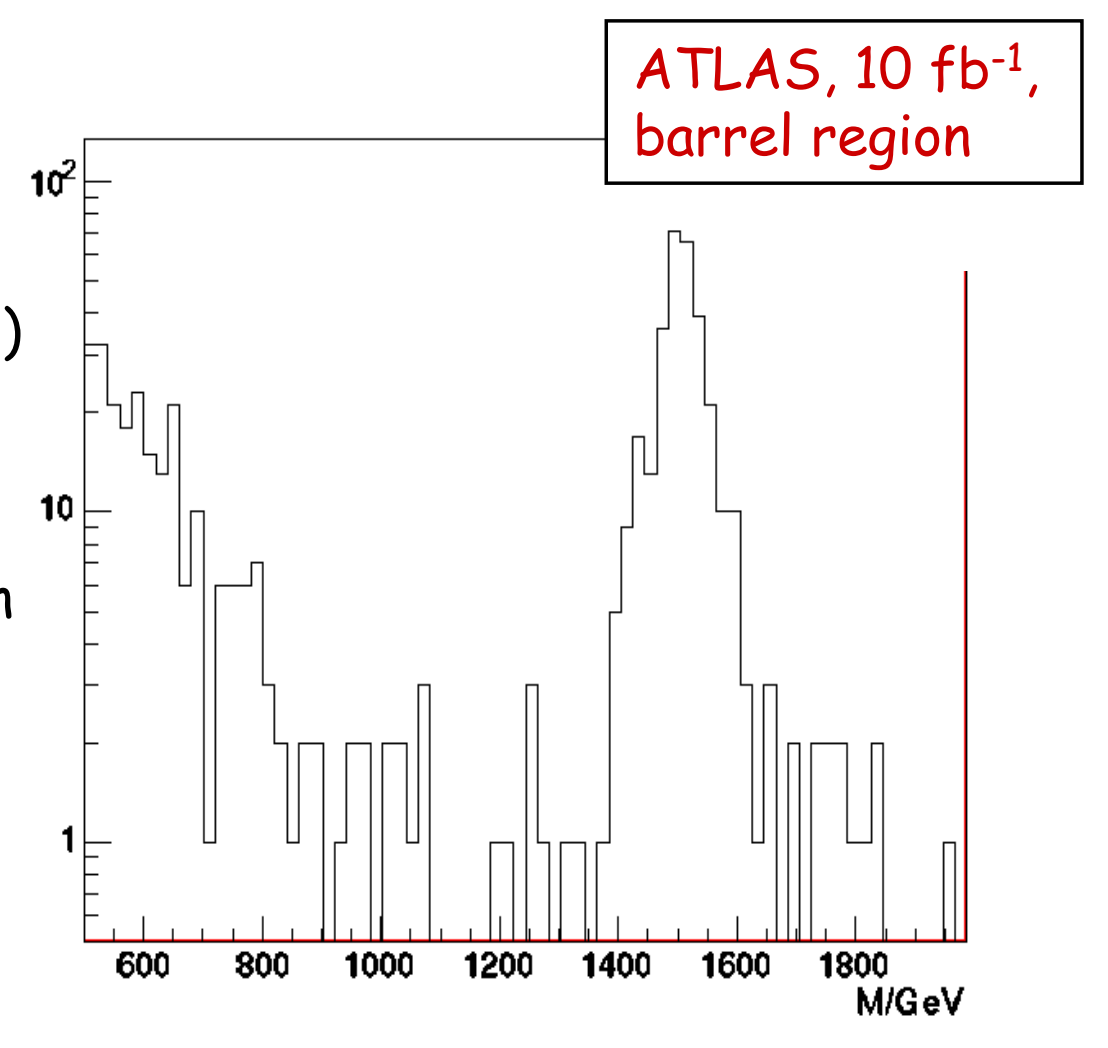




\section{ALPGEN}

QuickTime $^{\mathrm{TM}}$ and a

TIFF (LZW) decompressor

are needed to see this picture. 


\section{SUSY inclusive search}

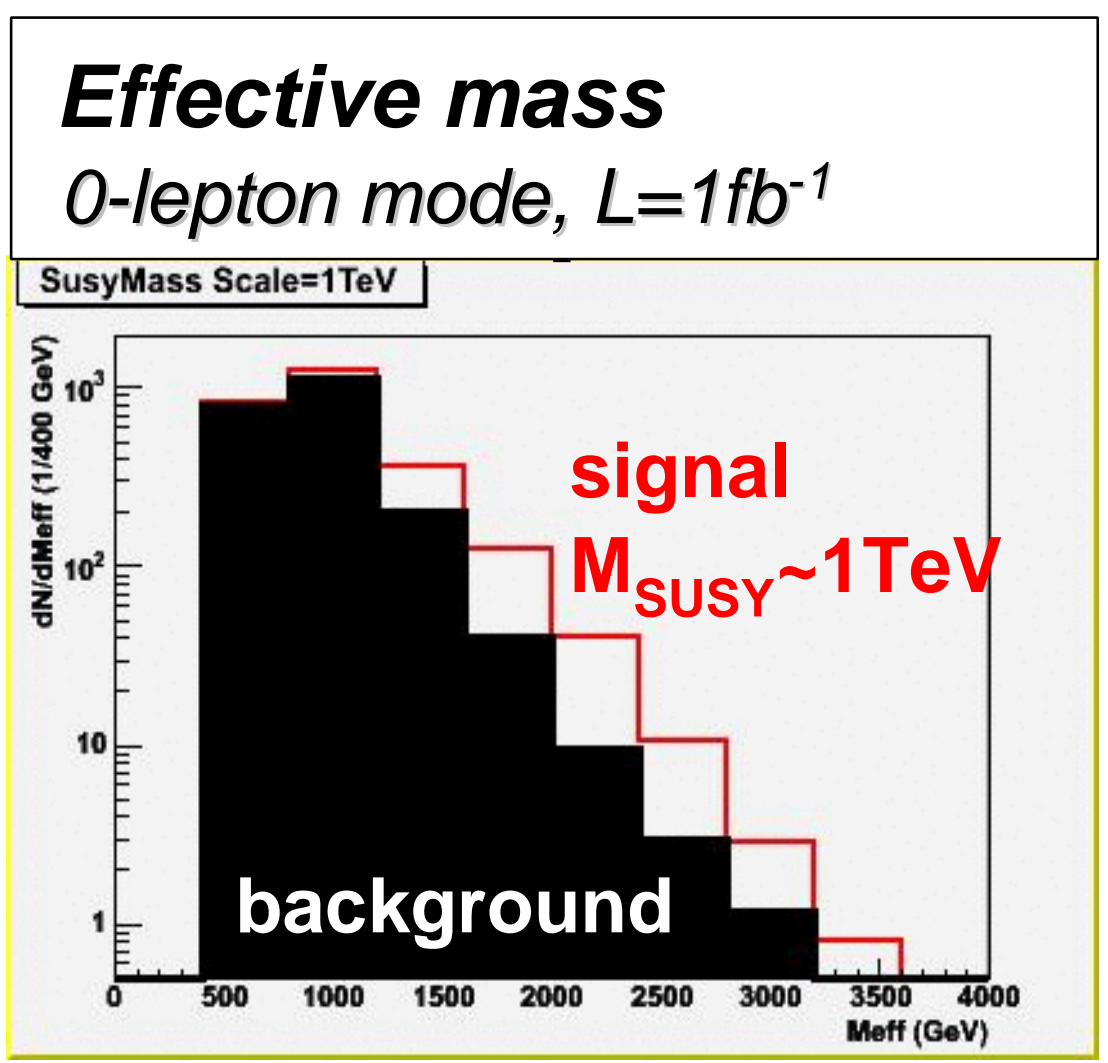

Effective mass (after bkg. subtraction)

0-lepton mode, $L=1 \mathrm{fb}^{-1}$

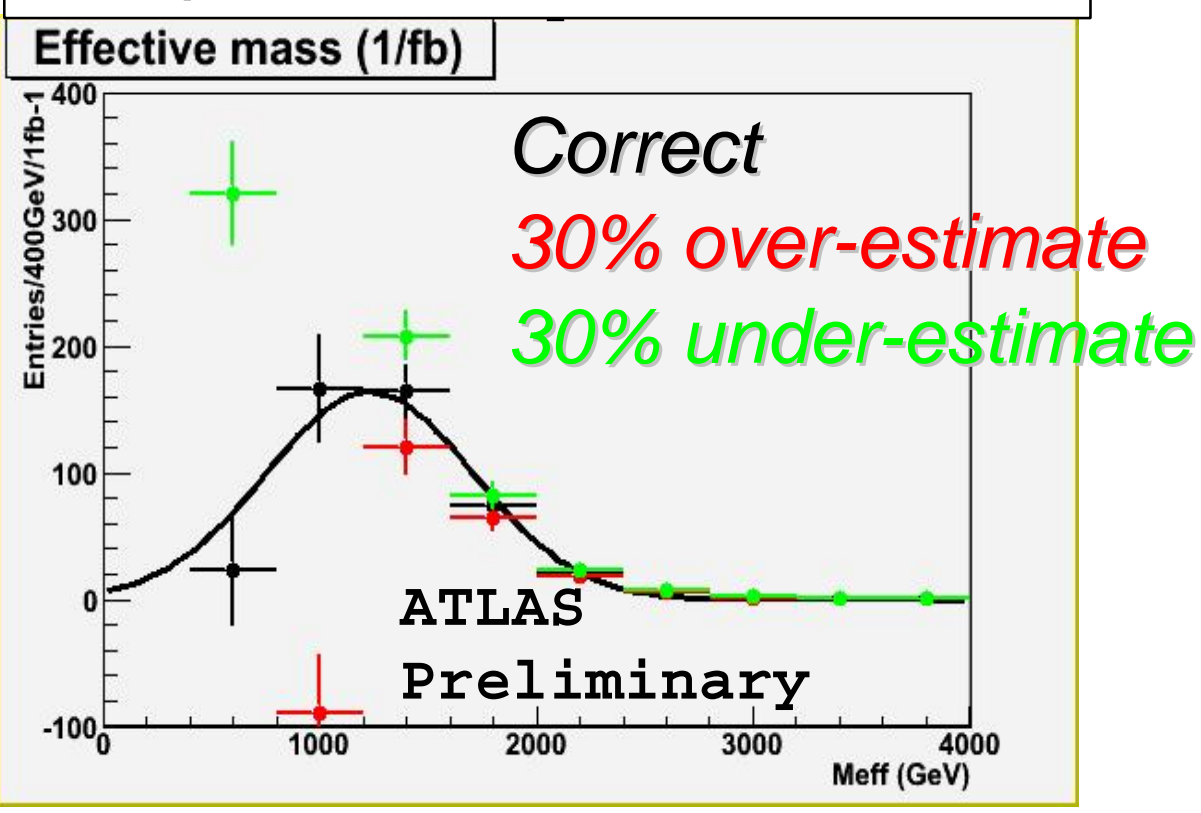

Result with fast simulation.

only scale is changed (slope is same).

Important to understand background scale and slope. 


\section{LHC Kinematic regime}

Kinematic regime for $\mathrm{LHC}$ much broader than currently explored

$\rightarrow$ Test of QCD:

$\square$ Test DGLAP evolution at small $\mathrm{x}$ :

$\square$ Is NLO DGLAP evolution sufficient at so small $x$ ?

$\square$ Are higher orders $\sim \alpha_{s}^{n} \log { }^{m} x$ important?

$\square$ Improve information of high $x$ gluon distribution

At TeV scale New Physics cross section predictions are dominated by high-x gluon uncertainty (not sufficiently well constrained by PDF fits)

At the EW scale theoretical predictions for LHC are dominated by low- $\boldsymbol{x}$ gluon uncertainty (i.e. $W$ and $Z$ masses) $=>$ see later slides

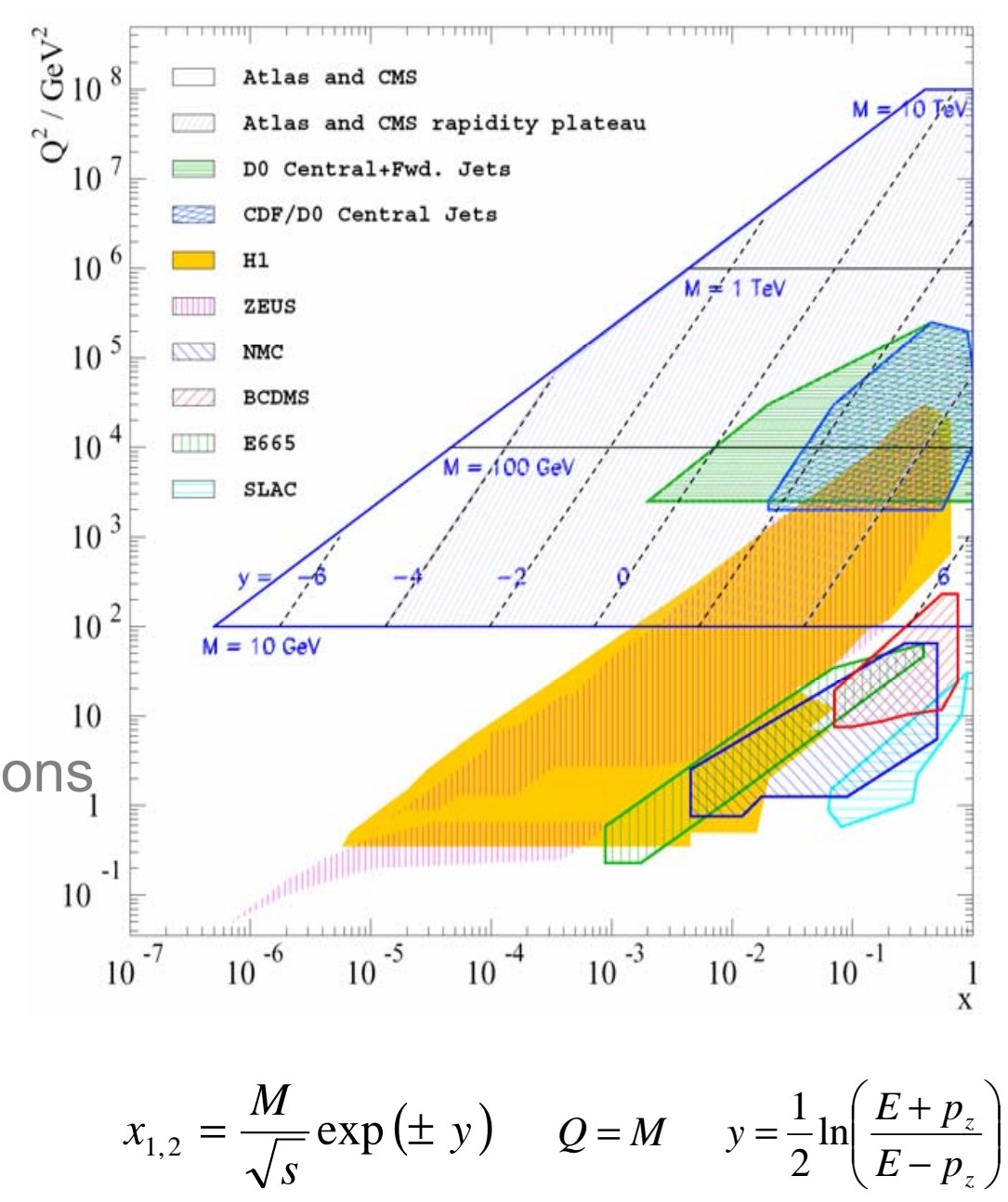

How can we constrain PDF's at LHC?

F. Gianotti, ICHEP06, Moscow, 02/08/2006 


\section{PDF scenario at LHC start up (2007) might be different}

-In most of the relevant $\mathrm{x}$ regions

accessible at LHC

HERA data are most important source of information in PDF determinations

(low-X sea and gluon PDFs)

HERA-II projection shows significant improvement to high-X PDF uncertainties

$\Rightarrow$ relevant for high-scale physics at the LHC

$\rightarrow$ where we expect new physics !!

- significant improvement to valence-quark uncertainties over all-x

- significant improvement to sea and gluon uncertainties at mid-to-high- $x$

- little visible improvement to sea and gluon uncertainties at low-X
-HERA now in second stage of operation (HERA-II)

- substantial increase in luminosity

- possibilities for new measurements

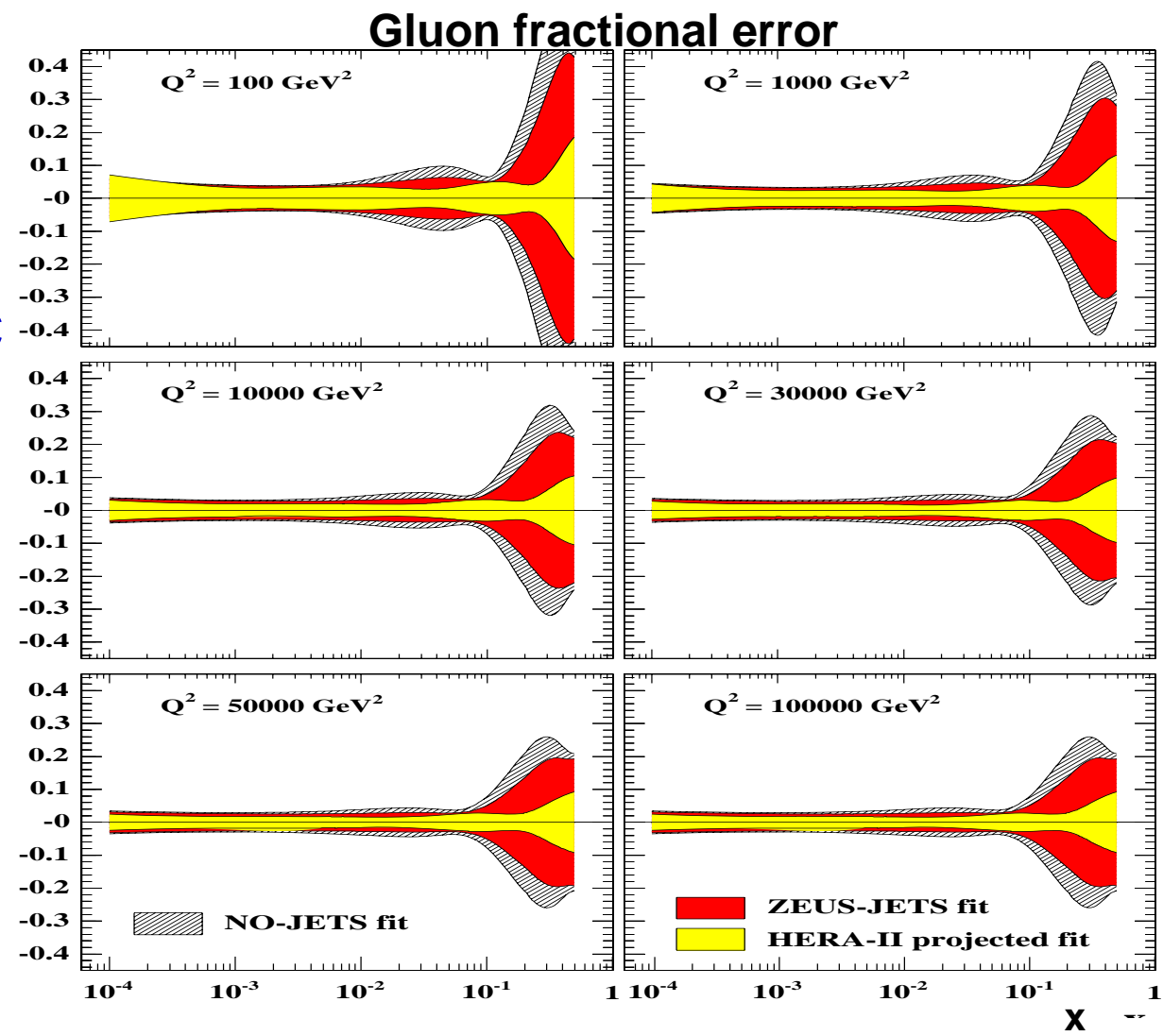

F. Gianotti, ICHEP06, Moscow, 02/08/2006 

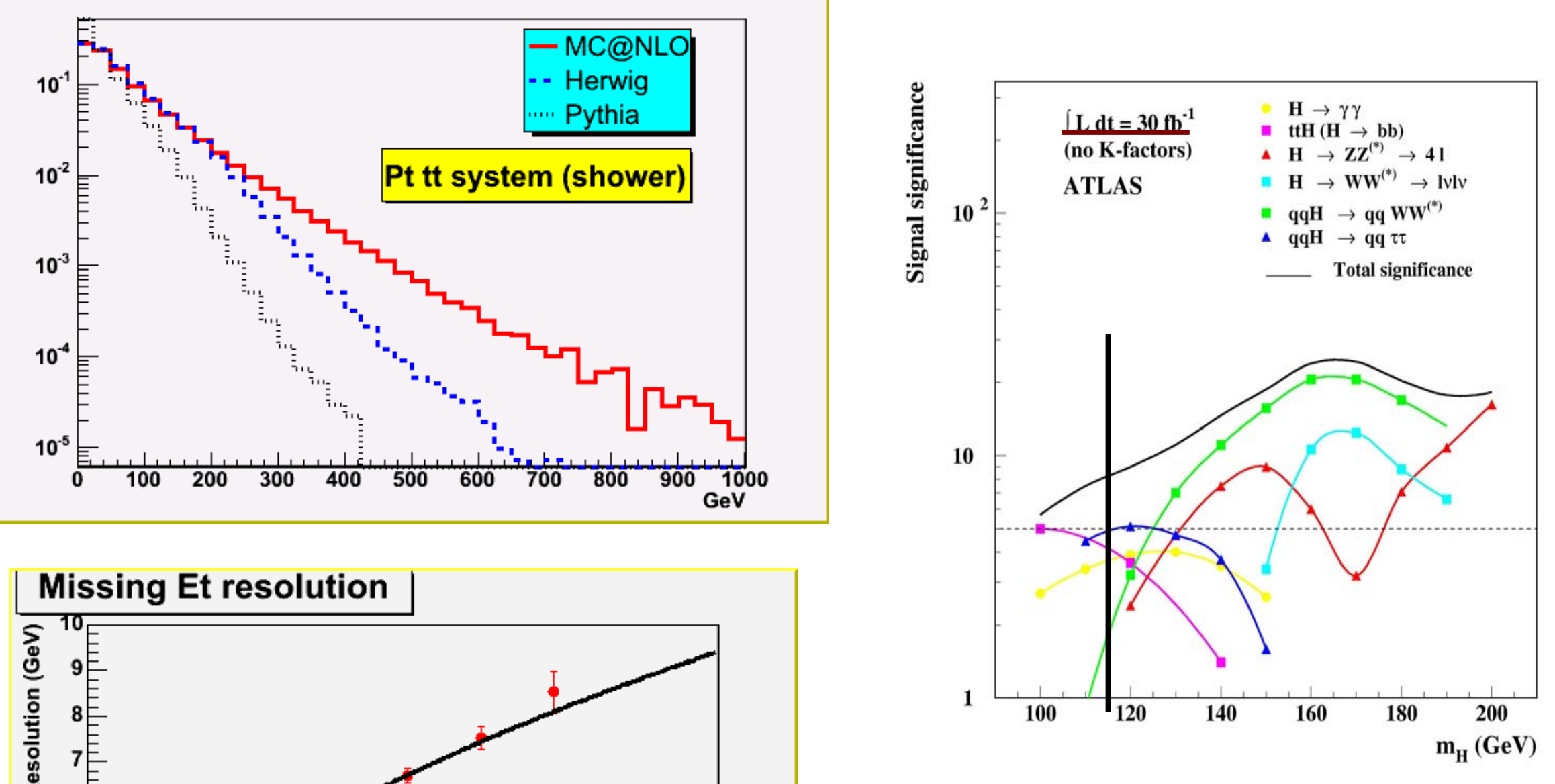

Missing ET resolution can be studied in 2007 (plot made with $35 \mathrm{k}$ minimum-bias events)

F. Gianotti, ICHEP06, Moscow, 02/08/2006 


\begin{tabular}{|c|c|c|c|c|c|}
\hline $\begin{array}{l}\text { Selections (examples ...) } \\
\text { Real thresholds set for } \\
95 \% \text { efficiency at these } E_{T}\end{array}$ & $\begin{array}{l}\text { LVL1 rate }(\mathrm{kHz}) \\
L=1 \times 10^{33} \\
\text { no deferrals }\end{array}$ & \multicolumn{2}{|c|}{$\begin{array}{l}\text { LVL1 rate }(\mathrm{kHz}) \\
L=2 \times 10^{33} \\
\text { no deferrals }\end{array}$} & \multicolumn{2}{|c|}{$\begin{array}{c}\text { LVL1 rate }(\mathrm{kHz}) \\
L=2 \times 10^{33} \\
\text { with deferrals } \\
\text { An example for illustration }\end{array}$} \\
\hline $\begin{array}{l}\text { MU6,8,20 } \\
\text { 2MU6 } \\
\text { EM20i,25,25 } \\
\text { 2EM15i,15,15 } \\
\text { J180,200,200 } \\
3 \mathrm{~J} 75,90,90 \\
\text { 4J55,65,65 } \\
\text { J50+xE50,60,60 } \\
\text { TAU20,25,25 + xE30 } \\
\text { MU10+EM15i } \\
\text { Others (pre-scaled, etc.) }\end{array}$ & $\begin{array}{c}23 \\
--- \\
11 \\
2 \\
0.2 \\
0.2 \\
0.2 \\
0.4 \\
2 \\
--- \\
5\end{array}$ & $\begin{array}{l}\longrightarrow \\
\longrightarrow\end{array}$ & $\begin{array}{l}19 \\
0.2 \\
12 \\
4 \\
0.2 \\
0.2 \\
0.2 \\
0.4 \\
2 \\
0.1 \\
5\end{array}$ & $\longrightarrow$ & $\begin{array}{l}0.8 \\
0.2 \\
12 \\
4 \\
0.2 \\
0.2 \\
0.2 \\
0.4 \\
2 \\
0.1 \\
5\end{array}$ \\
\hline Total & $\sim 44$ & & $\sim 43$ & & $\sim 25$ \\
\hline & $\begin{array}{l}\text { LVL1 designed for } 75 \\
\rightarrow \text { room for factor } \sim\end{array}$ & & & $\begin{array}{l}\text { ely max } \\
\text { room } f\end{array}$ & $\begin{array}{l}\text { ordable rate, } \\
\text { fety factor }\end{array}$ \\
\hline
\end{tabular}




\section{(3) Which data samples?}

High-Level-Trigger output

Total trigger rate to storage at $2 \times 10^{33}$ reduced from $~ 540 \mathrm{~Hz}$ (HLT/DAQ TP, 2000) to $\sim 200 \mathrm{~Hz}$ (now)

$\downarrow$

\begin{tabular}{|c|c|c|}
\hline Selection (examples ... & Rate to storage at $2 \times 10^{33}(\mathrm{~Hz})$ & Physics motivations (examplle \\
\hline e25i, 2e15i & $\sim 40(55 \% \mathrm{~W} / \mathrm{b} / \mathrm{c} \rightarrow \mathrm{eX})$ & Low-mass Higgs $\left(t+H, H \rightarrow 4 \ell,{ }^{\prime}\right.$ \\
\hline$\mu 20 i, 2 \mu 10$ & $\sim 40(85 \% \mathrm{~W} / \mathrm{b} / \mathrm{c} \rightarrow \mu \mathrm{X})$ & W, Z, top, New Physics? \\
\hline $160 i, 2,20 i$ & - 40 (57\% prompty) & $H \rightarrow n$, New Physies \\
\hline$j 400,3 j 165,4 j 110$ & $\sim 25$ & $\begin{array}{l}\text { (e.g. } X \rightarrow \text { ryy } m_{X} \sim 500 \mathrm{GeV} \\
\text { Overlap with Tevatron for } n \in \text { u }\end{array}$ \\
\hline & & $X \rightarrow \mathrm{j} j$ in danger ... \\
\hline$j 70+x E 70$ & $\sim 20$ & SUSY: $400 \mathrm{GeV}$ squarks/gl \\
\hline$\tau 35+x E 45$ & $\sim 5$ & MSSM Higgs, New Physics \\
\hline $2 \mu 6\left(+m_{B}\right)$ & $\sim 10$ & $\begin{array}{l}\text { (3rd family !) ? More difficul } \\
\text { Rare decays } B \rightarrow \mu \mu X\end{array}$ \\
\hline $\begin{array}{l}\text { Others } \\
\text { (pre-scaled, exclusive, }\end{array}$ & -20 & Onty $10 \%$ of total! \\
\hline Total & $\sim 200$ & $\begin{array}{l}\text { No safety factor included. } \\
\text { "Signal" }(W, \gamma, \text { etc. }): \sim 100\end{array}$ \\
\hline & & \\
\hline
\end{tabular}

Best use of spare capacity when $L<2 \times 10^{33}$ being investigated 


\section{ALICE Experimental Layout}

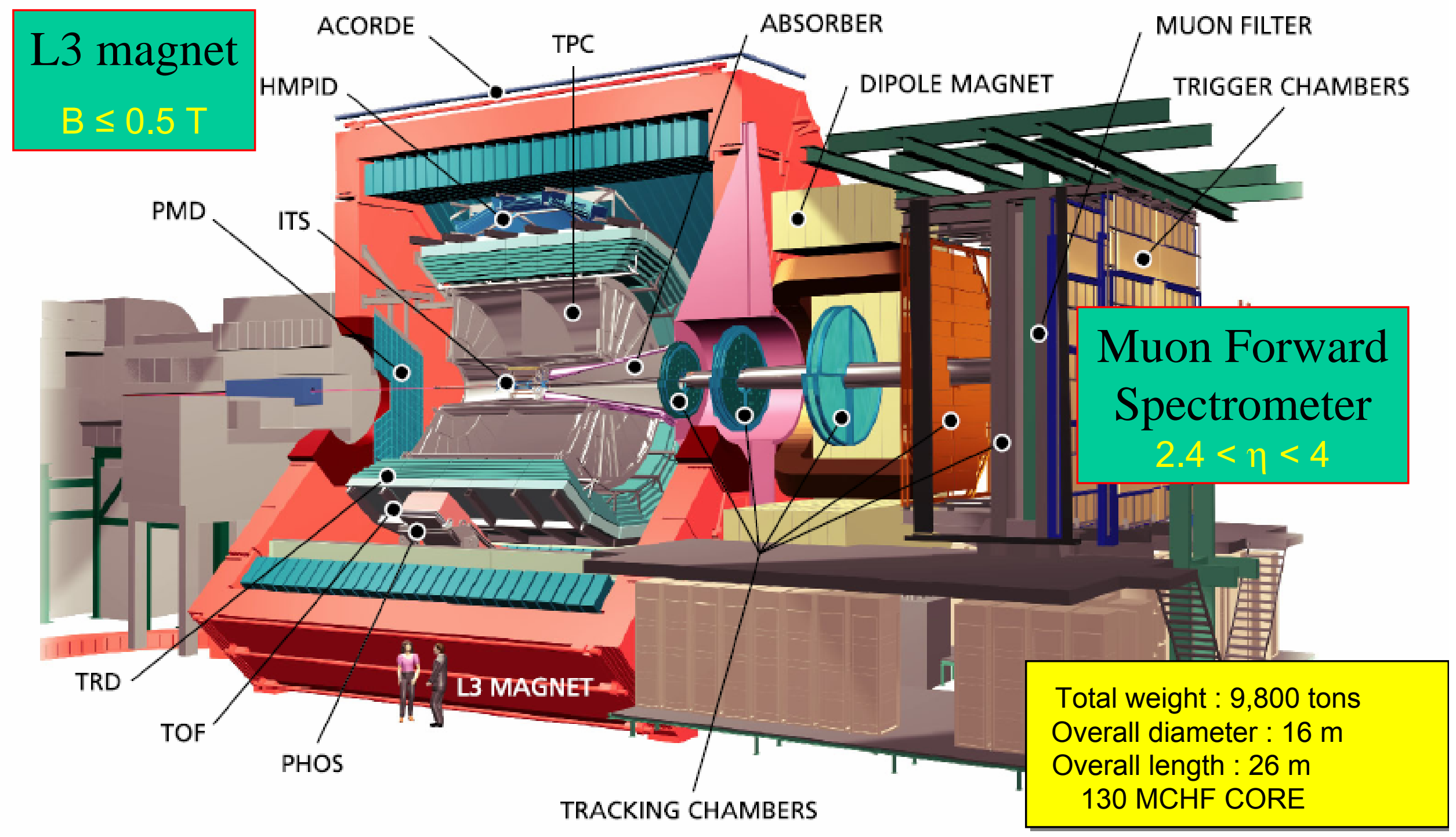

F. Gianotti, ICHEPO6, Moscow, 02/08/2006 


\section{MUON spectrometer set-up}

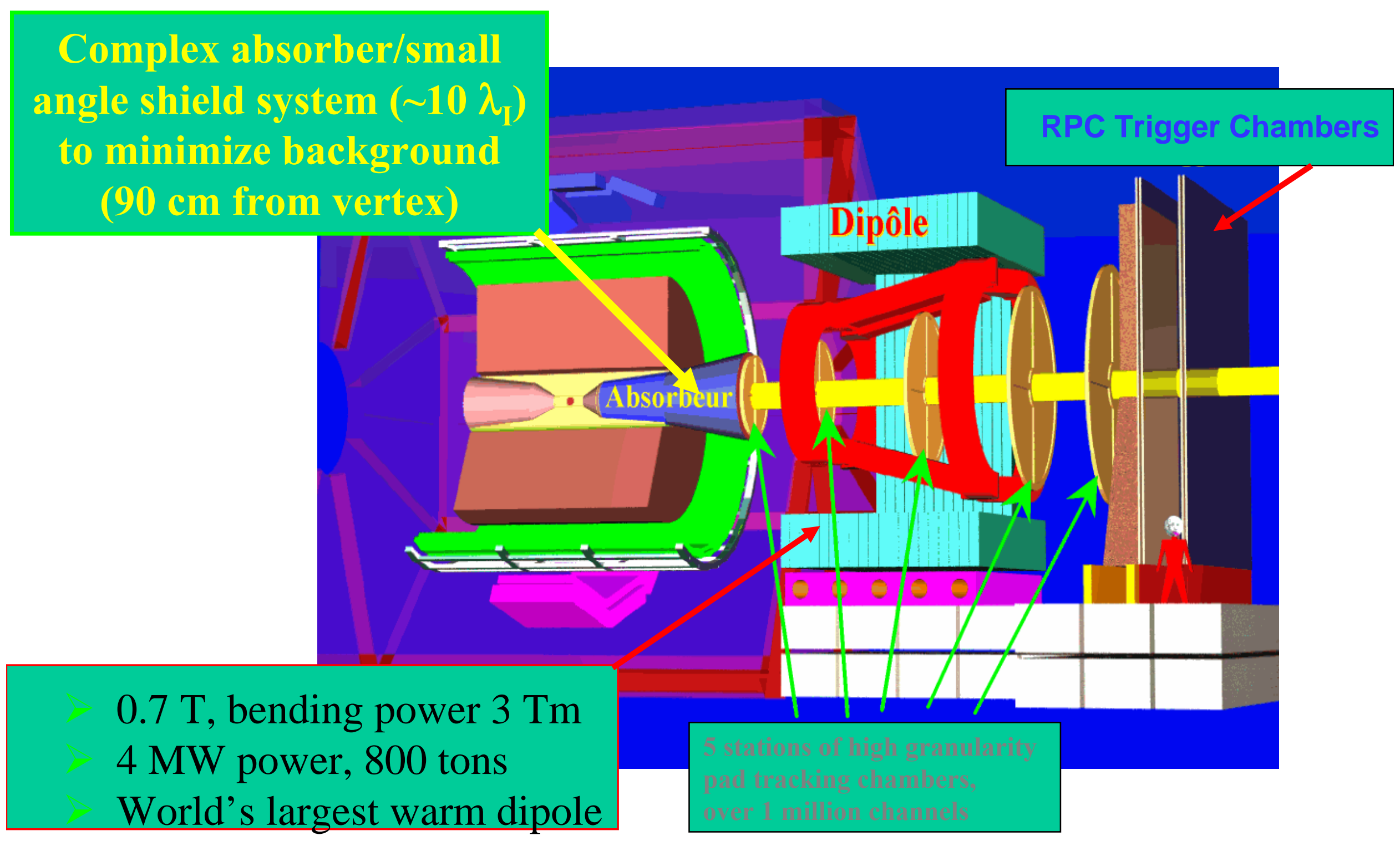




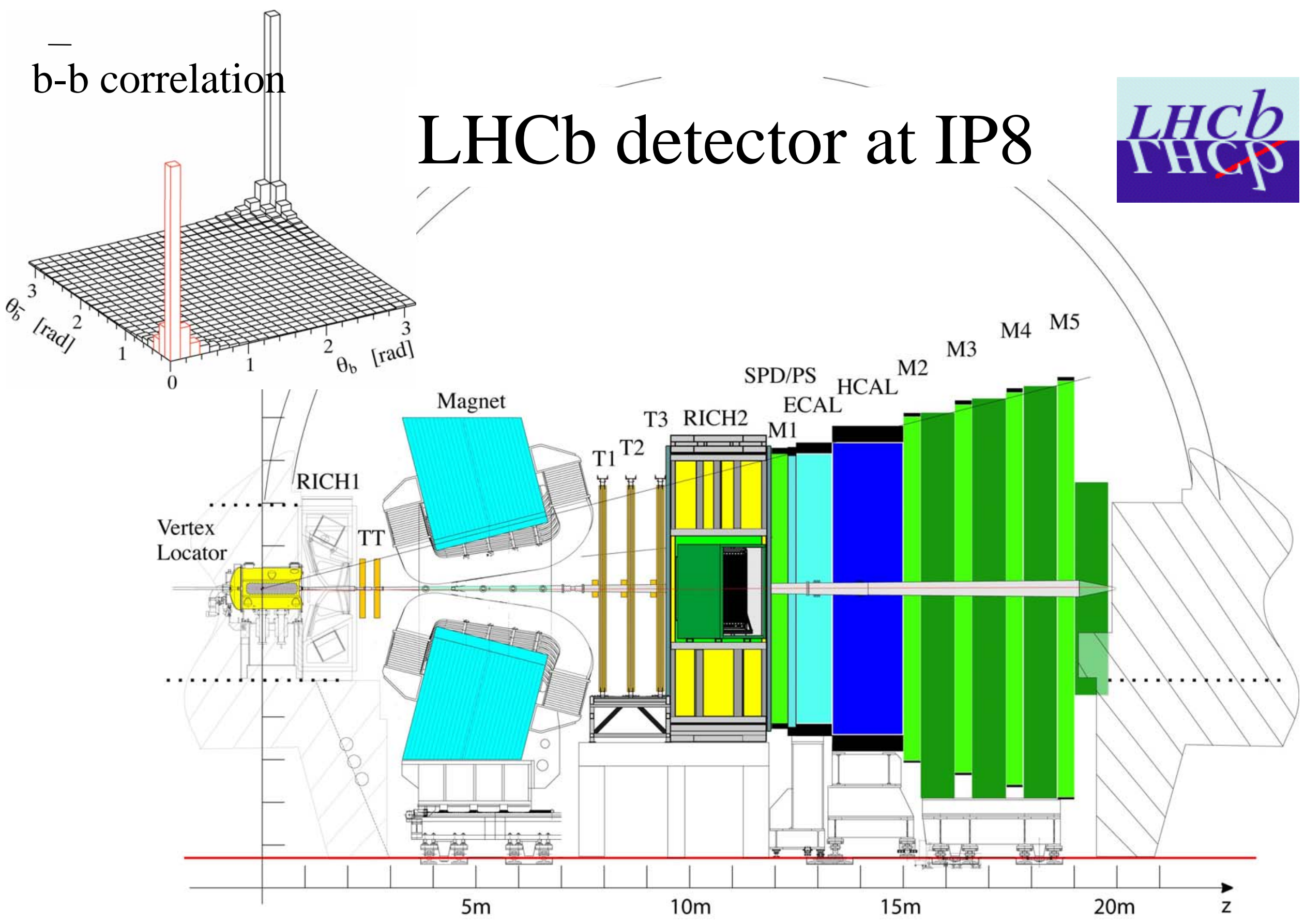

F. Gianotti, ICHEP06, Moscow, 02/08/2006 


\section{Compact Muon Solenoid (CMS)}

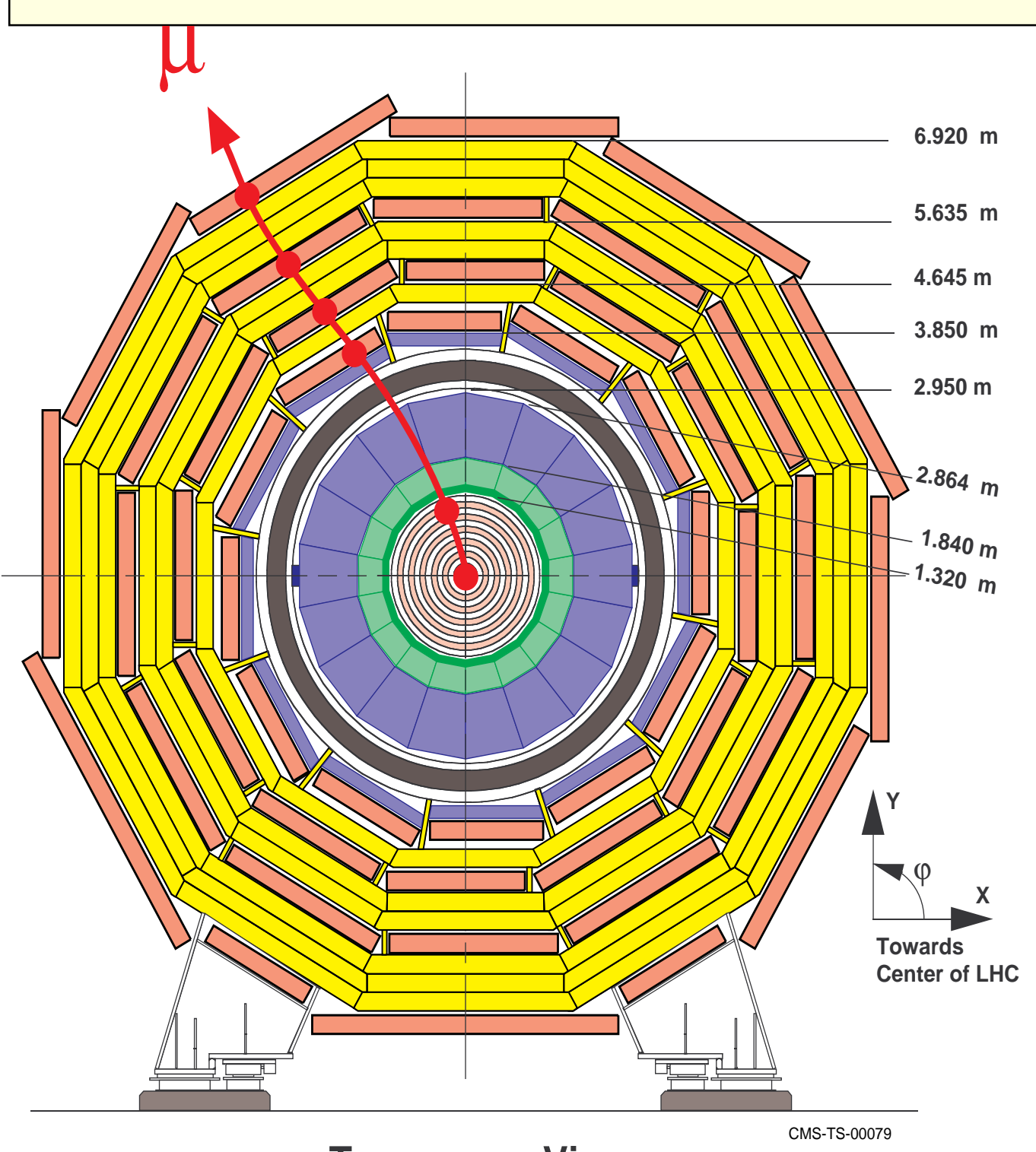

Strong Field 4T

Compact design

Solenoid for Muon $\mathrm{P}_{\mathrm{t}}$ trigger in transverse plane

Redundancy: 4 muon stations with $32 \mathrm{r}$-phi measurements

$\Delta \mathrm{P}_{\mathrm{t}} / \mathrm{P}_{\mathrm{t}} \sim 5 \% @ 1 \mathrm{TeV}$ for reasonable space resolution of muon chambers $(200 \mu \mathrm{m})$

Transverse View

F. Gianotti, ICHEPO6, Moscow, 02/08/2006 


\section{Muon Reconstruction (Momentum Res.)}

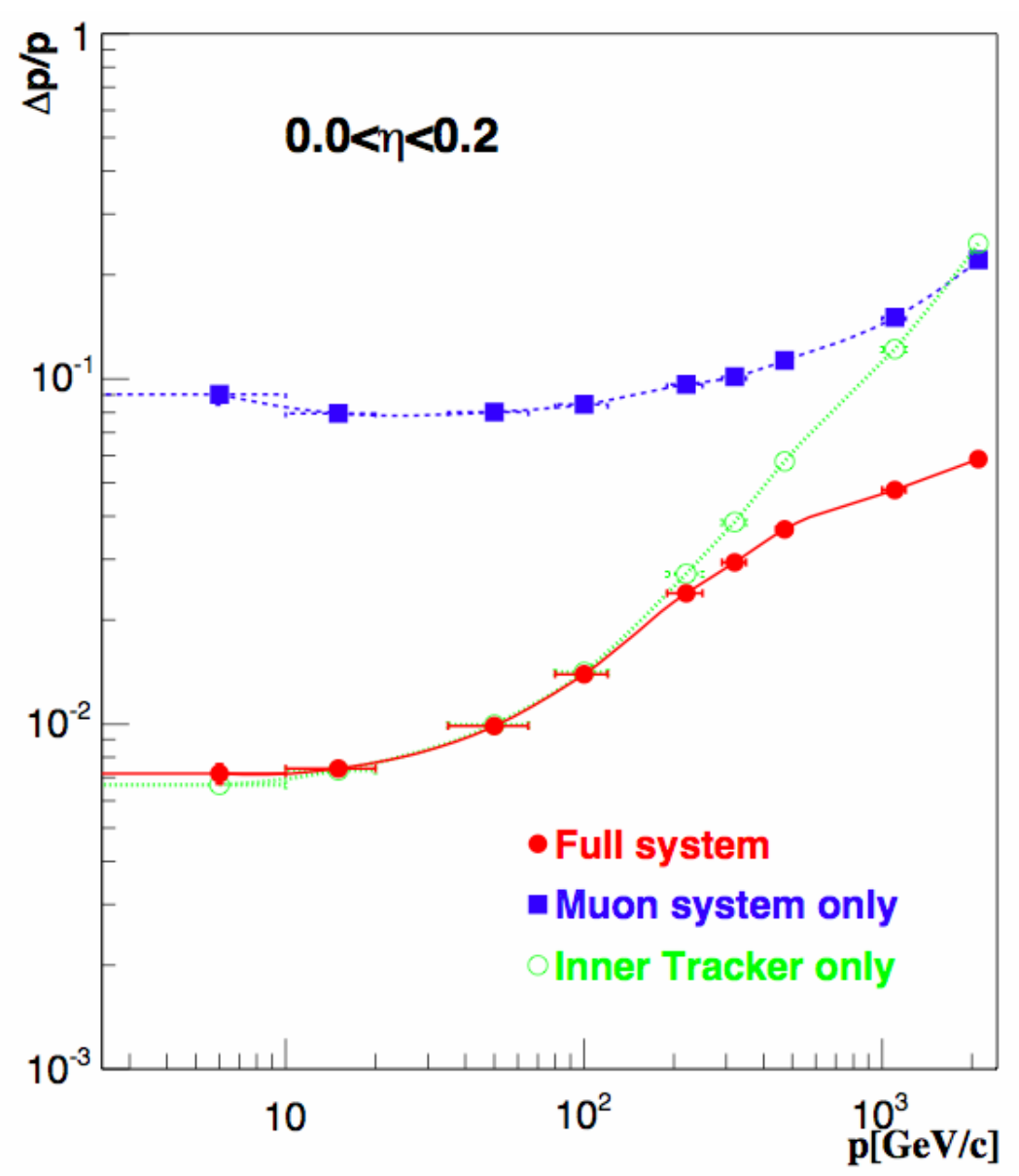

CMS 


\section{Muon System}

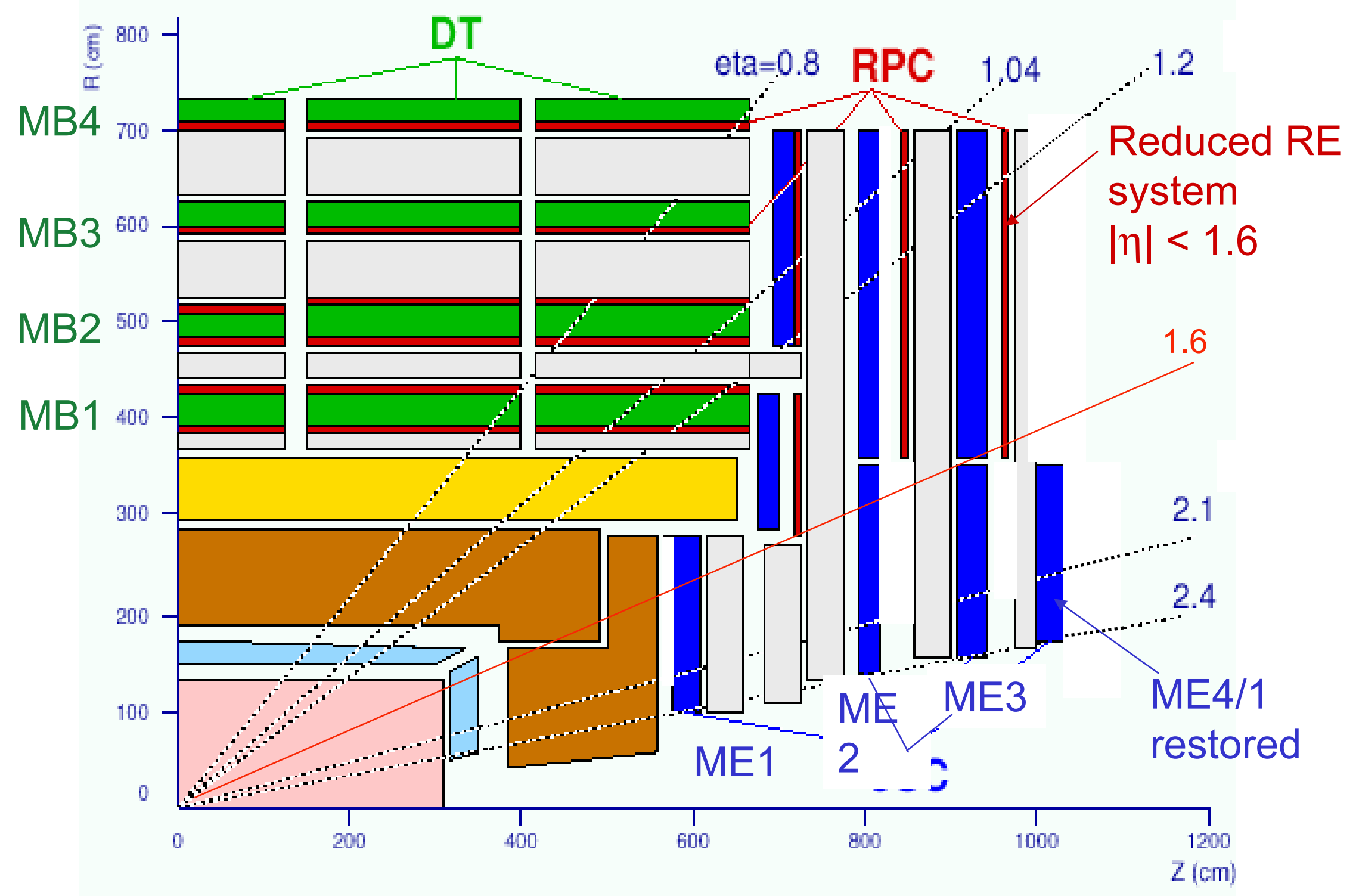

F. Gianotti, ICHEP06, Moscow, 02/08/2006 


\section{ECAL: PbWO4 Crystals}

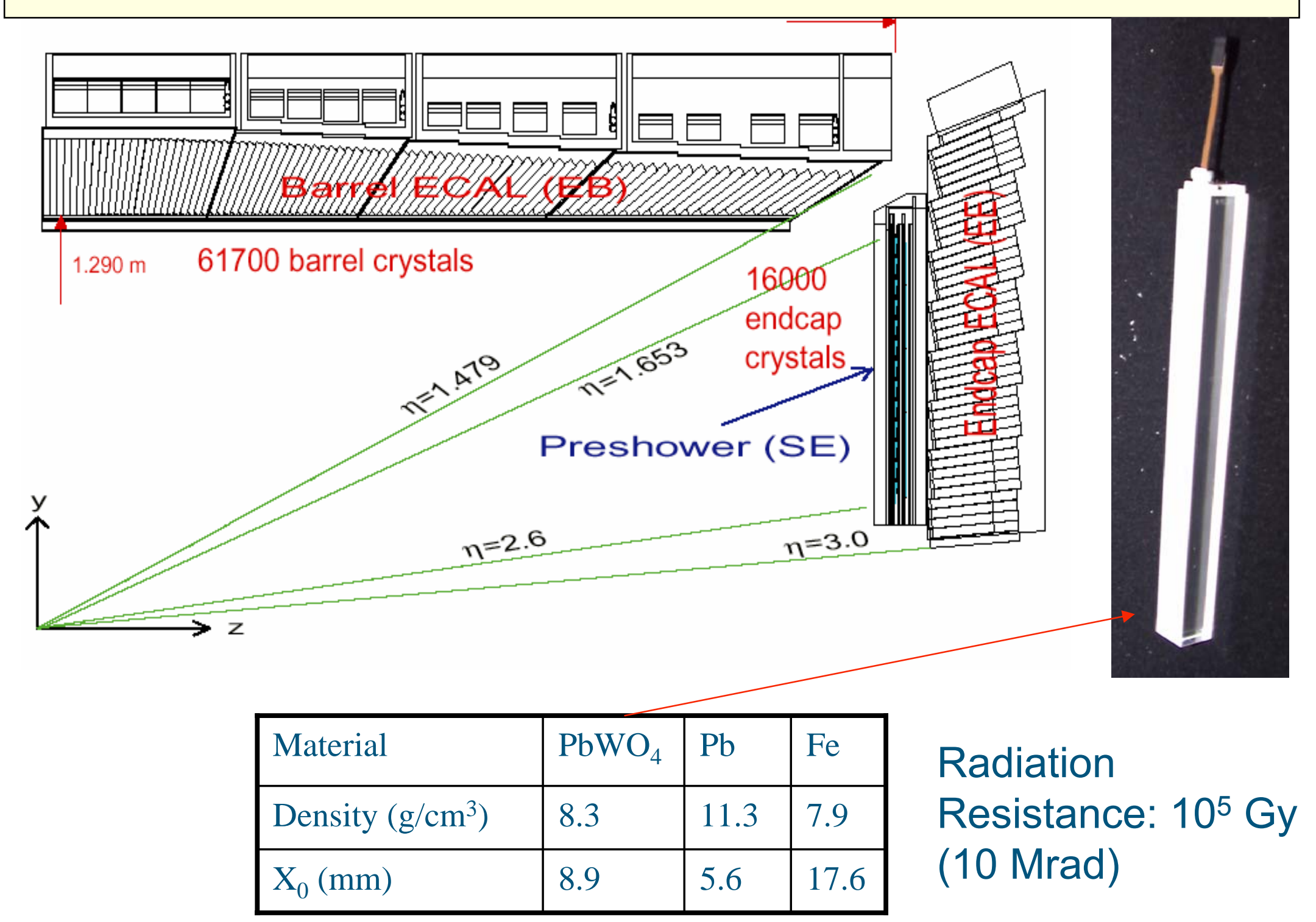

F. Gianotti, ICHEP06, Moscow, 02/08/2006 


\section{Inner Tracker}

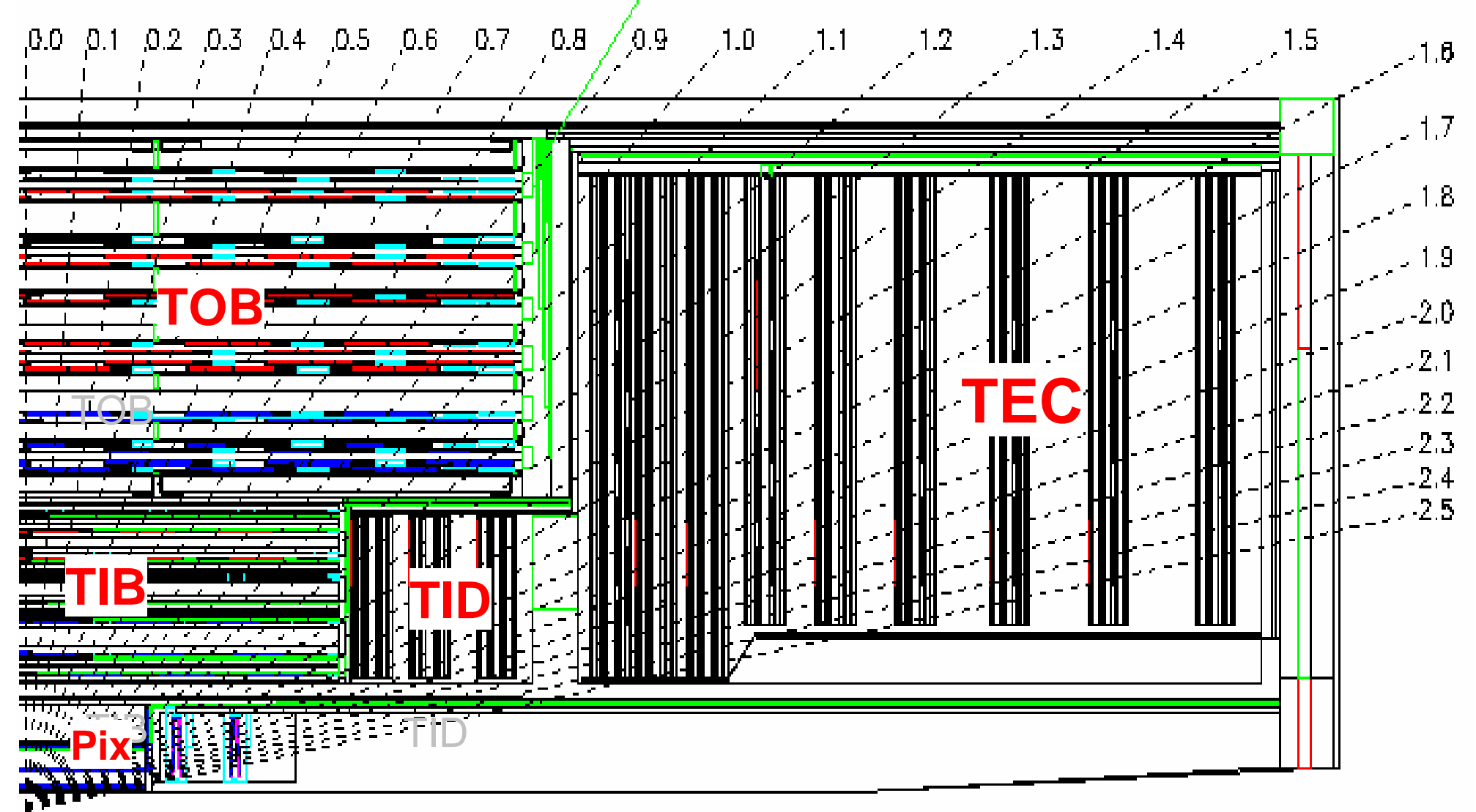

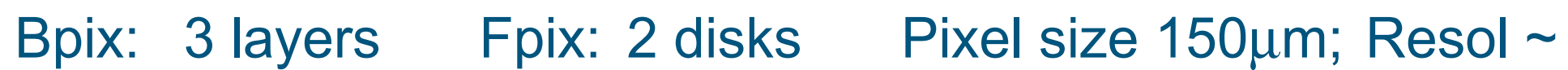
$20 \mu \mathrm{m}$

TIB: 4 layers TID: 3 disks Pitch: $80 \mu \mathrm{m}$ to $200 \mu \mathrm{m}$ TOB: 6 layers TEC: 9 disks Resol: $20 \mu \mathrm{m}$ to $50 \mu \mathrm{m}$ 


\section{Laser Alignment System}

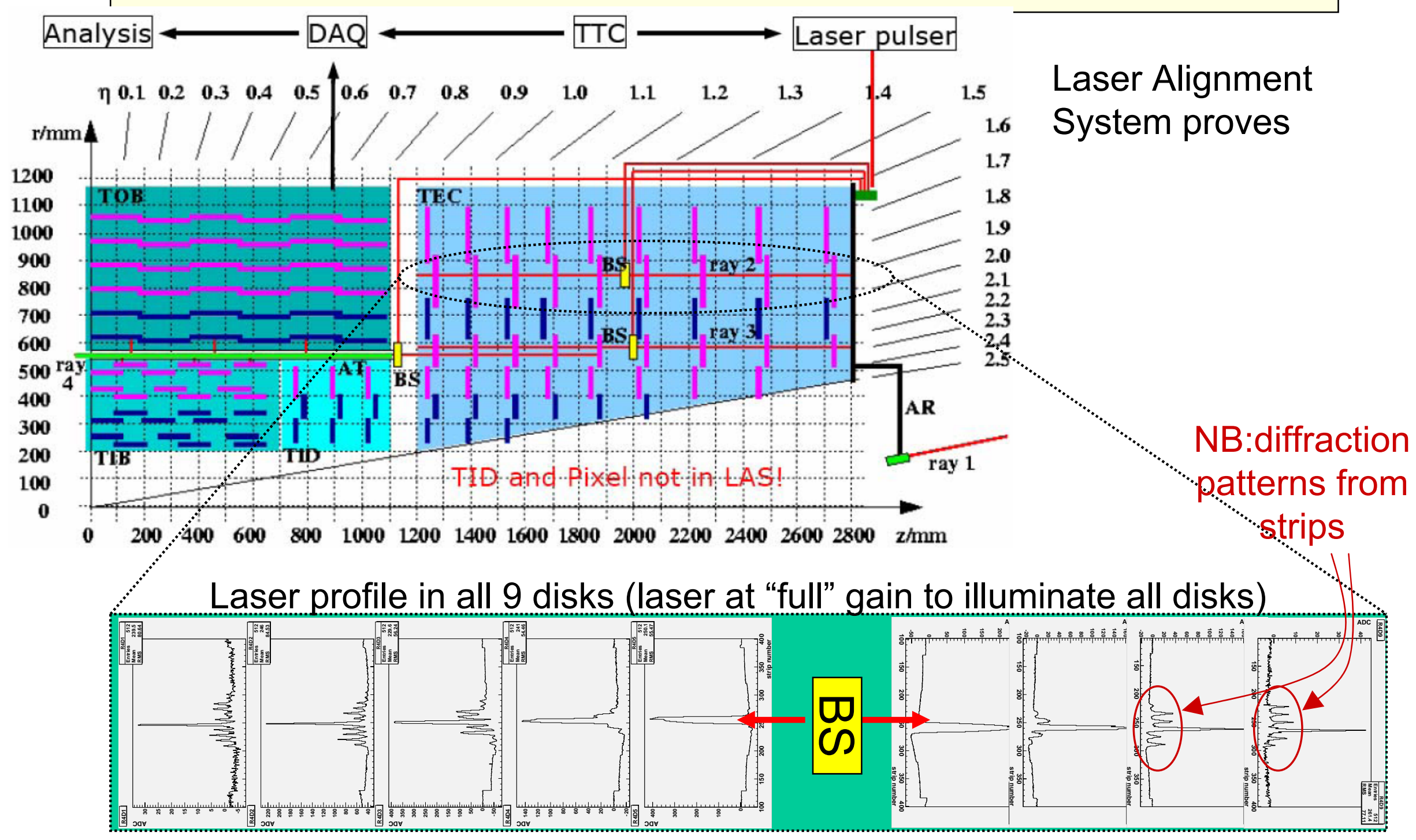

F. Gianotti, ICHEP06, Moscow, 02/08/2006 


\section{Hadronic Calorimeter: HCAL}

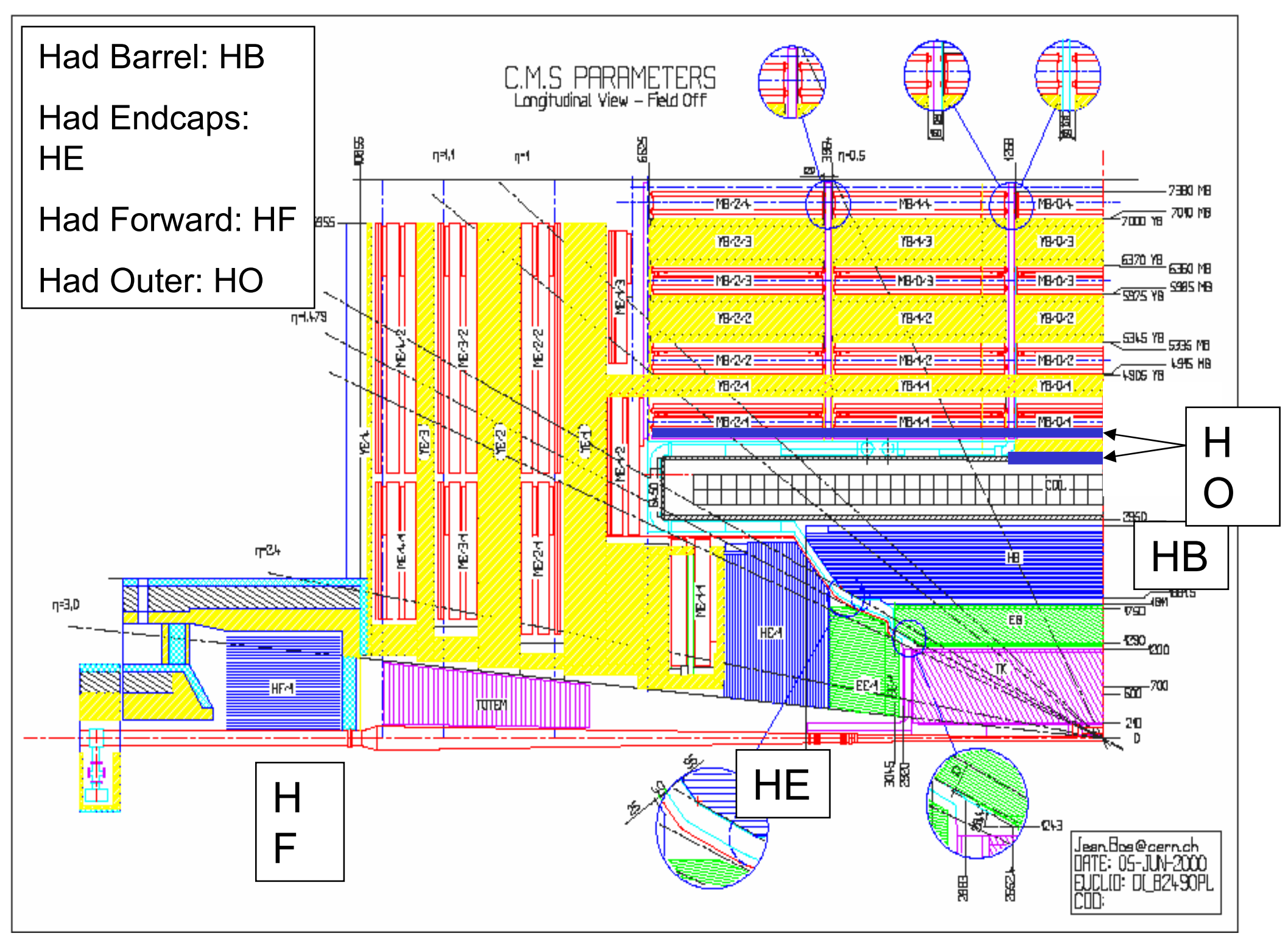

F. Gianotti, ICHEPO6, Moscow, 02/08/2006 


\section{The low mass cable problem}

- The barrel Al cables are made of $100 \mu \mathrm{m}$ wire for signal and $300 \mu \mathrm{m}$ for power (21 wires in a bundle), both wire-bonded on a small printed board + mini connector $(\sim 1 \mathrm{~m}$, module $\rightarrow$ PP0).

- The initial interpretation was that the cable was stressed during manipulation and strain relief and better practice should solve the problem.

- Visual inspection and electrical tests after stress on all production started and found that $\sim 50 \%$ of the 2000 cable produced are bad or likely to evolve bad.

- Indeed the insulation was found to have cracks and, being the insulator much stronger than a $100 \mu \mathrm{m} \mathrm{Al}$ wire, any stress would concentrate on the wire thus breaking it (sooner or later), this is not true for the thicker power wires.

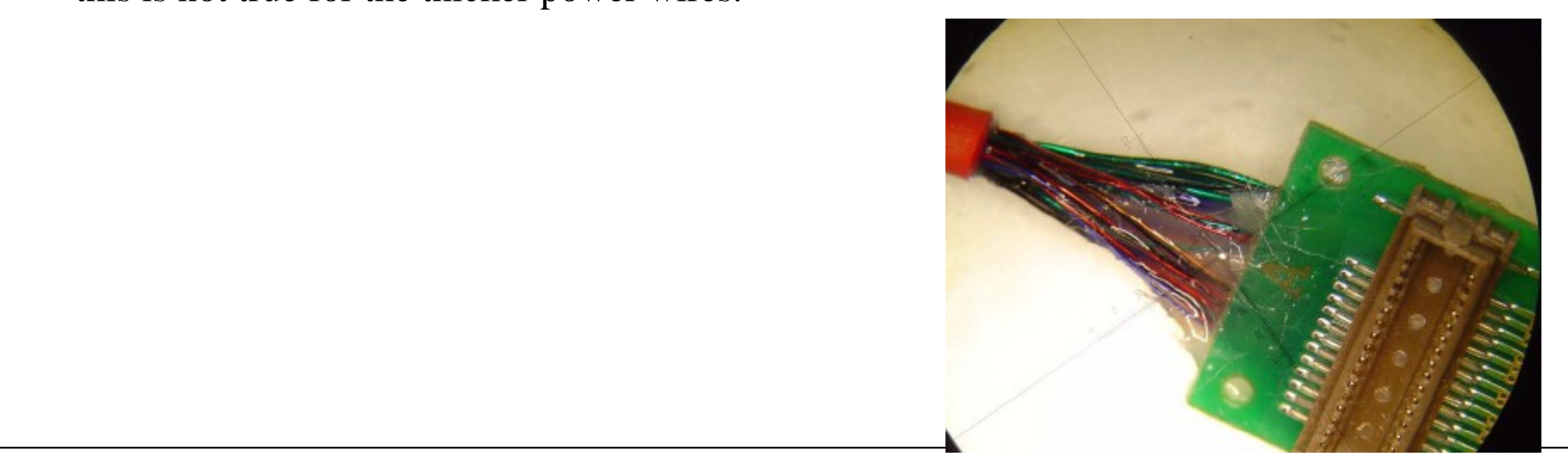

F. Gianotti, ICHEP06, Moscow, 02/08/2006 